\title{
Retrotransposon instability dominates the acquired mutation landscape of mouse induced pluripotent stem cells
}

Patricia Gerdes ${ }^{1,10}$, Sue Mei Lim ${ }^{2,3,4,10}$, Adam D. Ewing ${ }^{1,10}$, Michael R. Larcombe ${ }^{2,3,4}$, Dorothy Chan $^{1}$, Francisco J. Sanchez-Luque ${ }^{1,5}$, Lucinda Walker ${ }^{1}$, Cini James ${ }^{1}$, Anja S. Knaupp ${ }^{2,3,4}$, Patricia E. Carreira ${ }^{1}$, Christian M. Nefzger ${ }^{2,3,4}$, Ryan Lister ${ }^{6,7}$, Sandra R. Richardson ${ }^{1, *}$, Jose M. Polo ${ }^{2,3,4,9, *}$, and Geoffrey J. Faulkner ${ }^{1,8, *}$

${ }^{1}$ Mater Research Institute - University of Queensland, TRI Building, Woolloongabba QLD 4102, Australia.

${ }^{2}$ Department of Anatomy \& Developmental Biology, Monash University, Melbourne VIC 3800, Australia.

${ }^{3}$ Development and Stem Cells Program, Monash Biomedicine Discovery Institute, Melbourne VIC 3800, Australia.

${ }^{4}$ Australian Regenerative Medicine Institute, Monash University, Melbourne VIC 3800, Australia. ${ }^{5}$ GENYO. Pfizer-University of Granada-Andalusian Government Centre for Genomics and Oncological Research, PTS Granada 18016, Spain.

${ }^{6}$ Australian Research Council Centre of Excellence in Plant Energy Biology, School of Molecular Sciences, The University of Western Australia, Perth WA 6009, Australia.

${ }^{7}$ Harry Perkins Institute of Medical Research, Perth WA 6009, Australia.

${ }^{8}$ Queensland Brain Institute, University of Queensland, Brisbane QLD 4072, Australia.

${ }^{9}$ Adelaide Centre for Epigenetics and The South Australian Immunogenomics Cancer Institute, The University of Adelaide, Adelaide SA 5005, Australia.

${ }^{10}$ These authors contributed equally

*Corresponding authors: sandra.richardson@mater.uq.edu.au (S.R.R.); jose.polo@monash.edu (J.M.P.); faulknergj@gmail.com (G.J.F.) 


\section{Abstract}

2 Induced pluripotent stem cells (iPSCs) may differentiate into any cell of the body and as such have revolutionized biomedical research and regenerative medicine. Unlike their human counterparts,

4 mouse iPSCs (miPSCs) are reported to silence transposable elements (TEs) and prevent TE-

5 mediated mutagenesis. Here we applied short- or long-read genome sequencing to 30 bulk miPSC

6 lines reprogrammed from 10 parental cell types, as well as 18 single-cell miPSC clones. While

7 single nucleotide variants and structural variants restricted to miPSCs were rare, we found 55 de

8 novo TE insertions, including examples intronic to Brcal and Dmd. LINE-1 (L1) retrotransposon

9 families were profoundly hypomethylated in miPSCs, beyond other TEs and the genome overall, and harbored alternative promoters for protein-coding genes. Treatment with the L1 reverse

11 transcriptase inhibitor lamivudine did not hinder reprogramming, pointing to a viable strategy to 12 block retrotransposition. These experiments reveal the complete spectrum and potential significance of mutations acquired by miPSCs.

\section{Introduction}

Induced pluripotent stem cells (iPSCs) resemble embryonic stem cells (ESCs) in their near unlimited capacity for self-renewal and differentiation potential ${ }^{1}$. These properties have driven widespread uptake of iPSCs in clinical and research applications ${ }^{2-4}$. Despite their immense therapeutic promise, the reprogramming process required to generate iPSCs can produce genomic and epigenomic aberrations ${ }^{4-8}$. These abnormalities could undermine the functional equivalence of iPSCs and ESCs, or alter the phenotype of iPSC-derived differentiated cells, and hence necessitate genetic and functional screening of iPSCs prior to their use in the clinic ${ }^{9}$. Fortunately, whole genome sequencing (WGS) based analyses of single nucleotide variants (SNVs), copy number variants, and structural variants (SVs) restricted to human and mouse iPSC lines have found relatively few conclusive reprogramming-associated mutations ${ }^{10-12}$. Instead, most mutations acquired by iPSCs appear to occur before and after reprogramming ${ }^{10,11,13}$, implying they are not

27 caused by molecular processes inherent to iPSC generation. Transposable elements (TEs) may present an important exception to this rule, where the attainment of a pluripotent state via reprogramming leaves iPSCs vulnerable to TE-mediated mutagenesis.

The retrotransposon long interspersed element 1 (LINE-1, or L1) is active in nearly all 
transcription (TPRT), which involves reverse transcription of L1 mRNA in cis, and is characterized by the generation of target site duplications (TSDs) upon L1 integration ${ }^{15-20}$. The C57BL/6 mouse reference genome contains $\sim 3,000$ potentially mobile L1 copies belonging to three subfamilies $\left(\mathrm{T}_{\mathrm{F}}, \mathrm{G}_{\mathrm{F}}\right.$ and $\left.\mathrm{A}\right)$ defined by their monomeric $5^{\prime}$ promoter sequences, in addition to several active endogenous retrovirus (ERV) and short interspersed element (SINE) families ${ }^{21-}$ 23. By contrast, only $\sim 100$ mobile L1s from the transcribed subset Ta $(-\mathrm{Ta})^{24}$ subfamily are present in each individual human genome, with the vast majority of retrotransposition potential concentrated in fewer than 10 of these elements ${ }^{25,26}$. Perhaps owing to the disparate count of mobile TEs in each species, the rate of L1 mobilization in the mouse germline is estimated to be at least an order of magnitude higher than that of humans ${ }^{27-30}$.

TE mobility is regulated by DNA methylation and histone modifications, as well as various post-transcriptional and post-translational mechanisms ${ }^{31-41}$. Reprogramming somatic cells to generate human iPSCs (hiPSCs) and mouse iPSCs (miPSCs) leads to epigenome-wide remodeling, including broad de-repression of L1 promoters ${ }^{7,42-47}$. L1 mRNA transcription increases strongly during reprogramming, and remains approximately 10-fold higher in cultured miPSCs than in parental mouse embryonic fibroblasts $(\mathrm{MEFs})^{46}$. As a corollary, the early mouse embryo is a major niche for new heritable L1 retrotransposition events ${ }^{28}$. Mouse ESCs cultured in standard media containing serum express endogenous L1 proteins and support engineered L1 mobilization ${ }^{41}$. Naïve ESCs grown in media containing two small-molecule kinase inhibitors (2i) in place of serum also exhibit L1 promoter hypomethylation ${ }^{37,48}$. Engineered and endogenous L1 retrotransposition

52 are supported by hiPSCs and $\mathrm{ESCs}^{45,49-51}$. Collectively, these observations suggest L1

53 hypomethylation may be an intrinsic aspect of pluripotency accentuated by the molecular roadmap

54 to an induced pluripotent state. Consequently, miPSCs are likely to harbor de novo 55 retrotransposition events. A prior WGS analysis of 3 miPSC lines, employing paired-end 42mer reads and $\sim 11 \times$ genome-wide sequencing depth, however found no de novo TE insertions, and concluded that endogenous retrotransposition did not occur during miPSC production ${ }^{12}$. The

58 apparent lack of TE mobility in this context remains an unresolved and yet potentially important source of miPSC mutagenesis ${ }^{4}$. 
To survey genomic variation among miPSC lines generated from a broad range of parental cell types, we bred triple transgenic C57BL/6×129S4Sv/Jae animals carrying a GFP reporter knocked into the Oct4 locus (Oct4-GFP), a transcriptional activator (m2rtTA) under the control of the ubiquitously expressed Rosa26 locus (R26-m2rtTA), and a doxycycline-inducible polycistronic reprogramming cassette (Col1a1-tetO-OKSM) ${ }^{52}$. From each of three animals (labeled A67, A82 and A172), we used fluorescence activated cell sorting (FACS) and a range of surface markers to isolate nine isogenic primary cell populations, including three representing each germ layer (Fig. 1a). Bulk cultures were then treated with doxycycline to induce reprogramming, followed by FACS to purify Oct4-GFP ${ }^{+}$miPSCs. Twenty-six miPSC lines were successfully expanded and cultured in standard media containing serum (Supplementary Table 1 and Extended Data Fig. 1). Illumina paired-end 150 mer read WGS ( 41× average genome-wide depth) was then applied to each miPSC line at passage $4(\mathrm{p} 4)$, as well as to $3 \mathrm{MEF}$ genotypic controls (Supplementary Table 1).

Concordant SNVs detected by GATK HaplotypeCaller and freebayes ${ }^{53,54}$ were filtered to remove known mouse strain germline variants ${ }^{55}$, yielding 3,603 SNVs private to a single miPSC line (average 140 per line) (Supplementary Table 2). Of these, 27 in total were non-synonymous exonic mutations (Supplementary Table 2). We then called concordant SVs using Delly and GRIDSS ${ }^{56,57}$, finding 34 private SVs ( 1 per line). These included a $210 \mathrm{kbp}$ deletion of the de novo methyltransferase Dnmt3a in miPSCs derived from the hematopoietic stem cells of animal A172 (Supplementary Table 2). Considering private SNVs and SVs together, we observed no significant ( $\mathrm{p}<0.05$, one-way ANOVA with Tukey's multiple comparison test) difference in miPSC variant counts associated with parental cell type or germ layer, and SNV and SV rates resembled those found previously for fibroblast-derived miPSCs ${ }^{10,12}$. This result broadly suggested that choice of primary cell type, at least among the diverse panel assembled here, may not

\section{Bulk miPSC populations harbor de novo $\mathrm{L} 1$ insertions}

As de novo TE insertions can be overlooked by generalized SV calling algorithms ${ }^{58}$, we used TEBreak $^{59}$ to identify non-reference TE insertions. Known non-reference genome TE insertions ${ }^{55}$, 92 and those found in MEF genotypic controls or multiple miPSC lines, were filtered, leaving 4 
a

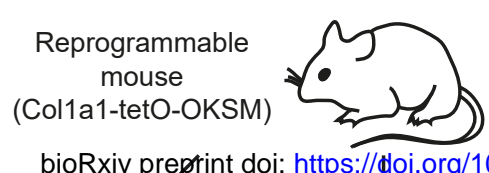

bioRxiv preprint doi: https://申oi.org/10.110/2022.02.16.480772; this version posted February 17, 2022. The copyright holder for this preprint

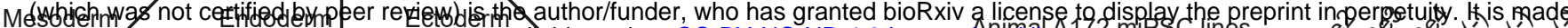

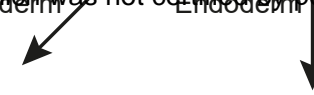

Granulocytes (1)

(2) Thymic epithelial cells (5) Fibroblasts (3) Intestinal stem cells (6)
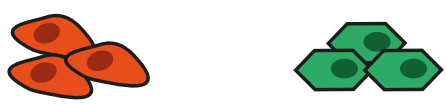

Reprogramming, miPSC colony formation, expansion
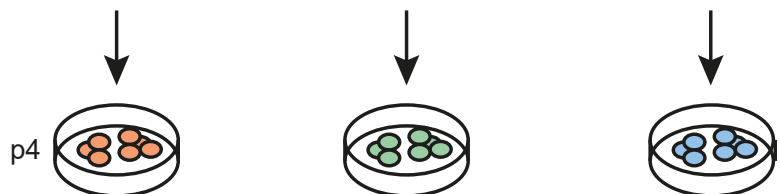

Astrocytes (7)

Keratinocytes (8)

Bulge stem cells $(9)$

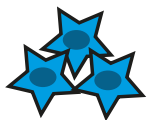

C

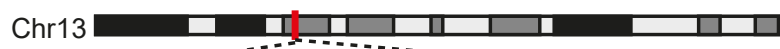

$\operatorname{miPSC} 3 \mathrm{~L}_{\mathrm{TF}}$

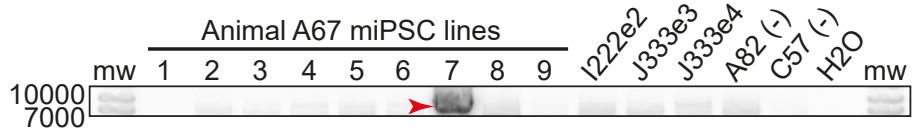

e

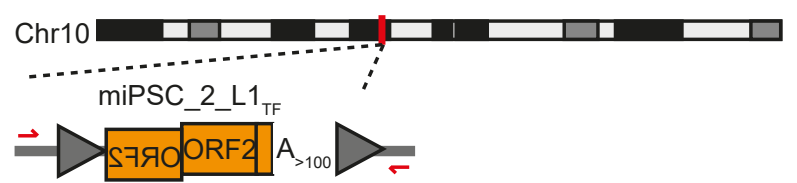

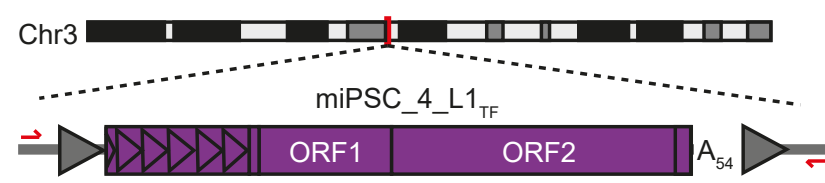

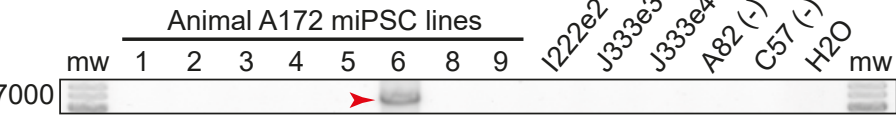

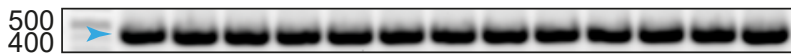

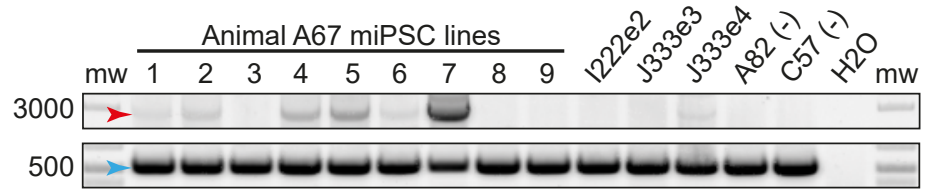

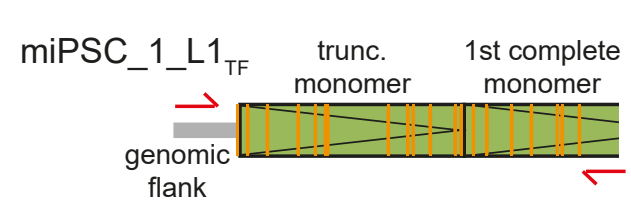

g

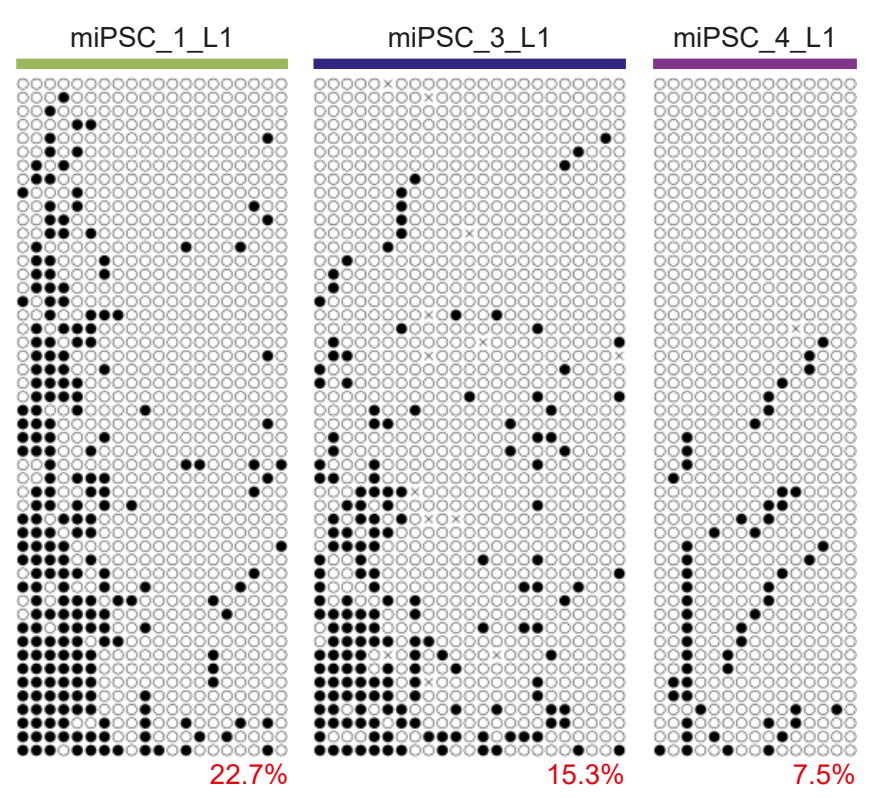

h
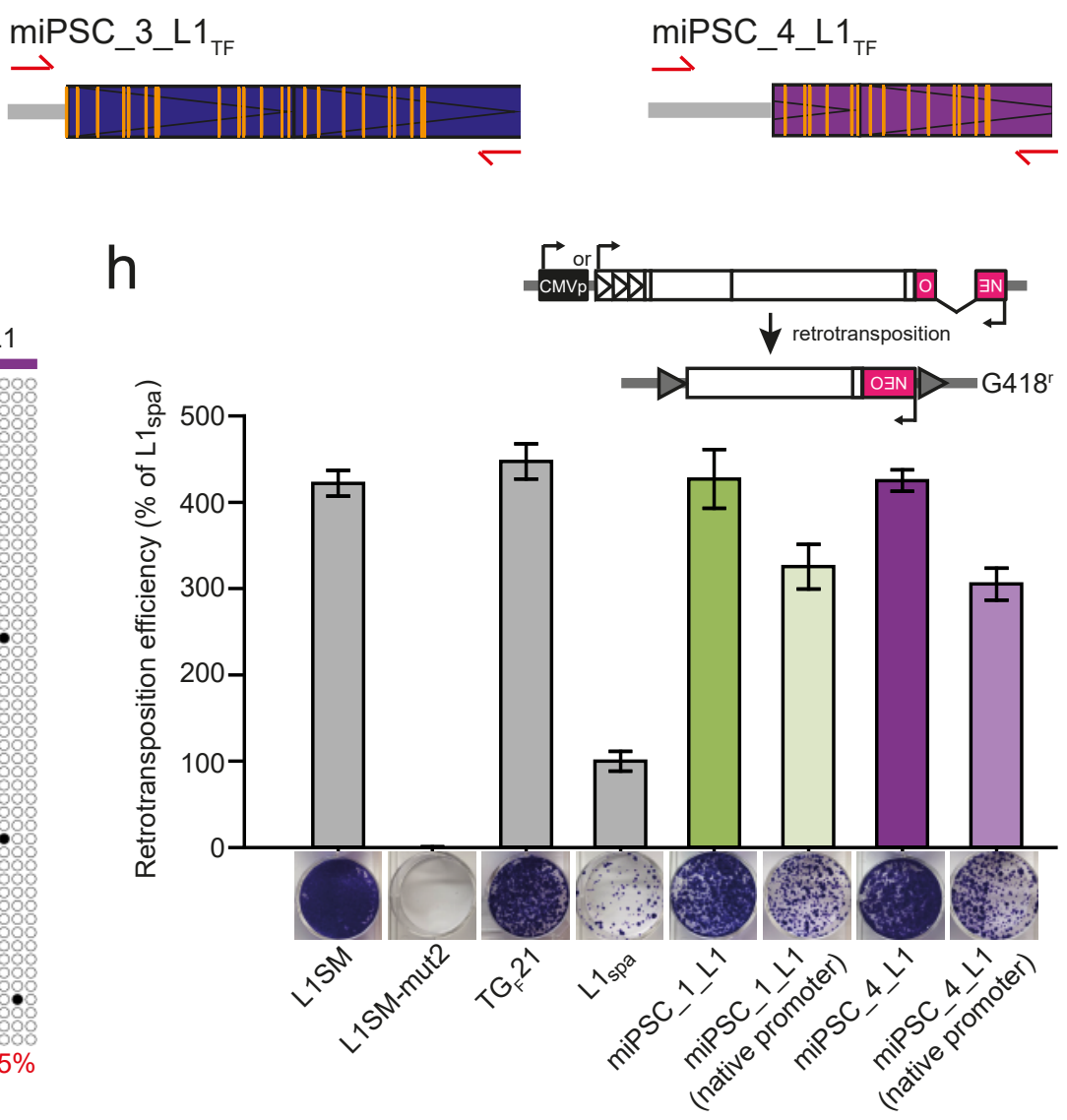
Table 1: De novo TE insertions detected in miPSC lines by Illumina sequencing.

\begin{tabular}{|c|c|c|c|c|c|c|c|}
\hline Insertion \# & Subfamily & Location & Monomers & Cleavage & TSD (bp) & PolyA (bp) & Origin \\
\hline miPSC_1_L1 & $\mathrm{T}_{\mathrm{F}}$ & $1 \mathrm{q}$ & 6.8 & TCTT/AG & 16 & $\sim 125$ & Reprogramming \\
\hline miPSC_2_L1 & $\mathrm{T}_{\mathrm{F}}$ & $10 \mathrm{q}$ & 0 & TTCT/GT & 14 & $>100$ & Mosaic \\
\hline miPSC_3_L1 & $\mathrm{T}_{\mathrm{F}}$ & $13 q$ & 5.8 & ATTC/AA & 15 & $\sim 50$ & Reprogramming \\
\hline miPSC_4_L1 & $\mathrm{T}_{\mathrm{F}}$ & $3 q$ & 5.3 & TCTT/AA & 13 & $\sim 54$ & Reprogramming \\
\hline miPSC_5_L1 & $\mathrm{GF}_{\mathrm{F}}$ & $19 q$ & 2 & TTAT/AT & 14 & $\sim 50$ & Reprogramming \\
\hline miPSC_6_L1 & $\mathrm{T}_{\mathrm{F}}$ & $7 q$ & 0 & TTTA/AA & 17 & $\sim 51$ & Reprogramming \\
\hline miPSC_7_L1 & $\mathrm{G}_{\mathrm{F}}$ & $\mathrm{Xq}$ & 5 & TCTT/AT & 16 & $>80$ & Reprogramming \\
\hline miPSC_8_L1 & $\mathrm{T}_{\mathrm{F}}$ & $19 q$ & 3.7 & TTTC/AA & 19 & $\sim 24$ & Reprogramming \\
\hline miPSC_9_B2 & B2 & $11 q$ & NA & TCTT/AC & 16 & $>60$ & Reprogramming \\
\hline miPSC_10_L1 & $\mathrm{T}_{\mathrm{F}}$ & $12 q$ & 0 & TTTT/GT & 6 & $\sim 36^{*}$ & Reprogramming \\
\hline miPSC_11_B2 & B2 & $13 q$ & NA & TTTT/GA & 14 & $>73$ & Reprogramming \\
\hline miPSC_12_L1 & $\mathrm{T}_{\mathrm{F}}$ & $13 q$ & 0 & TCTT/AG & 17 & $\sim 97$ & Reprogramming \\
\hline miPSC_13_L1 & A & $14 q$ & 3 & TTTC/AT & 13 & $\sim 46$ & Reprogramming \\
\hline miPSC_14_B2 & B2 & $15 q$ & NA & TTTT/AC & 16 & $>66$ & Reprogramming \\
\hline miPSC_15_L1 & $\mathrm{G}_{\mathrm{F}}$ & $2 q$ & 0 & TTTC/AA & 17 & $\sim 28^{*}$ & Reprogramming \\
\hline miPSC_16_L1 & $\mathrm{T}_{\mathrm{F}}$ & $2 q$ & $>3$ & TTTT/AA & 16 & $>100$ & Reprogramming \\
\hline miPSC_17_L1 & $\mathrm{T}_{\mathrm{F}}$ & $3 q$ & $>3$ & ACTT/AA & 14 & $\sim 45$ & Reprogramming \\
\hline miPSC_18_B1 & B1 & $3 q$ & NA & TTTT/AA & 15 & $\sim 30$ & Reprogramming \\
\hline miPSC_19_L1 & $\mathrm{T}_{\mathrm{F}}$ & $3 q$ & $>3$ & GTTT/AT & 15 & $>80$ & Reprogramming \\
\hline miPSC_21_L1 & $\mathrm{T}_{\mathrm{F}}$ & $4 q$ & 0 & TTTT/CA & 17 & $>150$ & Reprogramming \\
\hline miPSC_22_B2 & B2 & $6 q$ & NA & TCTT/GA & 15 & $\sim 52$ & Reprogramming \\
\hline miPSC_23_B2 & B2 & $9 q$ & NA & TTTT/AT & 16 & $\sim 50$ & Mosaic \\
\hline miPSC_24_B2 & B2 & $\mathrm{Xq}$ & NA & TTTT/AA & 15 & $>100$ & Reprogramming \\
\hline miPSC_26_L1 & $\mathrm{T}_{\mathrm{F}}$ & $1 q$ & $>3$ & TCTT/AT & 22 & $\sim 58$ & Reprogramming \\
\hline miPSC_27_B2 & B2 & $11 \mathrm{q}$ & NA & TTTC/AA & 14 & $>60$ & Reprogramming \\
\hline miPSC_28_L1 & $\mathrm{T}_{\mathrm{F}}$ & $13 q$ & 3.6 & TCCT/AA & 15 & $\sim 93 *$ & Reprogramming \\
\hline miPSC_29_L1 & $\mathrm{T}_{\mathrm{F}}$ & $15 \mathrm{q}$ & 0 & TCTT/AA & 16 & $>80$ & Reprogramming \\
\hline miPSC_30_L1 & $\mathrm{T}_{\mathrm{F}}$ & $6 q$ & $>3$ & TCTT/AT & 16 & $\sim 72$ & Reprogramming \\
\hline miPSC_31_L1 & $\mathrm{T}_{\mathrm{F}}$ & $7 q$ & $>3$ & TTTG/AC & 15 & $\sim 43$ & Reprogramming \\
\hline miPSC_32_L1 & $\mathrm{T}_{\mathrm{F}}$ & $\mathrm{Xq}$ & 2 & TCTT/AT & 13 & $\sim 37$ & Reprogramming \\
\hline miPSC_33_L1 & $\mathrm{GF}_{\mathrm{F}}$ & $\mathrm{Xq}$ & $>3$ & TTTT/AA & 15 & $\sim 47$ & Reprogramming \\
\hline miPSC_34_L1 & $\mathrm{T}_{\mathrm{F}}$ & $8 \mathrm{q}$ & 0 & TCTT/AA & 6 & $\sim 36^{*}$ & Reprogramming \\
\hline miPSC_35_L1 & $\mathrm{T}_{\mathrm{F}}$ & $1 \mathrm{q}$ & 0 & TTTA/AA & 15 & $\sim 38$ & Reprogramming \\
\hline miPSC_36_L1 & $\mathrm{G}_{\mathrm{F}}$ & $8 \mathrm{q}$ & 0 & ATGT/GA & 6 & $\sim 42$ & Reprogramming \\
\hline miPSC_37_L1 & $\mathrm{T}_{\mathrm{F}}$ & $1 \mathrm{q}$ & 1.2 & TTTT/GT & 14 & $\sim 20$ & Reprogramming \\
\hline miPSC_38_L1 & $\mathrm{T}_{\mathrm{F}}$ & $10 q$ & 0 & TTCT/AA & 15 & $\sim 55$ & Reprogramming \\
\hline miPSC_39_L1 & $\mathrm{T}_{\mathrm{F}}$ & $10 q$ & 0 & TTTT/AA & 8 & $>140^{*}$ & Reprogramming \\
\hline miPSC_40_L1 & $\mathrm{T}_{\mathrm{F}}$ & $11 \mathrm{q}$ & $>3$ & TTTT/GA & 14 & $>120$ & Reprogramming \\
\hline miPSC_41_L1 & $\mathrm{T}_{\mathrm{F}}$ & $12 \mathrm{q}$ & 2.6 & TCTT/GC & 16 & $\sim 49$ & Reprogramming \\
\hline miPSC_42_B1 & B1 & $14 \mathrm{q}$ & NA & TTCT/AA & 15 & $>50$ & Reprogramming \\
\hline miPSC_43_L1 & $\mathrm{T}_{\mathrm{F}}$ & $16 \mathrm{q}$ & $>3$ & ATTT/AA & 14 & $\sim 42 *$ & Mosaic \\
\hline
\end{tabular}

Monomers: number of monomeric promoter units found for full-length L1 insertions. Cleavage: L1 endonuclease cleavage motif. TSD: target site duplication length. PolyA: polyA tract length estimated by Sanger sequencing. Insertions marked with an asterisk carry a 3' transduction. Note: miPSC_1_L1 - miPSC_8_L1 were detected in bulk miPSCs; the remaining insertions were detected in single-cell miPSC clones. 
Table 3). To achieve even greater coverage of potentially active TEs, we performed mouse retrotransposon capture sequencing (mRC-seq), which uses sequence capture probes to enrich Illumina libraries for the $5^{\prime}$ and $3^{\prime}$ genomic junctions of mobile TEs, including $\mathrm{T}_{\mathrm{F}}, \mathrm{G}_{\mathrm{F}}$ and $\mathrm{A}$ subfamily L1s, B1 and B2 SINEs, and IAP and ETn ERVs (Supplementary Table 1) ${ }^{28,60}$. The combination of WGS and mRC-seq identified an additional 4 putative de novo $\mathrm{L}_{1} \mathrm{G}_{\mathrm{F}}$ and $\mathrm{T}_{\mathrm{F}}$ insertions (Extended Data Fig. 3, Table 1 and Supplementary Table 3).

We PCR amplified and fully characterized each putative L1 insertion sequence. Six events were full-length, retaining 2-7 monomers at their 5' end, and could only be amplified in the miPSC line where they were detected by genomic analysis (Fig. 1b-d, Extended Data Fig. 2 and Extended Data Fig. 3). An additional L1 (labeled miPSC_6_L1) was very heavily 5' truncated and confirmed by PCR to be private to one miPSC line (Extended Data Fig. 3). The final example (miPSC_2_L1) was heavily 5' truncated and inverted ${ }^{61}$ and could be PCR amplified in 7/9 miPSC lines representing all 3 germ layers of animal A67 (Fig. 1e and Extended Data Fig. 2). miPSC_2_L1 most likely represented a mosaic insertion that arose early in the embryonic development of animal A67, as found previously ${ }^{28,30,62,63}$. Each insertion carried TSDs of 13-19nt, a long and pure $3^{\prime}$ polyA tract, and integrated at a degenerate L1 endonuclease recognition motif (5'-TTTT/AA-3') (Table 1). These hallmarks were consistent with bona fide TPRT-mediated L1 retrotransposition events ${ }^{16,19,64,65}$. In sum, 10/26 miPSC lines harbored at least one PCR validated de novo L1 insertion. Not counting the mosaic miPSC_2_L1 insertion, miPSCs from all 3 animals and 4/9 cell types, representing each germ layer, presented at least one de novo L1 insertion (Supplementary Table 3). Notably, down-sampling to $11 \times$ depth WGS, as $\operatorname{per}^{12}$, indicated an expected $95 \%$ probability of finding none of the validated de novo insertions (Extended Data Fig. 4a).

Comprehensive capillary sequencing of the 3 full-length insertions (miPSC_1_L1, miPSC_3_L1 and miPSC_4_L1) revealed that each had intact ORFs (Fig. 1b-d). To assess the potential for further mobilization of these newly retrotransposed elements, we first used multiplexed L1 locus-specific bisulfite sequencing ${ }^{34,60}$ to measure $\mathrm{CpG}$ methylation of their most 5' promoter monomers (Fig. 1e). All 3 full-length elements were fully unmethylated in a subset of miPSCs, and their methylation decreased with distance from the L1 5 ' end (Fig. 1g). Next, we cloned and tested miPSC_1_L1 and miPSC_4_L1 in a cultured cell retrotransposition assay ${ }^{19,66}$, using the natural elements $\mathrm{L}_{\mathrm{spa}}\left(\mathrm{T}_{\mathrm{F}} \text { subfamily }\right)^{22}$ and $\mathrm{TGF}_{\mathrm{F}} 21$ (GF $_{\mathrm{F}}$ subfamily ${ }^{21}$ as positive controls, 
125

126

127

128

129

130

131

132

133

134

135

136

137

138

139

140

141

142

143

144

145

146

147

148

149

150

151

152

153

154

155

as well as the highly mobile synthetic L1 TF element L1SM ${ }^{67}$. miPSC_1_L1 and miPSC_4_L1 retrotransposed efficiently (Fig. 1h) when expressed from their native promoter or a cytomegalovirus promoter. Thus, endogenous L1 mobilization in miPSCs is driven by highly active donor L1s that can produce offspring L1s that are incompletely methylated and retrotransposition-competent.

\section{Single-cell miPSC clones reveal extensive L1-mediated endogenous retrotransposition}

Despite de novo L1 insertions being present in 10/26 miPSC lines, we were concerned that the heterogeneous mixture of cellular clones contained in bulk reprogrammed miPSCs could obscure TE insertions. We therefore reprogrammed MEFs from one of our C57BL/6×129S4Sv/Jae animals (labeled I222e2), isolated individual miPSCs via FACS, and expanded 18 clones cultivated in serum until p3, then in serum or 2i (naïve) culture conditions until p6 (Extended Data Fig. 2a). We then applied $\sim 41 \times$ average genome-wide depth Illumina WGS and mRC-seq to miPSC singlecell clones 1-9, and mRC-seq only to clones 10-18, with each clone analyzed after culture in serum or 2i media (Fig. 2a, Extended Data Fig. 1 and Supplementary Table 1). Deep WGS was performed on the parental I222e 2 MEF population, attaining cumulative $117 \times$ genome-wide depth, in addition to mRC-seq (Supplementary Table 1). Using the WGS data, we again called concordant SNVs and SVs private to one miPSC clone, while excluding known germline variants and those found in the parental MEFs. We found, on average, $\sim 100$ and $\sim 1$ private SNVs and SVs per miPSC clone, respectively, almost all of which were detected in both the serum and $2 \mathrm{i}$ conditions for each clone (Supplementary Table 2). These frequencies resembled those found by genomic analysis of bulk miPSCs, underlining that heterogeneous and homogeneous fibroblastderived miPSC populations are relatively free of genomic abnormalities ${ }^{10,12}$. This experiment also indicated choice of serum or $2 \mathrm{i}$ media did not impact the frequency of SNVs or SVs present in miPSCs.

By contrast, TEBreak revealed 35 putative de novo TE insertions absent from the parental MEFs, all of which were found in both serum and $2 \mathrm{i}$ culture conditions for at least one miPSC clone. Of these, 27 were detected by both WGS and mRC-seq, 6 by mRC-seq only, and 2 by WGS only (Supplementary Table 3). We were able to PCR amplify 32 insertions in full and capillary sequence at least their 5' and 3' junctions (Fig. 2b-f, Extended Data Fig. 3 and Supplementary

Table 3). Two other putative TE insertions could only be amplified at their $5^{\prime}$ genome junction; 


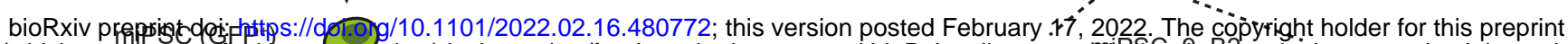

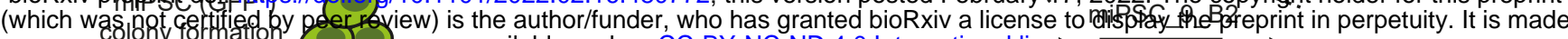

Single cell isolation (FACS)

p0

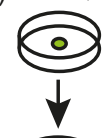

Cell expansion and cultivation $\mathrm{p} 3$
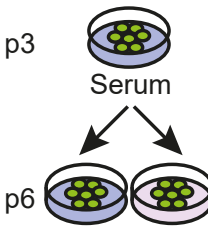

Serum $2 \mathrm{i}$

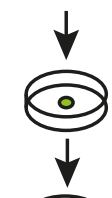

$\searrow$

4
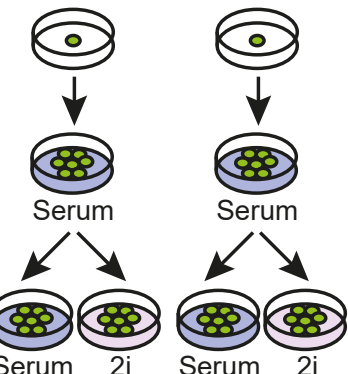

Serum $2 i$ serum $2 i$

C
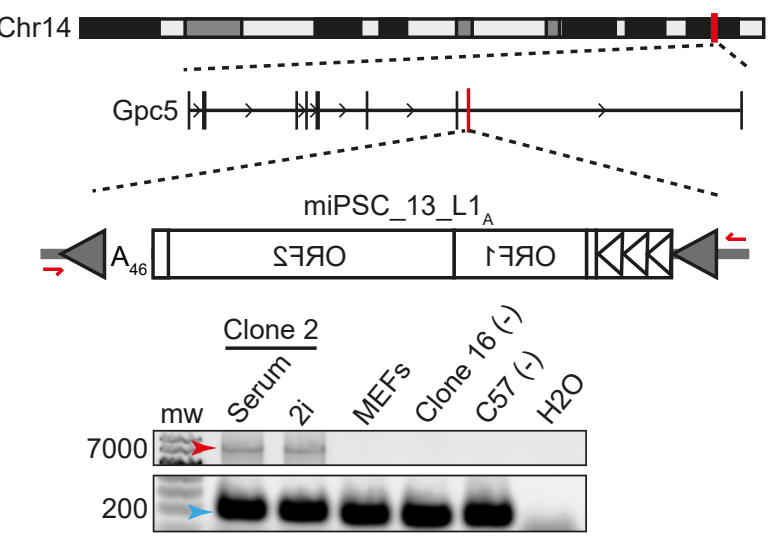

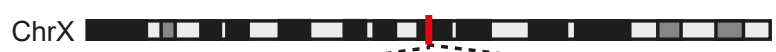
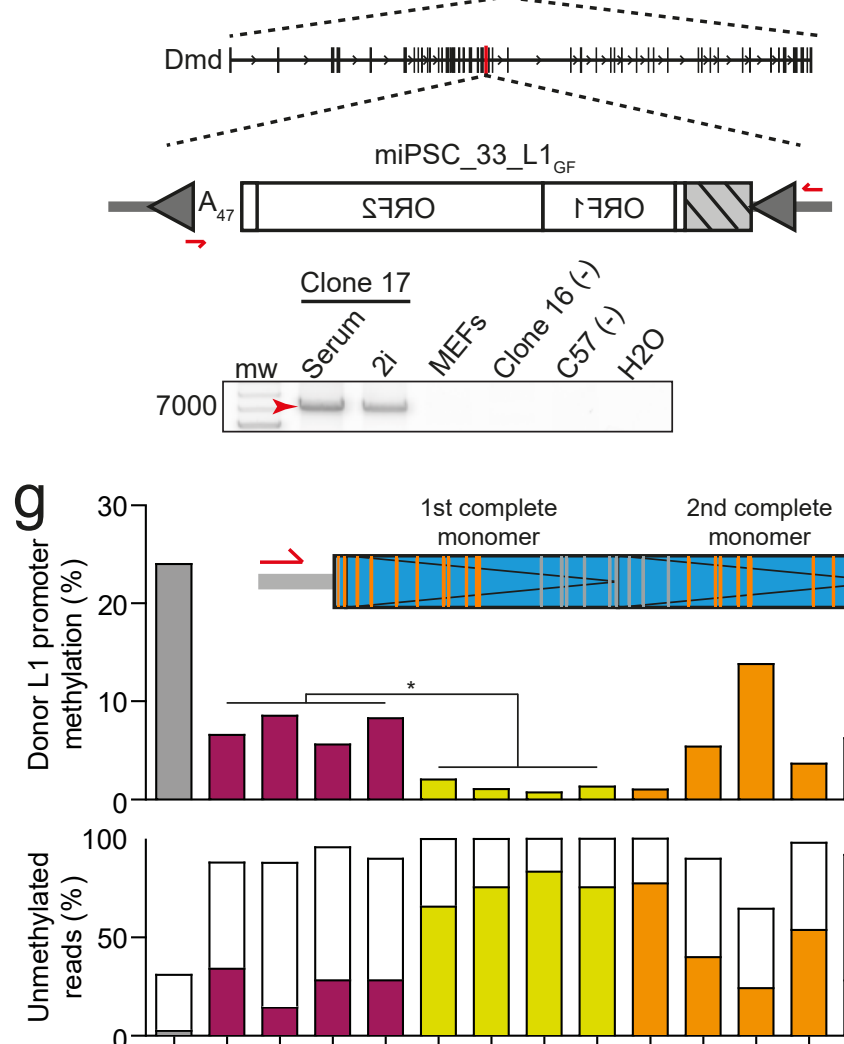

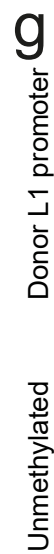
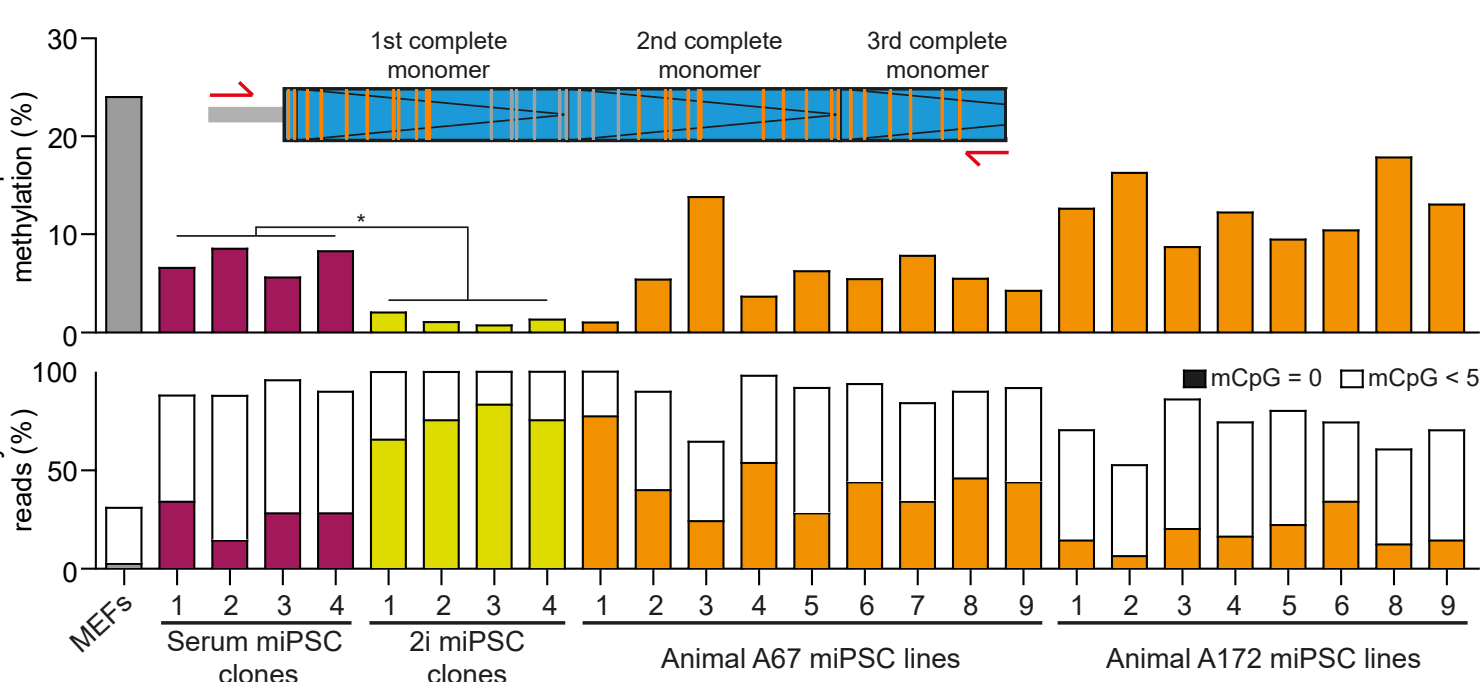
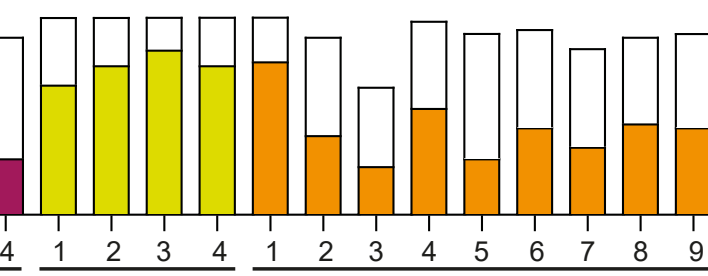
clones

\begin{abstract}
\begin{tabular}{lllllllll}
4 & 1 & 2 & 3 & 4 & 5 & 6 & 7 & 8 \\
\cline { 3 - 4 } & Animal A67 miPSC lines
\end{tabular}
\end{abstract}

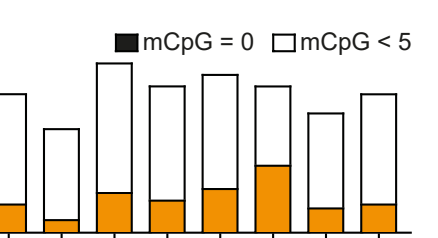
clones
clom clones

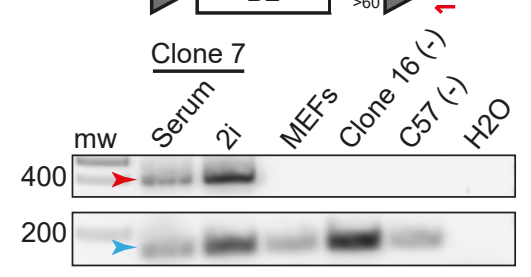

d crosing

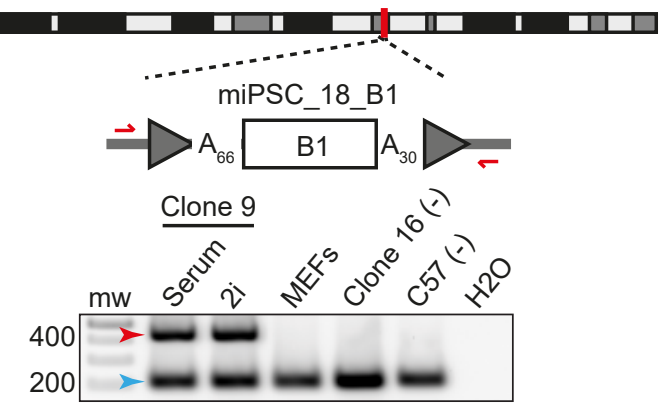

f Chr12

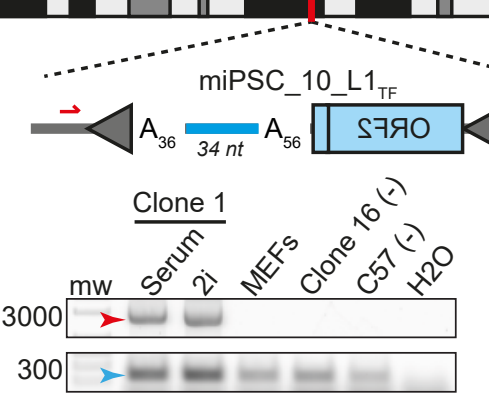

Retrotransposition
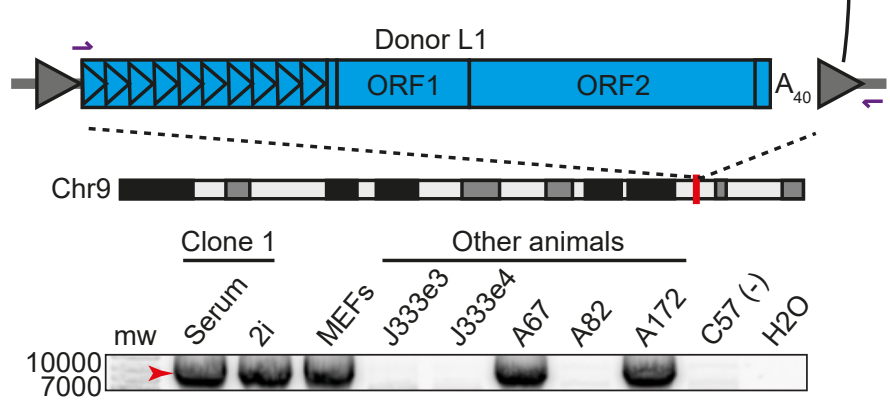

h

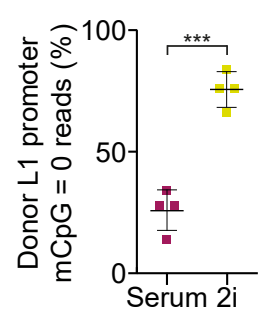

i

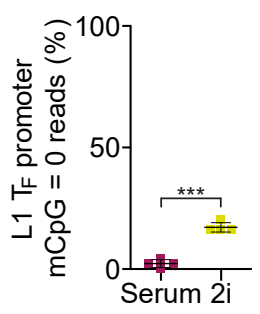


Fig. 2: Frequent de novo TE insertions in MEF-derived clonally expanded miPSC lines. a, Experimental design to generate single-cell miPSC clonal lines. Bulk MEFs from a Col1a1-tetO-OKSM mouse (animal I222e2) were purified and reprogrammed by addition of doxycycline. Individual Oct4-GFP positive miPSCs were then isolated via FACS, expanded in serum for 3 passages,

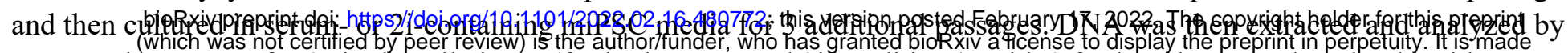

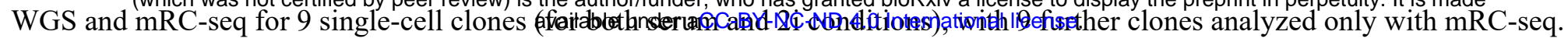
b, A full-length de novo B2 inserted and orientated in antisense to intron 15 of Brca1. PolyA tract length is indicated immediately 3' of the B2. TSDs are depicted as grey arrows flanking the B2. PCR validation (gel pictures shown) involved an empty/filled PCR design where both primers (red arrows) are outside of the B2, generating "filled" B2 (red arrow) and "empty" wild-type (blue arrow) products. The B2 was amplified only in either the serum or $2 \mathrm{i}$ conditions for the single-cell clone (number 7) where the B2 was detected by genomic analysis, and not in the matched parental MEFs, the C57BL/6 strain, or a single-cell clone (number 16) selected at random. c, A full-length (3 monomers) L1 A subfamily element inserted de novo antisense to intron 7 of Gpc5. Sequence characteristics and PCR validation results are shown as in panel (b). Promoter monomers are shown as triangles within the L1 5'UTR. d, As in panel (b), except showing an unusual intergenic B1 insertion flanked by both 5' and 3' polyA tracts. e, A full-length $\mathrm{L} 1 \mathrm{G}_{\mathrm{F}}$ inserted de novo antisense to intron 60 of $\mathrm{Dmd}$. $\mathrm{PCR}$ validation involved a $5^{\prime}$ genomic primer and a 3' junction primer (red arrows). As indicated by a grey box with black stripes, the number of monomers is unknown but was $>3$. f, A heavily $5^{\prime}$ truncated, intergenic de novo $\mathrm{L} 1 \mathrm{~T}_{\mathrm{F}}$ insertion validated by empty/filled PCR, as per panel (b). Sequence features are annotated as per panel (b), with the addition of a 34nt 3' transduction matching a donor $\mathrm{L}_{1} \mathrm{~T}_{\mathrm{F}}$ located on Chromosome 9. PCR using primers (purple arrows) designed to amplify the entire donor L1 indicated it was polymorphic in our colony. Capillary sequencing indicated the donor L1 retained a promoter of 10 monomers and had intact ORFs. g, Locus-specific bisulfite sequencing analysis of the donor L1 promoter identified in panel (f), in MEFs, single-cell miPSC clones, and miPSC lines derived from primary cells. top: Assay design and primer locations. CpGs located in the first 3 monomers of the donor L1 were assessed. Orange and grey strokes indicate CpGs covered and not covered, respectively, by sequencing the amplicon with $2 \times 300$ mer Illumina reads. middle: Mean percentages of donor L1 CpG methylation for 50 non-identical sequences selected at random from each sample. A two-tailed t test $(* \mathrm{p}<0.05)$ was used to compare serum and $2 \mathrm{i}$ culture conditions for single-cell miPSC clones 1-4. bottom: Percentages of fully unmethylated ( $\mathrm{mCpG}=0$, filled bars) and heavily unmethylated $(0<\mathrm{mCpG}<5$, white bars) reads using the same sequencing data as displayed in the above histogram. $\mathbf{h}$, Percentages of fully unmethylated $(\mathrm{mCpG}=0)$ reads corresponding to the donor L1 promoter identified in panel (f), for miPSCs cultured in serum or $2 \mathrm{i}$ conditions. Data represent mean methylation $\pm \mathrm{SD}$ observed for single-cell miPSC clones 1-4. Significance testing was via two-tailed $t$ test $(* * * p<0.0001)$. i, As for panel (h), except using an assay targeting the L1 $\mathrm{T}_{\mathrm{F}}$ subfamily monomer. 
one of these (miPSC_29_L1) however also had strong 3' WGS and mRC-seq support. We therefore considered 33 TE insertions as validated de novo events (Table 1 and Supplementary Table 3). Thirty-one of these were PCR validated as private to only one miPSC clone, whereas the remaining two events were found in either 2 clones (miPSC_23_B2) or 4 clones (miPSC_43_L1) (Extended Data Fig. 3). These last two insertions were therefore present in subclones of the parental MEF population.

The 33 fully characterized de novo insertions included 20, 3 and $1 \mathrm{~T}_{\mathrm{F}}, \mathrm{G}_{\mathrm{F}}$ and A L1 subfamily members, respectively, as well as 2 B1 and 7 B2 elements (Fig. 2b-f). All insertions generated TSDs and a 3' polyA tract, and integrated at a degenerate L1 endonuclease motif (Table 1). 14/24 L1 insertions retained at least one promoter monomer and were therefore considered full-length (Table 1). Of the remaining $10 \mathrm{L1}$ s, 3 were 5' inverted (Supplementary Table 3). One unusual B1 insertion, miPSC_18_B1, was flanked by 5' and 3' polyA tracts as well as TSDs (Fig. 2d), likely arising via a variant of TPRT ${ }^{68}$. While no TE insertions were found in protein-coding exons, 14 were intronic, including a B2 antisense to the tumor suppressor gene Brcal (Fig. 2b) and an L1 GF antisense to the dystrophin gene Dmd (Fig. 2e). 15/18 miPSC clones (83.3\%) harbored at least one fully characterized TE insertion, including all clones analyzed with both WGS and mRC-seq (Supplementary Table 1). Clone 2 contained the most (6) insertions. No de novo ERV insertions were found.

Among 277 high confidence heterozygous non-reference TE insertions (Supplementary Table 4) found in the parental MEF population, 97.0\% were detected on average in each miPSC clone surveyed with WGS and mRC-seq. Down-sampling followed by seeking at least one WGS read in support of these non-reference insertions suggested our approach would distinguish approximately 50\%, 95\% and 99\% of de novo TE insertions from pre-existing subclonal TE insertions present in 1\%, 5\% and 10\% of cells, respectively (Extended Data Fig. 4b). Consistently, only 2/33 PCR validated TE insertions in the miPSC clones were subclonal in the parental MEFs (Table 1, Extended Data Fig. 3). An additional down-sampling analysis indicated de novo TE insertions were likely to be detected at a lower average WGS depth in the single-cell miPSC clones than insertions found in the bulk miPSC experiments (Extended Data Fig. 4a), in agreement with the greater homogeneity of the clonal miPSC cultivars. Deep sequencing of miPSCs and parental MEFs therefore enabled reliable detection and distinction of TE insertions arising before and during reprogramming. 


\section{A polymorphic retrotransposition-competent $\mathrm{L} 1$ eludes methylation}

189

190

191

192

193

\section{Six L1 insertions carried 3' transductions (Table 1, Supplementary Table 3 and Extended Data}

Fig. 3), flanking sequences generated when PolII bypasses the native L1 polyA signal in favor of a downstream alternative ${ }^{69-73}$. Of these transductions, 5 were either too short to reliably map to the genome, or mapped to multiple locations (Supplementary Table 3). The remaining 34bp transduction accompanied a 5' truncated L1 TF insertion on Chromosome 12 (miPSC_10_L1) (Fig. 2f). While the transduction aligned uniquely to Chromosome 9, a donor L1 was not present adjacent to this reference genome location. However, PCR amplification revealed an L1 TF immediately upstream of the transduced sequence (Fig. 2f). This donor L1 was polymorphic in our $\mathrm{C} 57 \mathrm{BL} / 6 \times 129 \mathrm{~S} 4 \mathrm{~Sv} / \mathrm{Jae}$ animals and retained a $5^{\prime}$ promoter comprising an unusully high number of monomers (10). Capillary sequencing confirmed the donor L1 possessed intact ORFs. L1 locus-specific bisulfite sequencing revealed that few $(24.1 \%)$ of the $\mathrm{CpG}$ dinucleotides in the first two monomers of the donor L1 promoter were methylated in MEFs (Fig. 2g and Extended Data Fig. 5), as opposed to 7.3\% in a subset of single-cell miPSC clones cultured in serum, and $1.3 \%$ for the same miPSC clones when cultured in $2 \mathrm{i}$ conditions (Fig. 2g). This difference in CpG methylation between culture conditions was significant ( $\mathrm{p}<0.05$, two-tailed $t$ test). The donor L1 promoter was fully unmethylated in nearly all miPSCs cultured in 2i (Fig. 2g and Extended Data Fig. 5). Indeed, significantly more ( $<<0.0001$, two-tailed t test) fully unmethylated sequences were found for the donor L1 promoter in $2 \mathrm{i}$ conditions than in serum, possibly as a consequence of global naïve state hypomethylation (Fig. 2h). Among the bulk reprogrammed miPSCs obtained from animals A67 and A172, which carried the donor L1 (Fig. 2f), only 9.1\% of CpG dinucleotides were methylated in the donor L1 promoter, and fully unmethylated sequences were identified in all miPSC lines (Fig. 2g and Extended Data Fig. 5). By contrast, in MEFs, 83.6\% of CpG dinucleotides in L1 $\mathrm{T}_{\mathrm{F}}$ promoter monomers genome-wide were methylated, compared to $45.2 \%$ among the A67 and A172 miPSC lines (Extended Data Fig. 6). L1 TF subfamily monomers were also significantly ( $\mathrm{p}<0.001$, two-tailed t test) less methylated in $2 \mathrm{i}(34.3 \%)$ miPSC conditions than serum (53.5\%), leading to an increase in fully unmethylated monomers (Fig. 2i and Extended Data Fig. 6). These bisulfite sequencing analyses highlighted genome-wide and persistent relaxation of L1 $\mathrm{T}_{\mathrm{F}}$ methylation in miPSCs, leaving mobile L1 promoters completely unmethylated. 


\section{Reprogramming is unaffected by $L 1$ reverse transcriptase inhibition}

Lamivudine (3TC) is a potent nucleoside reverse transcriptase inhibitor known to limit engineered retrotransposition assays conducted in cultured HeLa cells, 3TC was tested at a maximum concentration of $25 \mu \mathrm{M}$ against the codon-optimized L1SM element, reducing its mobility by $\sim 50 \%{ }^{74}$. By performing titration experiments to optimize the use of 3TC during miPSC generation, we determined that $3 \mathrm{TC}$ concentrations of up to $100 \mu \mathrm{M}$ did not reduce MEF reprogramming efficiency (Fig. 3a and Extended Data Fig. 7a), or viability of cultured MEFs or miPSCs (Extended Data Fig. 7b). Using a wild-type L1 $\mathrm{T}_{\mathrm{F}}$ carrying an mCherry retrotransposition indicator cassette, we found $100 \mu \mathrm{M} 3 \mathrm{TC}$ reduced mouse L1 retrotransposition by $\sim 95 \%$ in $\mathrm{HeLa}$ cells (Fig. 3b). These data indicated 3TC may be used, without apparent drawbacks, to limit L1mediated mutagenesis arising during reprogramming and miPSC cultivation.

\section{Nanopore genomic analysis of TE insertions in bulk miPSCs}

In principle, a single long read can completely resolve a de novo $\mathrm{TE}$ insertion present in a heterogeneous cell population, as well as the accompanying TPRT hallmarks ${ }^{76}$. Long-read sequencing can also discover TE insertions in repetitive genomic regions refractory to mapping with short-read approaches ${ }^{35,77,78}$. We therefore applied Oxford Nanopore Technologies (ONT) PCR-free long-read sequencing ( $20 \times$ average genome-wide depth) to 4 bulk miPSC lines, 2 of which were reprogrammed in the presence of $100 \mu \mathrm{M} 3 \mathrm{TC}$, as well as matched parental MEFs (Fig. ONT data, we identified 3,879 non-reference TE insertions carried by the parental MEFs (Supplementary Table 4). Of these, 3,380 (87.1\%) corresponded to known insertions ${ }^{55}$. To gauge the general tractability of PCR validation applied to this dataset, we used a panel of 4 heterozygous non-reference TE insertions (Supplementary Table 3). All of these successfully amplified in the MEFs and miPSCs (Extended Data Fig. 7c).

An additional $16 \mathrm{TE}$ insertions were each detected in only one miPSC line and not the 247 least one ONT read fully spanning the integrated TE sequence (Supplementary Table 3). 


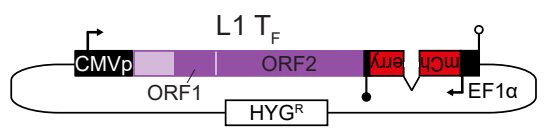

Bulk reprogramming

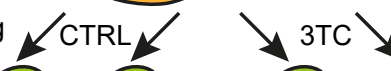

$\downarrow$ Retrotransposition

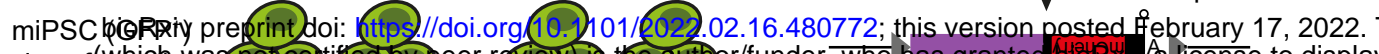

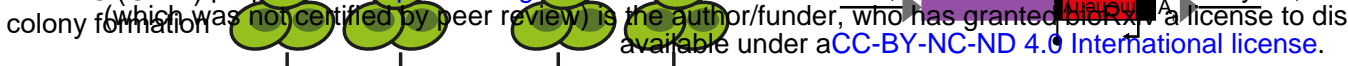

Cell expansion and cultivation (p1, serum)

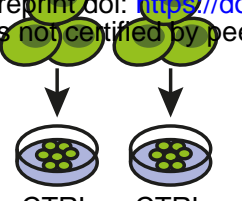

CTRL CTRL

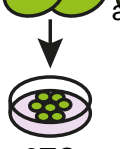

3TC
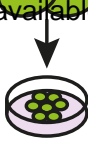

+ Hygromycin

$\mathrm{S} \mathrm{T} / \mathrm{O}-\mathrm{O}-\mathrm{O}-\mathrm{O}-\mathrm{O}-\mathrm{O}-\mathrm{O}-\mathrm{O}$

O-O $123 \quad 3 \quad 4 \quad 5 \quad 6 \quad 7$ R

$\mathrm{O}-\mathrm{O}-\mathrm{O}-\mathrm{O}-\mathrm{O}-\mathrm{O}-\mathrm{O}-\mathrm{O}$ + Hygromycin, $+3 \mathrm{TC}$

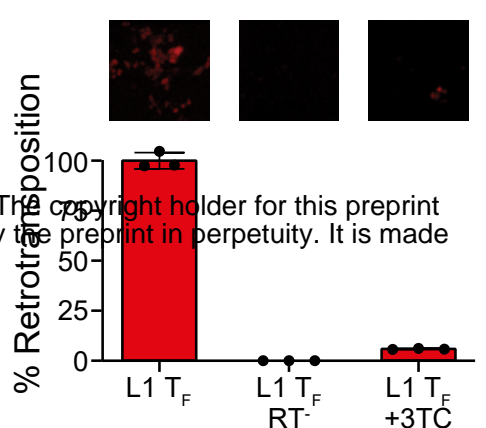

C
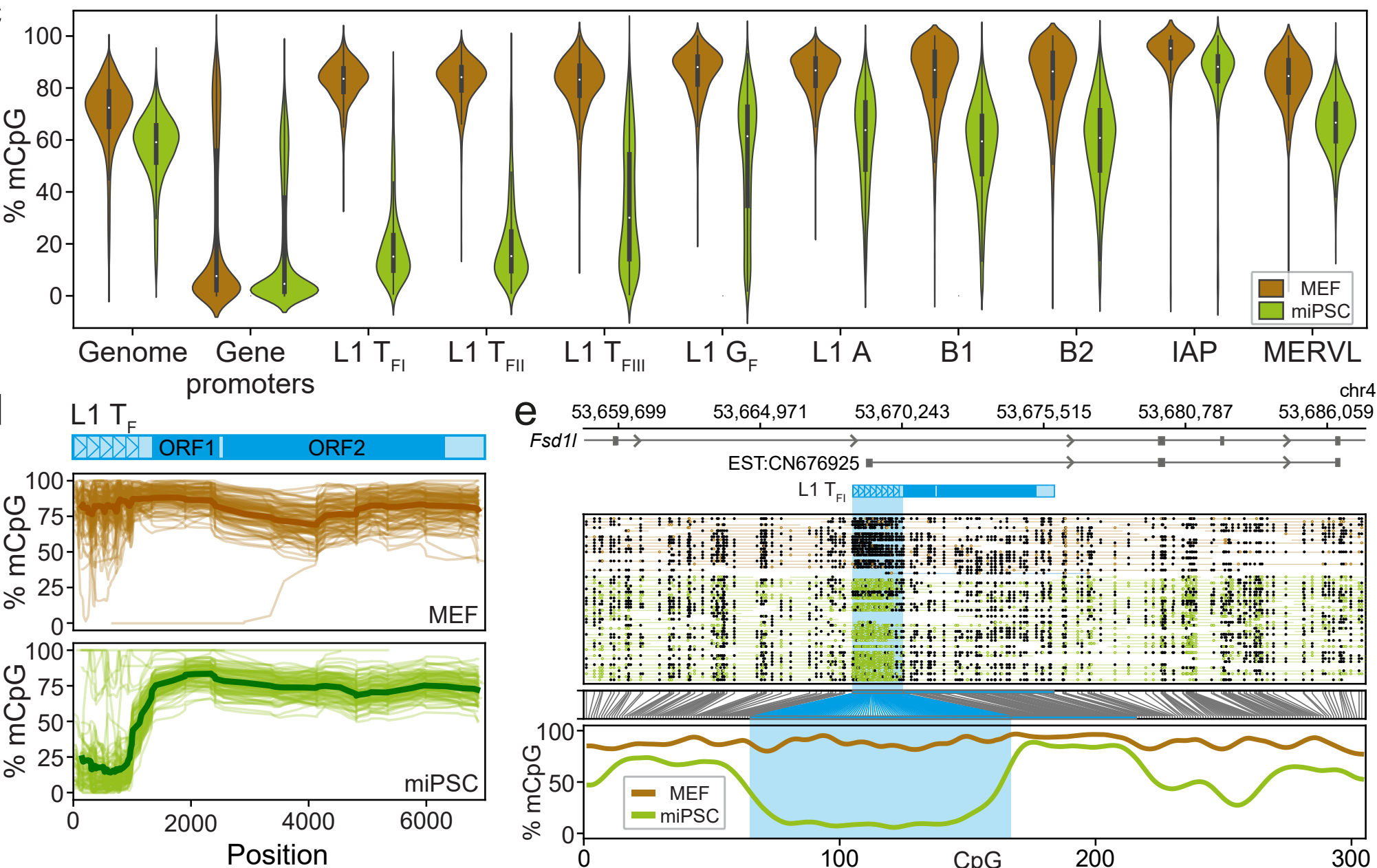

Fsd1I $53,659,699$

EST:CN676925 -

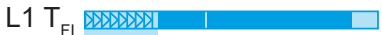

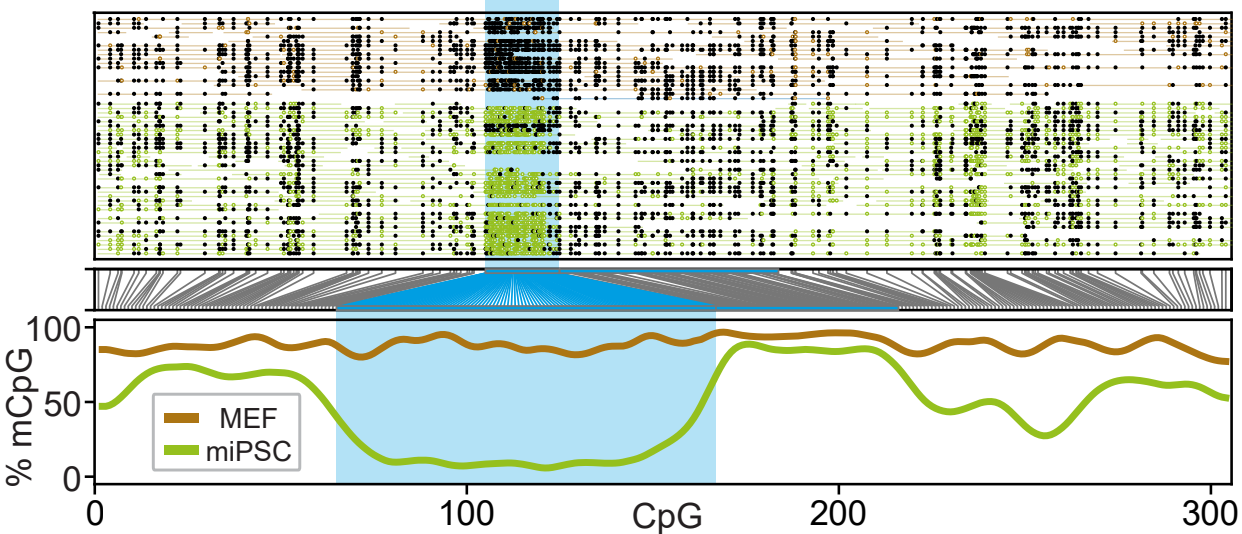

Fig. 3: Long-read genomic analysis of TE methylation and mobilization in MEF-derived bulk miPSC lines. a, Bulk MEFs

were reprogrammed by the addition of doxycycline. Oct4-GFP positive miPSCs were then sorted and expanded in serum. Two miPSC lines were reprogrammed and cultured in media containing $100 \mu \mathrm{M}$ lamivudine (3TC), and two lines generated without lamivudine (CTRL). DNA was extracted from MEFs and miPSCs and ONT sequenced. b, top left: retrotransposition indicator plasmid L1_mCherry consists of the pCEP4 backbone (CMV promoter, black; SV40 polyadenylation signal, open lollipop; hygromycin resistance gene, white) containing a wild-type L1 $\mathrm{T}_{\mathrm{F}}$ element (5'UTR, light purple; ORFs, dark purple). An mCherry reporter gene equipped with an EF1 $\alpha$ promoter and HSVtk polyadenylation signal (black lollipop) is inserted into the L1 3'UTR antisense to the $\mathrm{L} 1$. The mCherry sequence is interrupted by an intron in sense orientation relative to the L1, ensuring mCherry expression only upon retrotransposition. bottom left: retrotransposition assay timeline. Cells were split (S), transfected (T), and cultured in hygromycin-containing medium with and without $100 \mu \mathrm{M} 3 \mathrm{TC}$. Retrotransposition efficiency was assessed by flow cytometry 8 days post-transfection (R). top right: fluorescence microscopy images showing representative wells at 8 days post-transfection with L1 $\mathrm{T}_{\mathrm{F}}$ (left), reverse transcriptase mutant $\left(\mathrm{RT}^{-}\right) \mathrm{L}_{1} \mathrm{~T}_{\mathrm{F}}$ (middle), and $\mathrm{L}_{1} \mathrm{~T}_{\mathrm{F}}$ treated with 100 $\mu \mathrm{M}$ 3TC. bottom right: Retrotransposition efficiency assessed by flow cytometry, relative to $\mathrm{L} 1 \mathrm{~T}_{\mathrm{F}}$. Histogram depicts the mean and standard deviation of three independent biological replicates (black dots) consisting of three technical replicates each. c, CpG methylation ascertained by ONT sequencing of MEFs and a representative miPSC line untreated with lamivudine (CTRL 2). Results are shown for the whole genome (10kbp windows), the proximal promoters $(-1000,+500)$ of protein-coding genes ${ }^{110}$, the $5^{\prime} \mathrm{UTR}$ of $\mathrm{T}_{\mathrm{F}}, \mathrm{G}_{\mathrm{F}}$, and A-type L1s $>6 \mathrm{kbp}, \mathrm{B} 1$ and B2 SINEs, and MERVL MT2 and IAP long terminal repeats. d, Composite L1 $\mathrm{T}_{\mathrm{F}}$ methylation profiles. Each graph displays 100 profiles. A schematic of the $\mathrm{T}_{\mathrm{F}}$ consensus is provided at top. Average values are indicated by more thickly colored lines. e, Methylation profile of the $F s d 1 l$ locus obtained by ONT sequencing. The first panel shows an L1 $\mathrm{T}_{\mathrm{F}}$ orientated in sense to intron 6 of $F s d 1 l$, as well as an expressed sequence tag (EST) obtained from a mouse ESC sample and supporting a transcript initiated in the $\mathrm{T}_{\mathrm{F}}$ 5'UTR and spliced into a downstream Fs 11 exon. The second panel displays ONT read alignments, with unmethylated CpGs colored in brown (MEF) and green (miPSC), methylated CpGs colored black, and CpGs not confidently called, i.e. abs(log-likelihood ratio) $>2.5$, omitted. The third panel indicates the relationship between $\mathrm{CpG}$ positions in genome space and $\mathrm{CpG}$ space, including those corresponding to the $\mathrm{T}_{\mathrm{F}} 5^{\prime} \mathrm{UTR}$ (shaded light blue). The fourth panel indicates the fraction of methylated CpGs. Note: this $\mathrm{L} 1 \mathrm{~T}_{\mathrm{F}}$ is polymorphic in mouse strains ${ }^{55}$. 
(Extended Data Fig. 7d) and one in the feeder MEFs, a potential experimental contaminant (Extended Data Fig. 7d). The remaining 14 putative de novo events comprised 3 L1 TF, 2 L1 GF, 6 B2, and 3 ERV insertions. Although they could not be PCR amplified in any sample, all of the L1 and B2 insertions carried clear TPRT hallmarks (Supplementary Table 3). Each ERV incorporated two long terminal repeats flanking an internal proviral sequence and generated TSDs of the expected size (6bp) ${ }^{79,80}$ (Supplementary Table 3). Although not statistically significant, we found fewer de novo L1-mediated insertions on average in the lamivudine treated miPSCs $(\sim 2)$ than the control miPSCs ( 4), consistent with L1 inhibition by 3TC (Fig. 3b). Overall, ONT sequencing detected endogenous retrotransposition events in bulk miPSCs, providing results orthogonal and complementary to our short-read genomic analyses.

\section{Genome-wide DNA demethylation during reprogramming focused on young L1 loci}

A major feature of reprogramming mouse fibroblasts to a pluripotent state is globally reduced DNA methylation ${ }^{43,44,47}$. Although bisulfite sequencing can estimate the overall methylation of TE families, it can typically only resolve CpGs close to the termini of individual full-length L1 copies not located in highly repetitive regions. To generate a comprehensive genome-wide view of DNA methylation changes during reprogramming, and complement our bisulfite sequencing data, we analyzed the ONT data from MEFs and one of the matched miPSC lines not treated with 3TC, using Methylartist ${ }^{35,81}$. While methylation was reduced genome-wide, on protein-coding gene promoters (Extended Data Fig. 8a), and amongst all of the TE families considered (Extended Data Fig. 8b), the very youngest L1 subfamilies ( $\mathrm{T}_{\mathrm{FI}}$ and $\mathrm{T}_{\mathrm{FII}}$ ) displayed by far the greatest median methylation change (-68.7\%) (Fig. 3c and Supplementary Table 5). 92.1\% of full-length L1 TFI and TFII copies were significantly ( $\mathrm{p}<0.01$, Fisher's exact test with Bonferroni correction) less methylated in miPSCs (Supplementary Table 5), with this demethylation most pronounced in the monomeric L1 5'UTR (Fig. 3d). Thirty-six L1s initiated transcription of a spliced mRNA from their 5'UTR, as defined by GenBank expressed sequence tags, including alternative promoters for protein-coding genes expressed in pluripotent cells, such as Fsdll (Fig. 3e and Supplementary Table 5). We also identified full-length L1s demethylated in both MEFs and miPSCs (Extended

Data Fig. 8c), in line with prior human data suggesting certain L1 loci evade DNA methylation in differentiated cells ${ }^{34,82}$. In sum, ONT analysis showed global reprogramming-associated demethylation is most accentuated for the youngest L1s, where retrotransposition potential is 
280

281

282

283

284

285

286

287

288

289

290

291

292

293

294

295

296

297

298

299

300

301

302

303

304

305

306

307

308

309

310

concentrated, creating opportunities for L1-driven mobilization and protein-coding gene alternative promoters.

\section{Discussion}

This study demonstrates miPSCs incompletely silence mobile TE families and routinely harbor $d e$ novo TE insertions. While some TE insertions occur in parental cells and are inherited by miPSCs, our data suggest the majority arise during reprogramming or very early upon reaching pluripotency. In support of this view, firstly, we observed profound hypomethylation of young L1 promoters in miPSCs and not parental cells. As shown elsewhere, L1 mRNA abundance is low in fibroblasts and increases greatly upon reprogramming $45,46,51$, while engineered L1 reporter genes retrotranspose $>10$-fold more frequently in hiPSCs and hESCs than in fibroblasts ${ }^{50,51}$. Secondly, 38/41 de novo TE insertions detected by Illumina sequencing PCR validated in only one miPSC line each. These and the 14 putative de novo TE insertions identified by ONT sequencing were absent from all other samples in the study, as assayed by PCR and deep WGS. Finally, private SNVs ( 100 per line) and SVs ( 1 per line) were detected at similar frequencies in heterogeneous (bulk) and homogenous (single-cell clone) miPSC populations, whereas far more de novo retrotransposition events were found in the latter experiment. One explanation for this result is that a relatively small number of clones dominate bulk reprogramming experiments ${ }^{83}$ and most SNVs and SVs predate reprogramming ${ }^{10,11,13}$, while retrotransposons mainly mobilize during reprogramming. This model is consistent with a prior WGS analysis that, alongside thousands of SNVs, identified no somatic L1 insertions among 10 human fibroblast clones generated from single cells ${ }^{84}$.

Previous experiments employing hiPSCs and mouse and human ESCs showed L1 derepression and mobilization were likely to take place in pluripotent cells ${ }^{34,41,42,45,46,49-51,85}$. Notably, 23/35 (65.7\%) de novo L1 insertions found here in miPSCs were full-length, a similar percentage to that observed previously in hiPSCs $(57.1 \%)^{45}$. New full-length L1 insertions have potential for further retrotransposition and were largely unmethylated in miPSCs. Their CpG dinucleotides presented a "sloping shore" of methylation, as found elsewhere for newly retrotransposed CpG islands ${ }^{34,35,86}$, where methylation decreases from the L1 5' genome junction and forms a trough before sharply increasing over the L1 ORFs. Only one insertion corresponded to the L1 A subfamily, while the remainder were $\mathrm{T}_{\mathrm{F}}$ and $\mathrm{G}_{\mathrm{F}}$ elements, consistent with relative activity levels 
311

312

313

314

315

316

317

318

319

320

321

322

323

324

325

326

327

328

329

330

331

332

333

334

335

336

337

338

339

340

341

revealed by sequencing extended mouse pedigrees and mouse tumors ${ }^{28,60}$. De novo SINE B1 and B2 insertions, mediated in trans by the L1 protein machinery ${ }^{87}$, were also detected in miPSCs, in line with L1-mediated Alu SINE insertions arising in hiPSCs and hESCs ${ }^{45,85,88}$. Discovery of $d e$ novo TE insertions in low-passage miPSCs derived from multiple parental cell types suggests endogenous retrotransposition may be an intrinsic risk of the epigenome remodeling required for the acquisition of pluripotency $y^{7,43-45,47}$. Retrotransposon insertions into protein-coding genes, such as Brcal and Dmd, could undermine miPSC models of human disease. Such mutations necessitate screening of miPSC lines ${ }^{4}$. However, strategies to minimize TE-mediated mutagenesis, including via the use of 3TC or another L1 reverse transcriptase inhibitor, appear achievable without affecting reprogramming efficiency, and therefore may be incorporated into future miPSC derivation protocols.

\section{Methods}

\section{Ethics statement}

All animal experimentation was performed under the auspices and approval of the Monash University Animal Research Platform Animal Ethics Committee (Approval Numbers MARP2011-172-Polo, MARP-2011-171-BC-Polo, MARP-2017-151-BC-Polo, and ERM\# 21634).

\section{Adult Oct4GFP-OKSM-M2rtTA mouse tissue somatic cell isolation and reprogramming}

Induced pluripotent stem cells were generated from adult and embryonic Oct4GFP-OKSMM2rtTA doxycycline inducible reprogrammable mice ${ }^{52}$. These animals are heterozygous for an Oct4-GFP reporter and an OKSM cassette targeted to the Collagen 1 1 locus, and homozygous for the ROSA26-M2rtTA allele from the ubiquitous ROSA26 locus. The polycistronic cassette is under the control of a tetracycline-dependent promoter (tetOP). Hence, upon the addition of doxycycline, M2rtTA binds to the tetOP, thereby inducing OKSM expression. Oct4GFP-OKSM-M2rtTA mice were housed at the Monash University Animal Research Platform animal facility.

Bone marrow extraction and FACS purification of granulocytes and hematopoietic stem (LSK) cells were performed as previously described ${ }^{89}$. In brief, harvested bone marrow cells were labeled using a two-step sequential antibody labeling procedure using the following primary conjugated antibodies: 1:200 dilution of Anti-Mouse CD5 FITC antibody (BD Biosciences, Cat\#: 553020), 1:100 dilution of Anti-Mouse B220 FITC antibody (BD Biosciences, Cat\#: 557669), 
1:200 dilution of Anti-Mouse TER-119 FITC antibody (BD Biosciences, Cat\#: 557915), 1:400 dilution of Anti-Mouse Sca-1 PB antibody (Biolegend, Cat\#: E13-161.7), 1:200 dilution of AntiMouse cKit APC antibody (BD Biosciences, Cat\#: 553356), 1:200 dilution of Anti-Mouse SSEA1 Biotinylated antibody (Thermo Fisher Scientific, Cat\#: 13-8813-80), 1:200 dilution of Anti-Mouse Gr-1 APC-Cy7 antibody (Biolegend, Cat\#: 108423) and 1:1000 dilution of Anti-Mouse Mac1 PE antibody (Biolegend, Cat\#: 101207). This was followed by the secondary labeling step with 1:200 dilution of Streptavidin PE-Cy7 antibody (BD Biosciences, Cat\#: 557598). Cells were isolated and sorted using an Influx Cell Sorter Instrument (BD Biosciences) with a 100 $\mu \mathrm{m}$ nozzle. Samples were resuspended in phosphate buffered saline (PBS) supplemented with $2 \%$ fetal bovine serum (Thermo Fisher Scientific, Cat\#: SH30071.03FBS, Hyclone). FACS sorting for these and the cell types below were performed with $2 \mu \mathrm{g} / \mathrm{mL}$ Propidium Iodide (PI) (Sigma Aldrich, Cat\#: P4864) in order to exclude non-viable cells. Granulocytes were isolated using the following cell surface marker profile: $\mathrm{CD}^{\circ} / \mathrm{B} 220^{-} / \mathrm{Ter} 119^{-} / \mathrm{Sca} \% \mathrm{cKit}^{-} / \mathrm{SSEA} 1^{\circ} / \mathrm{Gr}^{+} / \mathrm{Mac}^{+}$, whilst $\mathrm{LSK}$ cells were isolated from bone marrow using the following cell surface marker profile: $\mathrm{CD}^{-}{ }^{-} / \mathrm{B} 220^{-} / \mathrm{Ter}^{-} 1^{-}$ $/ \mathrm{Sca}^{+} / \mathrm{cKit}^{+} / \mathrm{SSEA} 1^{-} / \mathrm{Gr}^{-} / \mathrm{Mac}^{-}$.

Fibroblasts were isolated from both ear lobes from each mouse. Tissue pieces were resuspended in 0.25\% Trypsin-EDTA (Thermo Fisher Scientific, Cat\#: 25200-072) solution, and after $5 \mathrm{~min}$ incubation at room temperature, were mechanically minced using two surgical blades for a further $2 \mathrm{~min}$. iPSC medium was used to inactivate trypsin, and dissociated pieces were transferred to a $15 \mathrm{~mL}$ centrifuge tube (Corning). Tissue pieces were then transferred to a gelatin coated T-75 flask (Corning) and cells were left to grow for a further 7 days. CD45 $/ \mathrm{CD}^{-} 1^{-}$ /Thy $1.2^{\text {hi+ }}$ fibroblasts were fractionated by FACs using the following antibodies: a 1:100 dilution of Anti-Mouse CD31 antibody conjugated to FITC (Thermo Fisher Scientific, Cat\#: 11-0311-81), a 1:100 dilution of Anti-Mouse CD45 antibody conjugated to FITC (Thermo Fisher Scientific, Cat\#: 11-0451-810) and a 1:400 dilution of Anti-Mouse Thy-1.2 antibody conjugated to APC (Thermo Fisher Scientific, Cat\#: 17-0902-81).

Liver epithelial cells were isolated according to an adaptation of a previously described method $^{90}$. Briefly, 3mg/mL Collagenase Type 1 (Sigma-Aldrich, Cat\#: C1639) solution was prepared in sterile PBS. Whole liver was transferred into a sterile $6 \mathrm{~cm}$ petri dish and finely minced using fine dissecting scissors. Minced liver pieces were transferred to $15 \mathrm{~mL}$ tube with preheated Collagenase Type 1 (Sigma, Cat\#: C1639). Tubes were left to agitate on a Thermomix (Eppendorf) 
at $750 \mathrm{rpm}, 37^{\circ} \mathrm{C}$ for $15 \mathrm{~min}$. Following digestion, the tube was removed and the cellular suspension was triturated with an $18 \mathrm{G}$ needle, until tissue chunks were mostly dissociated. Sample tubes were then left to agitate for an additional 15min, until liver fragments were completely digested. The sample suspension was again triturated, with a $21 \mathrm{G}$ needle, to generate a single cell suspension, and then processed through a $40 \mu \mathrm{m}$ cell strainer into a clean $50 \mathrm{~mL}$ centrifuge tube (Corning). After rinsing in $2 \% \mathrm{FCS} / \mathrm{PBS}$ (wash buffer) and centrifuging for $5 \mathrm{~min}$ at $1380 \mathrm{rpm}$ for $4^{\circ} \mathrm{C}$, the supernatant was removed and cells were resuspended in wash buffer and centrifuged once again. Cells were counted and $5 \times 10^{6}$ cells were resuspended for sorting. Cells were labeled with primary antibodies using a 1:100 dilution of Anti-mouse CD31 antibody conjugated to FITC (Thermo Fisher Scientific, Cat\#: 11-0311-81), followed by a 1:100 dilution of Anti-mouse CD45 antibody conjugated to FITC (Thermo Fisher Scientific, Cat\#: 11-0451-81) and 1:100 dilution of Antimouse EpCAM antibody conjugated to eFluor450 (Thermo Fisher Scientific, Cat\#: 48-5791-82). Liver epithelial cells were isolated using the following cell surface marker profile: $\mathrm{CD}^{-} 5^{-} / \mathrm{CD} 31^{-}$ $/ \mathrm{EpCAM}^{+ \text {hi }}$.

Thymus tissue was processed for thymic epithelial cell isolation as previously described ${ }^{91}$. Cells were labeled with the following antibodies: 1:400 dilution of Anti-mouse CD45 antibody conjugated to APC-Cy7 (BD Biosciences, Cat\#: 557659), 1:200 dilution of Anti-mouse TER-119 antibody conjugated to APC-Cy7 (BD Biosciences, Cat\#: 560509), 1:6000 dilution of Anti-mouse MHC Class II antibody conjugated to PB (Biolegend, Cat\#: 107620) and 1:1000 dilution of Antimouse EpCAM antibody conjugated to APC (Biolegend, Cat\#: 118214). Thymic epithelial cells were sorted according to the following cell surface marker profile: CD45/Ter119//MHC Class $\mathrm{II}^{+} / \mathrm{EpCAM}^{+}$.

Intestinal stem cells were purified as previously described ${ }^{92}$. Cells were labeled with a 1:200 dilution of Anti-mouse CD45 antibody conjugated to BV510 (BD Biosciences, Cat\#: 563891), 1:200 dilution of Anti-mouse CD31 antibody conjugated to BV510 (BD Biosciences, Cat\#: 563089), a 1:100 dilution of Anti-mouse CD24 antibody conjugated to Pe-Cy7 (Thermo Fisher Scientific, Cat\#: 25-0242-82), a 1:100 dilution of Anti-mouse EpCAM antibody conjugated to eFluor450 (Thermo Fisher Scientific, Cat\#: 48-5791-82), and 1:100 Anti-EphrinB2 unconjugated antibody (BD Biosciences, Cat\#: 743763). In the secondary labeling step, a 1:200 dilution of Anti-mouse Alexa Fluor 555 polyclonal antibody (Thermo Fisher Scientific, Cat\#: A- 
31570) was used to detect the EphrinB2 antibody. Intestinal stem cells were fractionated according to the following cell surface marker profile: CD45/CD31\% $/ \mathrm{CD} 24^{+} / \mathrm{EpCAM}^{+} / \mathrm{Ephrin}^{+}$.

To obtain astrocytes, brain tissue was processed using a MACS Neural Tissue Dissociation Kit (T) (Miltenyi Biotec, Cat\#: 130-093-231) and manually dissected according to manufacturer's instructions. Cells were then collected and incubated with antibodies directed against Glast1 (Allophycocyanin-conjugated, ACSA-1, 1:10 dilution) (Miltenyi Biotec, Cat\#: 130-098-803), 1:100 dilution of Anti-mouse CD133 antibody conjugated to PE (Thermo Fisher Scientific, Cat\#: 12-1331-80), 1:200 dilution of Anti-mouse CD45 antibody conjugated to PE-Cy7 (BD Biosciences, Cat\#: 552848) and 1:200 dilution of Anti-mouse CD31 antibody conjugated to PECy7 (Thermo Fisher Scientific, Cat\#: 25-0311-82). Astrocytes were sorted and purified according to the following cell surface marker profile: CD45\%CD31\%CD133\%GLAST1 ${ }^{+}$.

Keratinocytes and bulge stem cells were isolated from epidermis as previously described ${ }^{93}$. Cells were collected and incubated with antibodies against Anti-Mouse Integrin alpha 6 antibody (GoH3) conjugated to PE (1:600) (Abcam, Cat\#: ab95703), a 1:200 CD104 antibody conjugated to FITC (Biolegend, Cat\#: 346-11A) and a 1:100 dilution of Anti-mouse CD34 biotinylated antibody (Thermo Fisher Scientific, Cat\#: 13-0341-85) for $20 \mathrm{~min}$ at $4^{\circ} \mathrm{C}$. For secondary antibody labeling, cells were incubated with 1:200 APC-Streptavidin antibody (Biolegend, Cat\#: 405207) to detect CD34 biotinylated antibody for $20 \mathrm{~min}$ at $4{ }^{\circ} \mathrm{C}$. They were then washed and resuspended

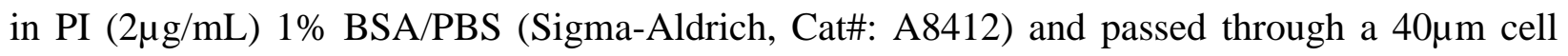
strainer (BD Falcon) to produce single cell suspensions. Cells with the surface marker profile of

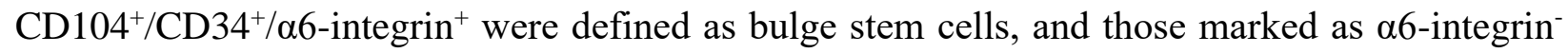
$/ \mathrm{CD} 34^{+}$were defined as keratinocytes.

Reprogramming of the above 9 primary cell types was performed as follows: cells were seeded into gelatinized tissue culture treated 6-well plates (Corning Costar, Cat\#: CLS3506) and cultured at $37^{\circ} \mathrm{C}$ and $5 \% \mathrm{CO}_{2}$ in iPSC media containing KnockOut DMEM (Thermo Fisher Scientific, Cat\#: 10829-018), 15\% Fetal Bovine Serum (FBS) (Thermo Fisher Scientific, Cat\#: SH30071.03), GlutaMAX Supplement (Thermo Fisher Scientific, Cat\#: 35050061), PenicillinStreptomycin (Thermo Fisher Scientific, Cat\#: 15070063), MEM Non-Essential Amino Acids Solution (Thermo Fisher Scientific, Cat\#: 11140050), 2-Mercaptoethanol (Thermo Fisher Scientific, Cat\#: 21985023) and 1000U/mL Leukemia Inhibitory Factor (LIF) (Merck Millipore, Cat\#: ESG1107), supplemented with $2 \mu \mathrm{g} / \mathrm{mL}$ of doxycycline (dox) (Sigma-Aldrich, Cat\#: 33429- 
100MG-R). iPSC medium supplemented with dox was replaced every alternate day after the first 3 days of reprogramming and withdrawn 4 days after the presence of iPSC-like colonies had formed, with typical dome-shaped iPSC morphology. Cells were then cultured to confluency on a layer of irradiated MEFs prior to further FACs purification and enrichment for Oct-GFP ${ }^{+}$cells.

438 Purified Oct4-GFP iPSCs were then bulk expanded in $175 \mathrm{~cm}^{2}$ cell culture flasks (Corning, Cat\#: CLS430825) and then frozen at a density of $1 \times 10^{6}$ cells/vial.

\section{Mouse embryonic fibroblast isolation and reprogramming}

442 Reprogrammable mouse embryonic fibroblast (MEF) cultures were derived as described previously $^{94}$ from a E13.5dpc Oct4GFP-OKSM-M2rtTA embryo (animal I222e2) and cultivated at $37^{\circ} \mathrm{C}, 5 \% \mathrm{O}_{2}, 5 \% \mathrm{CO}_{2}$ in MEF medium containing DMEM High Glucose (Thermo Fisher Scientific, Cat\# 11960-044) with 10\% FBS (Thermo Fisher Scientific, Cat\#: SH30071.03), 1mM Sodium Pyruvate (Thermo Fisher Scientific, Cat\#: 11360-070), GlutaMAX Supplement (Thermo 447 Fisher Scientific, Cat\#: 35050061), Penicillin-Streptomycin (Thermo Fisher Scientific, Cat\#: 15070063), MEM Non-Essential Amino Acids Solution (Thermo Fisher Scientific, Cat\#: 11140050) and 2-Mercaptoethanol (Thermo Fisher Scientific, Cat\#: 21985023). MEFs were reprogrammed by being placed in iPSC medium supplemented with $2 \mu \mathrm{g} / \mathrm{mL}$ dox (Sigma-Aldrich, Cat\#: 33429-100MG-R) and cultured on irradiated MEFs at $37^{\circ} \mathrm{C}, 5 \% \mathrm{CO}_{2}$. iPSC colonies were discerned according to GFP expression in the absence of dox. In addition to bulk iPSC cultures

453 (see below), single Oct4-GFP ${ }^{+}$cells were deposited via FACS individually into 96-well pregelatinized tissue culture plates (Falcon, Cat\#: 353072). Eighteen single-cell clones were bulk expanded on 6-well pre-gelatinized tissue culture plates (Falcon, Cat\#: 353046) and maintained in serum or $2 \mathrm{i}$ conditions (see below).

\section{Oct4-GFP+ ${ }^{+}$PSC flow cytometry}

462 forward scatter, side scatter and PI exclusion. Flow cytometric gates were set using control iPSCs 463 that did not have endogenous GFP expression. Tubes were sorted according to GFP expression 
465

466

467

468

469

470

471

472

473

474

475

476

477

478

479

480

481

482

483

484

485

486

487

488

489

490

491

492

493

494

495

presented using FlowJo software. Sorted $\mathrm{GFP}^{+}$cells were then plated down on T-25 flasks (Corning) and expanded onto T-150 flasks (Corning), before being frozen down at a density of $1 \times 10^{6}$ cells/vial.

\section{Serum and serum-free iPSC culture}

Mouse iPSCs were maintained on irradiated primary MEFs, as previously described ${ }^{8,95}$. Briefly, iPSCs were cultured on 0.2\% Porcine Gelatin (Sigma-Aldrich, Cat\#: G1890-500G) coated tissue culture plates and flasks (Corning) on a feeder layer of irradiated MEFs $\left(2 \times 10^{4} \mathrm{cells} / \mathrm{cm}^{2}\right)$. iPSC medium was changed daily and cells were cultured at $37^{\circ} \mathrm{C}$ and $5 \% \mathrm{CO}_{2}$. Passaging was performed when iPSCs reached 70\% confluency. Alternatively, iPSCs were cultured on irradiated MEFs in serum-free media containing knockout serum replacement (KOSR) and 2i/LIF ${ }^{96}$. Here, cells were cultured in DMEM (Thermo Fisher Scientific, Cat\#: 11960-044), 1000U/mL LIF (Merck Millipore, Cat\#: ESG1107), 0.1mM 2-Mercaptoethanol (Thermo Fisher Scientific, Cat\#: 21985023), 1mM GlutaMAX Supplement (Thermo Fisher Scientific, Cat\#: 35050061), 1\% Sodium Pyruvate (Thermo Fisher Scientific, Cat\#: 11360-070), 0.1mM MEM Non-Essential Amino Acids Solution (Thermo Fisher Scientific, Cat\#: 11140050), 1\% Penicillin-Streptomycin (Thermo Fisher Scientific, Cat\#: 15070063), with medium supplemented with 15\% KOSR (Thermo Fisher Scientific, Cat\#: 10828-028), 1 $\mu \mathrm{m}$ Mek1/2 Inhibitor (PD0325901) (Tocris, Cat\#:

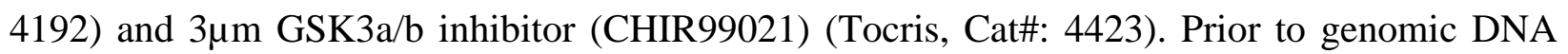
extraction, iPSCs depleted from irradiated feeders were dissociated with $0.5 \%$ Trypsin EDTA (Thermo Fisher Scientific, Cat\#: 25200-072). The irradiated MEFs were feeder depleted with $10 \mathrm{~mL}$ of iPSC media for 45min in non-gelatinized T-25 flasks (Corning, Cat\#: CLS3056). The resultant iPSCs were collected as a supernatant in suspension medium.

\section{Lamivudine titration experiments}

iPSCs were cultured with primary irradiated MEFs, as above, for 9 days in concentrations of lamivudine (3TC, Sigma-Aldrich, Cat\#: L1295-10MG) ranging from 0 to $200 \mu \mathrm{M}$ and cell survival calculated as a \% of the starting population. Reprogrammable OKSM, rtTA3 MEFs were isolated from embryonic day 13.5 embryos from Oct4-GFP;ROSA-rtTA-out;OKSM-72 mice as previously described $^{44,97}$. Doxycycline inducible reprogrammable MEFs were grown in media containing $2 \mu \mathrm{g} / \mathrm{mL}$ dox (Sigma Aldrich Cat\#: 33429-100MG-R) and 0-200 $\mu \mathrm{M}$ 3TC for 15 days, with the 
496

497

498

499

500

501

502

503

504

505

506

507

508

509

510

511

512

513

514

515

516

517

518

519

520

521

522

523

524

525

526

percentage cell survival calculated at days 3, 7, 10 and 15 . Once $100 \mu \mathrm{M}$ was identified as the optimal concentration of 3TC to assess its impact on L1 retrotransposition, 30,000 reprogrammable MEFs at passage 2 were seeded onto gelatinized 6-well plates and reprogrammed in dox for 12 days, then cultured for an additional 4 days without dox. Oct4-GFP ${ }^{+}$iPSCs were then purified via flow cytometric sorting and expanded on irradiated MEFs for an additional 11 days, then feeder depleted prior to DNA extraction. Reprogramming and iPSC media contained serum, and either $100 \mu \mathrm{M} 3 \mathrm{TC}$ or no $3 \mathrm{TC}$.

\section{Illumina sequencing and genomic analysis}

Genomic DNA was harvested from MEFs and iPSCs using a DNeasy Blood and Tissue Kit (Qiagen, Cat\#: 60594). DNA was quantified by a Qubit dsDNA HS Assay Kit (Life Technologies, Cat\#: Q32851) on a Qubit Fluorometer 3.0 (Life Technologies). For WGS, libraries were generated using an Illumina TruSeq DNA PCR-free kit (Illumina, Cat\#: 20015962) and sequenced separately on an Illumina HiSeq X Ten platform (Macrogen, Korea).

For mRC-seq, libraries were prepared as follows: $1 \mu \mathrm{g}$ genomic DNA was sheared using a Covaris M220 Focused Ultrasonicator in a $130 \mu \mathrm{L}$ microTUBE AFA fiber snap-cap vial (Covaris, Cat\#: 520045). The following parameters were used to gain 500bp insert libraries: 50W, duty factor 20\%, 200 cycles per burst, duration 55s. Size selection to remove fragments $<300 \mathrm{bp}$ was performed using Agencourt AMPure XP beads (Beckman Coulter, Cat\#: A63881) with a 1:0.6 DNA:beads ratio. Libraries were then generated by TruSeq Nano DNA LT kit (Illumina, Cat\#: 20015964) using TruSeq DNA Single Indexes (Illumina, Cat\#: 20015960 and 20015961) and run on a $2 \%$ agarose gel (Bioline, Cat\#: BIO-41025) pre-stained with SYBR Safe Nucleic Acid Gel Stain (Invitrogen, Cat\#: S33102). For 500bp insert size libraries the target gel fragment size was 600-650bp, which was excised under a Safe Imager 2.0 Blue-Light Transilluminator (Invitrogen). DNA was purified using a MinElute Gel Extraction Kit (Qiagen, Cat\#: 28606) according to the manufacturer's instructions. DNA was eluted in $25 \mu \mathrm{L}$ molecular grade water. Enrichment of DNA fragments was performed as described for Illumina TruSeq Nano DNA LT Kit (Illumina, Cat\#: 20015964). Sample clean up was performed with Agencourt AMPure XP beads (Beckman Coulter, Cat\#: A63881) using a 1:1.1 ratio of DNA to beads. Amplified libraries were eluted in $30 \mu \mathrm{L}$ molecular grade water and quantified using a Bioanalyzer DNA 1000 chip (Agilent Technologies, Cat\#: 5067-1504). 
mRC-seq hybridization was performed as previously described ${ }^{28}$. Hybridization reactions were washed using SeqCap Hybridization and Wash Kit (Roche, Cat\#: 05634261001) and DNA eluted in $50 \mu \mathrm{L}$ molecular grade water. Two post-hybridization LM-PCR reactions per sample were performed using $20 \mu \mathrm{L}$ Enhanced PCR Mix, $5 \mu \mathrm{L}$ PCR Primer Cocktail from the Illumina TruSeq

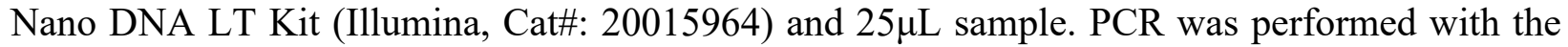
following cycling conditions: $95^{\circ} \mathrm{C}$ for $3 \mathrm{~min}, 8$ cycles of $98^{\circ} \mathrm{C}$ for $20 \mathrm{~s}, 60^{\circ} \mathrm{C}$ for $15 \mathrm{~s}$, and $72^{\circ} \mathrm{C}$ for 30s, followed by $72^{\circ} \mathrm{C}$ for $5 \mathrm{~min}$. The two PCR reactions for each sample were pooled and cleaned up using the QIAquick PCR Purification Kit (Qiagen) and samples eluted in $15 \mu \mathrm{L}$ Elution Buffer (Qiagen, Cat\#: 28706). Quantity and fragment size were determined using a Bioanalyzer DNA 1000 chip (Agilent Technologies, Cat\#: 5067-1504). Libraries were pooled and sequenced on an Illumina HiSeq X Ten platform (Macrogen, Korea).

Reads were aligned to the mm10 reference genome using bwa-mem ${ }^{98}$ version 0.7 .12 with parameters -M -Y. Duplicate reads were marked via Picard MarkDuplicates version 1.128. Indel Realignment was carried out via GATK IndelRealigner (3.7). SNVs were called by GATK HaplotypeCaller $3.7^{53}$ to generate GVCFs and GenotypeGVCFs to obtain cohort-level calls. SNVs were also called using freebayes ${ }^{54}$ filtered to remove known mouse strain germline variants ${ }^{55}$. SVs were called using Delly2 and GRIDSS 2.0.0 $0^{56,57}$, using calls with concordant non-filtered precise breakends. Variant impact prediction and annotation was carried out using SnpEff version 4.3T ${ }^{99}$. WGS and mRC-seq aligned BAMs were processed to identify non-reference TE insertions using TEBreak (https://github.com/adamewing/tebreak) as previously described ${ }^{60}$.

\section{TE insertion PCR validation experiments}

Reads supporting putative de novo TE insertions were manually examined using Serial Cloner (http://serialbasics.free.fr/Serial_Cloner.html), the UCSC Genome Browser BLAT tool ${ }^{100}$ and the Repbase CENSOR tool ${ }^{101}$. PCR primers were designed with Primer $3^{102}$ against TE insertion sequences and their 5' and 3' genomic flanks (Supplementary Table 3). Empty/filled PCRs (combining 5' and 3' flanking primers) and full-length PCRs (using junction-spanning primers) were performed using an Expand Long Range dNTPack (Roche, Cat\#: 4829034001). Reaction mixes contained $5 \mu \mathrm{L} 5 \times$ Expand Long Range Buffer with $12.5 \mathrm{mM} \mathrm{MgCl} 2,1.25 \mu \mathrm{L}$ dNTP Mix (dATP, dCTP, dGTP, dTTP at 10mM each), 1.25 $\mu \mathrm{L}$ DMSO (100\%), 1 $\mu \mathrm{L}$ primer mix $(25 \mu \mathrm{M}$ of each primer), $0.35 \mu \mathrm{L}$ Expand Long Range Enzyme Mix (5U/ $\mu \mathrm{L}), 4-10$ ng genomic DNA template, 
and molecular grade water up to a total volume of $25 \mu \mathrm{L}$. PCR was performed with the following cycling conditions: $92^{\circ} \mathrm{C}$ for $3 \mathrm{~min}, 10$ cycles of $92^{\circ} \mathrm{C}$ for $30 \mathrm{~s}, 56-60^{\circ} \mathrm{C}$ for $30 \mathrm{~s}$, and $68^{\circ} \mathrm{C}$ for $7 \mathrm{~min}$ $30 \mathrm{~s} 25$ cycles of $92^{\circ} \mathrm{C}$ for $30 \mathrm{~s}, 56-60^{\circ} \mathrm{C}$ for $30 \mathrm{~s}$, and $68^{\circ} \mathrm{C}$ for $7 \mathrm{~min}+20 \mathrm{~s}$ cycle elongation for each successive cycle, followed by $68^{\circ} \mathrm{C}$ for $10 \mathrm{~min}$. TE-genome junction validation PCRs were performed using MyTaq HS DNA Polymerase (Bioline, Cat\#: BIO-2111). Reaction mixes contained $5 \mu \mathrm{L} 5 \times$ MyTaq Reaction Buffer, $0.5 \mu \mathrm{L}$ primer mix $(25 \mu \mathrm{M}$ of each primer $), 0.2 \mu \mathrm{L}$ MyTaq HS DNA Polymerase, 2-4ng genomic DNA template, and molecular grade water up to a total volume of $25 \mu \mathrm{L}$. PCRs were performed using the following conditions: $95^{\circ} \mathrm{C}$ for $2 \mathrm{~min}, 35$ cycles of $95^{\circ} \mathrm{C}$ for $15 \mathrm{~s}, 55 / 57^{\circ} \mathrm{C}$ for $15 \mathrm{~s}$, and $72^{\circ} \mathrm{C}$ for $10 \mathrm{~s}$, followed by $72^{\circ} \mathrm{C}$ for $10 \mathrm{~min}$. PCR products were run on 0.8-2\% agarose gels (Bioline, Cat\#: BIO-41025), depending on fragment size, pre-stained with SYBR Safe Nucleic Acid Gel Stain (Invitrogen, Cat\#: S33102). A Typhoon FLA 9000 (GE Healthcare Life Sciences) was used for gel imaging. Gel fragments were excised under a Safe Imager 2.0 Blue-Light Transilluminator (Invitrogen). DNA purification was performed using the QIAquick Gel Extraction Kit (Qiagen, Cat\#: 28706) or MinElute Gel Extraction Kit (Qiagen, Cat\#: 28606) according to the manufacturer's instructions. PCR fragments were either sequenced directly or cloned using the pGEM-T Easy Vector System (Promega, Cat\#: A1360) and Sanger sequenced to resolve insertion characteristics, as shown in Supplementary Table 3.

\section{L1-mCherry retrotransposition assays}

The L1-mCherry construct is derived from the construct pTN201, a pCEP4-based vector containing the native mouse element $\mathrm{L} 1_{\mathrm{spa}}{ }^{22}$. The $\mathrm{L} 1_{\mathrm{spa}}$ coding sequence was modified by sitedirected mutagenesis to include two nonsynonymous nucleotide substitutions, rendering the ORF1p amino acid sequence identical to that of the L1 $\mathrm{T}_{\mathrm{F}}$ subfamily consensus sequence ${ }^{103}$. The 3'UTR is interrupted by a reporter cassette based on previously described L1 retrotransposition indicator plasmids ${ }^{19,104}$. This reporter cassette consists of the mCherry coding sequence in antisense orientation to the L1 and is equipped with an EF1 $\alpha$ promoter and HSVtk polyadenylation signal. The mCherry ORF is interrupted by a $\beta$-globin intron oriented in sense to the L1. The mCherry cassette was cloned using G-block double-stranded DNA fragments synthesized by Integrated DNA Technologies (IDT) and PCR products generated using Q5 DNA polymerase (New England Biolabs, Cat\#: M0492). The mCherry coding sequence was synthesized with silent 
mutations ablating potential splice donor and splice acceptor sites that could interfere with intended splicing of the intron. In the L1-mCherry construct, the final $157 \mathrm{bp}$ of the $\mathrm{L} 1_{\text {spa }} 3^{\prime} \mathrm{UTR}$, which includes a conserved poly-purine tract, are situated downstream of the mCherry cassette and immediately upstream of the pCEP4 SV40 polyadenylation signal. The L1-mCherry_RTmutant contains a missense mutation in the reverse transcriptase domain of ORF2 (D709Y) ${ }^{22}$. Plasmids were prepared using a Qiagen Plasmid Plus Midi Kit and a QIAvac vacuum manifold (Qiagen, Cat\#: 12145).

HeLa-JVM cells ${ }^{19}$ were cultured at $37^{\circ} \mathrm{C}$ and $5 \% \mathrm{CO}_{2}$ in HeLa complete medium (DMEM, Life Technologies, Cat\#: 11960044) supplemented with 10\% FBS (Life Technologies, Cat\#: 10099141), 1\% Glutamax (Life Technologies, Cat\#: 35050061) and 1\% penicillin-streptomycin (Life Technologies, Cat\#: 15140122). Cells were passaged at 70-80\% confluency using 0.25\% Trypsin-EDTA (Life Technologies, Cat\#: 25200072). Cultured cell retrotransposition assays were then performed as described previously ${ }^{66,104}$, except retrotransposition was detected by mCherry fluorescence instead of EGFP fluorescence. Briefly, $1 \times 10^{5} \mathrm{HeLa}-J V M$ cells were seeded per well of a 6-well plate. Eighteen hours later, cells were transfected with $1 \mu \mathrm{g}$ L1-mCherry or L1mCherry_RT-plasmid per well using 3 $\mu \mathrm{L}$ FuGENE HD transfection reagent (Promega, Cat\#: E2311) and 97 $\mu \mathrm{L}$ Opti-MEM (Life Technologies, Cat\#: 31985047) per well according to the manufacturer's protocol. Twenty-four hours post-transfection, medium was replaced with either HeLa complete medium with $200 \mu \mathrm{g} / \mathrm{mL}$ Hygromycin (Life Technologies, Cat\#: 10687010), or HeLa complete medium with $200 \mu \mathrm{g} / \mathrm{mL}$ Hygromycin and $100 \mu \mathrm{M}$ Lamivudine (Sigma-Aldrich, Cat\#: L1295-10MG). Medium was replaced every other day, and at 8 days post-transfection cells were collected by trypsinization, resuspended in sterile PBS, and analyzed on a CytoFLEX flow cytometer (Beckman Coulter) to determine the percentage of mCherry positive cells. Three biological replicate assays were performed, each consisting of 3 assayed wells per condition (technical replicates).

\section{L1-mneoI retrotransposition assays}

To prepare reporter constructs, miPSC_1_L1 and miPSC_4_L1 were amplified from genomic DNA using an Expand Long Range dNTPack (Roche, Cat\#: 4829034001). Reaction mixes contained $5 \mu \mathrm{L} 5 \times$ Expand Long Range Buffer with $12.5 \mathrm{mM} \mathrm{MgCl} 2,1.25 \mu \mathrm{L}$ dNTP Mix (dATP, dCTP, dGTP, dTTP at 10mM each), 1.25 $\mu \mathrm{L}$ DMSO (100\%), $1 \mu \mathrm{L}$ primer mix $(50 \mu \mathrm{M}$ of each 
620

621

622

623

624

625

626

627

628

629

630

631

632

633

634

635

636

637

638

639

640

641

642

643

644

645

646

647

648

649

650

primer), $0.35 \mu \mathrm{L}$ Expand Long Range Enzyme Mix $(5 \mathrm{U} / \mu \mathrm{L})$, 10ng genomic DNA template and molecular grade water, up to a total volume of $25 \mu \mathrm{L}$. PCRs were performed with the following cycling conditions: $92^{\circ} \mathrm{C}$ for $3 \mathrm{~min}, 10$ cycles of $92^{\circ} \mathrm{C}$ for $30 \mathrm{~s}, 58^{\circ} \mathrm{C}$ for $30 \mathrm{~s}$, and $68^{\circ} \mathrm{C}$ for $7 \mathrm{~min} 30 \mathrm{~s}$; 25 cycles of $92^{\circ} \mathrm{C}$ for $30 \mathrm{sec}, 58^{\circ} \mathrm{C}$ for $30 \mathrm{~s}$, and $68^{\circ} \mathrm{C}$ for $7 \mathrm{~min}$ plus 20 s elongation for each successive cycle, followed by $68^{\circ} \mathrm{C}$ for $10 \mathrm{~min}$. Primers introduced a NotI restriction site at the L1 5' end (miPSC_1_L1_F, 5'-tttgcggccgcagaaagggaataatcgaggtg-3'; miPSC_1_L1_R, 5'gctaagcttgagaataagtgaagga-3'; miPSC_4_L1_F, 5'-agggcggccgcaggattaagaacccaatcaccag-3'; miPSC_4_L1_R, 5'-aaaatgcctgttgtgccaat-3'). Reactions were purified using agarose gel electrophoresis. Target fragments were excised and purified using either traditional phenolchloroform extraction or QIAquick and MinElute Gel Extraction Kits (Qiagen, Cat\#: 28706 and 28604). Each L1 was then cloned into pGEMT Easy Vector (Promega, Cat\#: A1360). Ligations were incubated overnight at $4^{\circ} \mathrm{C}$. Ligation reactions were transformed using One Shot TOP10 chemically competent E. coli (Invitrogen, Cat\#: C404010). Blue/white screening was performed using LB/ampicillin/IPTG/X-Gal plates. At least 3 positive colonies per L1 were chosen for Miniprep culture and plasmid DNA was isolated using a QIAprep Spin Miniprep Kit (Qiagen, Cat\#: 27106). At least three clones per element were capillary sequenced and compared to identify PCR-induced mutations. Full-length L1s were then reconstructed by combining PCR-mutation free fragments from different clones using restriction enzymes (New England Biolabs) recognizing the L1 sequence. Reactions were purified using agarose gel electrophoresis and target fragments were excised and purified using QIAquick and MinElute Gel Extraction Kits (Qiagen, Cat\#: 28706 and 28604).

pTN201 was used to generate L1 reporter constructs. pTN201 is composed of a pCEP4 backbone (Life Technologies) containing $\mathrm{L}_{\mathrm{spa}}$, a retrotransposition-competent $\mathrm{L} 1 \mathrm{~T}_{\mathrm{F}}^{22}$ and a downstream mneoI retrotransposition reporter cassette ${ }^{105}$. The mneoI cassette is driven by an SV40 promoter and holds the neomycin resistance gene, which is interrupted by an intron and is positioned antisense to L1spa. In this assay, neomycin (or its analog, Geneticin/G418) resistance only occurs via transcription, splicing and integration of the L1 and mneoI cassette into genomic DNA $^{19,66}$. To measure miPSC_1_L1 and miPSC_4_L1 retrotransposition efficiency, L1 spa was removed from the pCEP4 backbone by digesting with NotI and PacI. The pCEP4 backbone was dephosphorylated using Calf Intestinal Alkaline Phosphatase (CIP) (New England Biolabs, Cat\#: M0290). The backbone and fragments of either miPSC_1_L1 or miPSC_4_L1 were combined in 
651 a single ligation reaction using T4 DNA Ligase (New England Biolabs, Cat\#: M0202) and

652 incubated overnight at $16^{\circ} \mathrm{C}$. Ligations were transformed using One Shot TOP10 chemically competent E. coli (Invitrogen, Cat\#: C404010) and plasmid DNA of positive clones was obtained using QIAprep Spin Miniprep Kit (Qiagen, Cat\#: 27106). Clones were verified as mutation-free by capillary sequencing. Plasmid DNA for retrotransposition assays was obtained using a Plasmid Maxi Kit (Qiagen, Cat\#: 12163). Each construct was built with and without a cytomegalovirus promoter (CMVp) preceding the L1. In addition, the following controls, each based on a pCEP4 backbone containing the mneoI cassette, were employed: TGF21, a retrotransposition-competent L1 $\mathrm{GF}^{21}$; L1SM, a synthetic codon optimized mouse L1 ${ }^{67}$; L1SMmut2, L1SM immobilized by reverse transcriptase and endonuclease domains mutations ${ }^{67}$. modifications. HeLa-JVM cells were grown in HeLa complete medium (DMEM, Life Technologies, Cat\#: 11960044) supplemented with 10\% FBS (Life Technologies, Cat\#: 10099141), 1\% Glutamax (Life Technologies, Cat\#: 35050061) and 1\% penicillin-streptomycin (Life Technologies, Cat\#: 15140122), and then seeded at a density of $4 \times 10^{4}$ cells/well in 6-well tissue culture plates. 14-16h after plating, cells were transfected with L1 reporter constructs using $4 \mu \mathrm{L}$ FuGENE HD transfection reagent (Promega, Cat\#: E2311) 96 $\mu \mathrm{L}$ Opti-MEM (Life

668 Technologies, Cat\#: 31985047) and $1 \mu \mathrm{g}$ plasmid DNA per well. Transfection efficiencies were determined in parallel by preparing transfection mixes containing $4 \mu \mathrm{L}$ FuGENE HD transfection

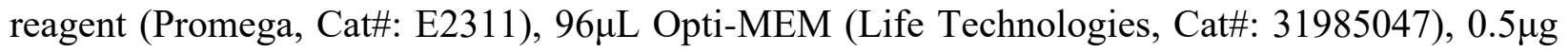

671 L1 expression plasmid and $0.5 \mu \mathrm{g}$ pCEP4-eGFP. The transfection mixture was added to each well 672 containing $2 \mathrm{~mL}$ DMEM-complete medium. Plates were incubated at $37^{\circ} \mathrm{C}$ and $5 \% \mathrm{CO}_{2}$, medium 673 replaced $24 \mathrm{~h}$ post-transfection, and transfection efficiency determined $72 \mathrm{~h}$ post-transfection. pCEP4-eGFP transfected wells were trypsinized and cells were collected from each well and centrifuged at $2000 \mathrm{~g}$ for $5 \mathrm{~min}$. Cell pellets were resuspended in $300-500 \mu \mathrm{L} 1 \times \mathrm{PBS}$. The number 676 of eGFP-positive cells was determined using a CytoFLEX flow cytometer (Beckman Coulter). The 677 percentage of eGFP-positive cells was used to normalize the G418-resistant colony counts for each 678 L1 reporter construct ${ }^{66}$. G418 $(400 \mu \mathrm{g} / \mathrm{mL})$ (Thermo Fisher Scientific, Cat\#: 10131035) selection 679 was started 3 days post-transfection and performed for 12 days. G418 foci were washed with 1× 680 PBS and fixed using 2\% Formaldehyde/0.2\% Glutaraldehyde in 1× PBS (Sigma-Aldrich) fixing 
682 (Sigma-Aldrich) at room temperature for 10min. Foci were counted in each well to quantify retrotransposition.

684

\section{L1 bisulfite sequencing experiments}

686

687

688

689

690

691

692

693

694

695

696

697

698

699

700

701

702

703

704

705

706

707

708

709

710

711

712

Bisulfite conversion was performed with 200ng input genomic DNA from miPSC lines and MEFs using a EZ DNA Methylation-Lightning Kit (Zymo Research, Cat\#: D5030), following the manufacturer's instructions. DNA was eluted in $10 \mu \mathrm{L}$ Elution Buffer. The internal sequences of L1 TF monomers were amplified genome-wide with the following primers: BS_TfIII_mono_F, 5'GGAAATTAGTTTGAATAGGTTAGAGGGTG;

BS_TfIII_mono_R,

$5^{\prime}-$ TCCTAAATTCCAAAAAATCCTAAAACCAAA. The following locus-specific primers were used to target the $5^{\prime}$ promoter region of the following elements of interest: BS_miPSC_1_L1_F, 5'-TGATTTATTTTTGATTGAATTTATTTTTAT; BS_miPSC_1_L1_R/donor_L1_R, 5'-CTATTCAAACTAATTTCCTAAАTTCTACTA; BS_miPSC_3_L1_F, 5'TAGTTGGGGTTGTATGATGTAAGTT; BS_miPSC_3_L1_R, 5'TCCCAAAAACTATCTAATTCTCTAAC; BS_miPSC_4_L1_F, 5'TTTATATTGAAGGTTTGGATGATTTTATAT; BS_miPSC_4_L1_R, 5 TCCAATTCTCTAATACACCCTCTAAC; BS_donor_L1_F $5^{\prime}-$ TTAAAGAAGTTAGTGATTTTTTAGAATTTT.

PCRs were performed using MyTaq HS DNA Polymerase (Bioline, Cat\#: BIO-21111). Reaction mixes contained $5 \mu \mathrm{L} 5 \times$ MyTaq Reaction Buffer, $0.5 \mu \mathrm{L}$ primer mix $(25 \mu \mathrm{M}$ of each primer $), 0.2 \mu \mathrm{L}$ MyTaq HS DNA Polymerase, DMSO at a final concentration of $0.1 \%, 2 \mu \mathrm{L}$ bisulfite converted DNA template, and molecular grade water up to a total volume of $25 \mu \mathrm{L}$. PCR cycling parameters were as follows: $95^{\circ} \mathrm{C}$ for $2 \mathrm{~min}, 40$ cycles of $95^{\circ} \mathrm{C}$ for $30 \mathrm{~s}, 54^{\circ} \mathrm{C}$ for $30 \mathrm{~s}$, and $72^{\circ} \mathrm{C}$ for $30 \mathrm{~s}$, followed by $72^{\circ} \mathrm{C}$ for $5 \mathrm{~min}$. PCR products were run on a $2 \%$ agarose gel, excised and purified using a MinElute Gel Extraction Kit (Qiagen, Cat\#: 28604) according to the manufacturer's instructions. Illumina libraries were constructed using a NEBNext Ultra ${ }^{\mathrm{TM}}$ II DNA Library Prep Kit (New England Biolabs, Cat\#: E7645). Libraries were quantified using a Bioanalyzer DNA 1000 chip (Agilent Technologies, Cat\#: 5067-1504). Barcoded libraries were pooled in equimolar amounts and sequenced as 2x300mer reads on an Illumina MiSeq platform using a MiSeq Reagent Kit v3 (Illumina, Cat\#: MS-102-3003). 50\% PhiX Control v3 (Illumina, Cat\#: FC-110-3001) was used as a spike-in. Sequencing data were analyzed as described previously ${ }^{34}$. To summarize, for the L1 $\mathrm{T}_{\mathrm{F}}$ 
713

714

715

716

717

718

719

720

721

722

723

724

725

726

727

728

729

730

731

732

733

734

735

736

737

738

739

740

741

742

743

genome-wide analysis, paired-end reads were considered separately and those with the L1 $\mathrm{T}_{\mathrm{F}}$ bisulfite PCR primers at their termini were retained and aligned to the mock converted $\mathrm{T}_{\mathrm{F}}$ monomer target amplicon sequence with blastn. Reads where non-CpG cytosine bisulfite conversion was $<95 \%$, or $\geq 5 \%$ of $\mathrm{CpG}$ dinucleotides were mutated, or $\geq 5 \%$ of adenine and guanine nucleotides were mutated, were removed. 50 reads per sample, excluding identical bisulfite sequences, were randomly selected and analyzed using QUMA ${ }^{106}$ with default parameters, with strict CpG recognition. Specific L1 loci were analyzed in a similar fashion, except paired-end reads were assembled into contigs, as described elsewhere ${ }^{34}$, prior to blastn alignment to the mock converted L1 locus target amplicon.

\section{Nanopore sequencing analyses}

Genomic DNA was extracted from 2 miPSC lines reprogrammed without 3TC, 2 miPSC lines generated without 3TC, and the parental MEFs, with a Nanobind CBB Big DNA Kit (Circulomics, Cat\#: NB-900-001-01) according to the manufacturer's instructions. DNA libraries were prepared at the Kinghorn Centre for Clinical Genomics (KCCG, Australia) using $3 \mu$ g input DNA, without shearing, and an SQK-LSK110 ligation sequencing kit. Libraries were each sequenced separately on a PromethION (Oxford Nanopore Technologies) flow cell (FLO-PRO002, R9.4.1 chemistry) (Supplementary Table 1). Bases were called with guppy 5.0.13 (Oxford Nanopore Technologies).

Non-reference TE insertions were detected with TLDR ${ }^{35}$. Briefly, this involved aligning ONT reads to the mm10 reference genome using minimap $2^{107}$ version 2.20 (index parameter: $-\mathrm{X}$ map-ont; alignment parameters: -ax map-ont -L -t 32) and SAMtools ${ }^{108}$ version 1.12. BAM files were then processed as a group with $\mathrm{TLDR}^{35}$ version 1.2.2 (parameters -e teref.mouse.fa -p 128 m 1 -r mm10.fa -n nonref.collection.mm10.chr.bed.gz --keep_pickles). The files teref.mouse.fa, composed of TE family consensus sequences, and nonref.collection.mm10.chr.bed.gz, a collection of known non-reference retrotransposon insertions, are available from github.com/adamewing/tldr/. The TLDR output table was further processed to remove calls not passing relevant TLDR filters, where family $=$ "NA" or remappable = "FALSE" or UnmapCover $<0.5$ or LengthIns $<100$ or EndTE-StartTE $<100$ or strand $=$ "None" or SpanReads $<1.3$ ' truncated TE insertions, and B1 or B2 insertions $5^{\prime}$ truncated by more than $2 \mathrm{bp}$, were removed. Events detected in only one miPSC line and not matching a known non-reference insertion were 
designated as putative de novo insertions (Supplementary Table 3).

Reference TE methylation was assessed for parental MEFs and an miPSC line not treated with 3TC (CTRL 2) with Methylartist version 1.0.6 ${ }^{81}$ Briefly, $\mathrm{CpG}$ methylation calls were generated from ONT reads using nanopolish version 0.13.2 ${ }^{109}$. Using Methylartist commands dbnanopolish, segmeth and segplot with default parameters, methylation statistics were generated for the genome divided into $10 \mathrm{kbp}$ bins, protein-coding gene promoters defined the Eukaryotic Promoter Database $(-1000 \mathrm{bp},+500 \mathrm{bp})^{110}$, and reference TEs defined by RepeatMasker coordinates (http://www.repeatmasker.org/). TE families displayed in Fig. 4c included TF, GF, and A-type L1s $>6 \mathrm{kbp}, \mathrm{B} 1$ and B2 SINEs, and MERVL MT2 and IAP elements represented by their long terminal repeats. Methylation values were calculated for L1 5'UTRs only, excluding the L1 body. Methylation profiles for individual loci were generated using the Methylartist command locus. L1 $\mathrm{T}_{\mathrm{F}}$ methylation profiles shown in Fig. 4d were generated for elements $>7 \mathrm{kbp}$ with the Methylartist command composite. To identify individual differentially methylated TEs (Supplementary Table 5), we required elements to have at least 4 reads and 20 methylation calls in each sample. Statistical comparisons were performed based on methylated and unmethylated $\mathrm{CpG}$ call counts, using Fisher's exact test with Bonferroni correction for multiple testing.

\section{Data availability}

All Oxford Nanopore Technologies and Illumina sequencing data generated by this study were deposited in the European Nucleotide Archive (ENA) under project PRJEB20569.

\section{Code availability}

TEBreak, TLDR and Methylartist, and instructions for their use, are available at https://github.com/adamewing/tebreak, $\quad \underline{\text { https://github.com/adamewing/TLDR } \quad \text { and }}$ https://github.com/adamewing/methylartist, respectively.

\section{References}

1. Takahashi, K. \& Yamanaka, S. Induction of pluripotent stem cells from mouse embryonic and adult fibroblast cultures by defined factors. Cell 126, 663-676 (2006).

2. Mandai, M. et al. Autologous Induced Stem-Cell-Derived Retinal Cells for Macular Degeneration. N. Engl. J. Med. 376, 1038-1046 (2017). 
775

776

777

778

779

780

781

782

783

784

785

786

787

788

789

790

791

792

793

794

795

796

797

798

799

800

801

802

803

804

805

3. Takahashi, K. \& Yamanaka, S. A decade of transcription factor-mediated reprogramming to pluripotency. Nat. Rev. Mol. Cell Biol. 17, 183-193 (2016).

4. Tapia, N. \& Schöler, H. R. Molecular Obstacles to Clinical Translation of iPSCs. Cell Stem Cell 19, 298-309 (2016).

5. Gore, A. et al. Somatic coding mutations in human induced pluripotent stem cells. Nature 471, 63-67 (2011).

6. Hussein, S. M. et al. Copy number variation and selection during reprogramming to pluripotency. Nature 471, 58-62 (2011).

7. Lister, R. et al. Hotspots of aberrant epigenomic reprogramming in human induced pluripotent stem cells. Nature 471, 68-73 (2011).

8. Polo, J. M. et al. Cell type of origin influences the molecular and functional properties of mouse induced pluripotent stem cells. Nat. Biotechnol. 28, 848-855 (2010).

9. Laurent, L. C. et al. Dynamic changes in the copy number of pluripotency and cell proliferation genes in human ESCs and iPSCs during reprogramming and time in culture. Cell Stem Cell 8, 106-118 (2011).

10. Young, M. A. et al. Background mutations in parental cells account for most of the genetic heterogeneity of induced pluripotent stem cells. Cell Stem Cell 10, 570-582 (2012).

11. Cheng, L. et al. Low incidence of DNA sequence variation in human induced pluripotent stem cells generated by nonintegrating plasmid expression. Cell Stem Cell 10, 337-344 (2012).

12. Quinlan, A. R. et al. Genome sequencing of mouse induced pluripotent stem cells reveals retroelement stability and infrequent DNA rearrangement during reprogramming. Cell Stem Cell 9, 366-373 (2011).

13. Abyzov, A. et al. Somatic copy number mosaicism in human skin revealed by induced pluripotent stem cells. Nature 492, 438-442 (2012).

14. Kazazian, H. H., Jr \& Moran, J. V. Mobile DNA in Health and Disease. N. Engl. J. Med. 377, 361-370 (2017).

15. Doucet, A. J., Wilusz, J. E., Miyoshi, T., Liu, Y. \& Moran, J. V. A 3’ Poly(A) Tract Is Required for LINE-1 Retrotransposition. Mol. Cell 60, 728-741 (2015).

16. Jurka, J. Sequence patterns indicate an enzymatic involvement in integration of mammalian retroposons. Proc. Natl. Acad. Sci. U. S. A. 94, 1872-1877 (1997). 
17. Luan, D. D., Korman, M. H., Jakubczak, J. L. \& Eickbush, T. H. Reverse transcription of R2Bm RNA is primed by a nick at the chromosomal target site: a mechanism for non-LTR retrotransposition. Cell 72, 595-605 (1993).

18. Monot, C. et al. The specificity and flexibility of 11 reverse transcription priming at imperfect T-tracts. PLoS Genet. 9, e1003499 (2013).

19. Moran, J. V. et al. High frequency retrotransposition in cultured mammalian cells. Cell 87, 917-927 (1996).

20. Wei, W. et al. Human L1 retrotransposition: cispreference versus trans complementation. Mol. Cell. Biol. 21, 1429-1439 (2001).

21. Goodier, J. L., Ostertag, E. M., Du, K. \& Kazazian, H. H., Jr. A novel active L1 retrotransposon subfamily in the mouse. Genome Res. 11, 1677-1685 (2001).

22. Naas, T. P. et al. An actively retrotransposing, novel subfamily of mouse L1 elements. EMBO J. 17, 590-597 (1998).

23. Nellåker, C. et al. The genomic landscape shaped by selection on transposable elements across 18 mouse strains. Genome Biol. 13, R45 (2012).

24. Skowronski, J., Fanning, T. G. \& Singer, M. F. Unit-length line-1 transcripts in human teratocarcinoma cells. Mol. Cell. Biol. 8, 1385-1397 (1988).

25. Beck, C. R. et al. LINE-1 retrotransposition activity in human genomes. Cell 141, 11591170 (2010).

26. Brouha, B. et al. Hot L1s account for the bulk of retrotransposition in the human population. Proc. Natl. Acad. Sci. U. S. A. 100, 5280-5285 (2003).

27. Ewing, A. D. \& Kazazian, H. H., Jr. High-throughput sequencing reveals extensive variation in human-specific L1 content in individual human genomes. Genome Res. 20, 1262-1270 (2010).

28. Richardson, S. R. et al. Heritable L1 retrotransposition in the mouse primordial germline and early embryo. Genome Res. 27, 1395-1405 (2017).

29. Stewart, C. et al. A comprehensive map of mobile element insertion polymorphisms in humans. PLoS Genet. 7, e1002236 (2011).

30. Feusier, J. et al. Pedigree-based estimation of human mobile element retrotransposition rates. Genome Res. 29, 1567-1577 (2019).

31. de la Rica, L. et al. TET-dependent regulation of retrotransposable elements in mouse 
embryonic stem cells. Genome Biol. 17, 234 (2016).

838

839

840

841

842

843

844

845

846

847

848

849

850

851

852

853

854

855

856

857

858

859

860

861

862

863

864

865

866

867

32. Warkocki, Z. et al. Uridylation by TUT4/7 Restricts Retrotransposition of Human LINE-1s. Cell 174, 1537-1548.e29 (2018).

33. Deniz, Ö., Frost, J. M. \& Branco, M. R. Regulation of transposable elements by DNA modifications. Nat. Rev. Genet. 20, 417-431 (2019).

34. Sanchez-Luque, F. J. et al. LINE-1 Evasion of Epigenetic Repression in Humans. Mol. Cell 75, 590-604 (2019).

35. Ewing, A. D. et al. Nanopore Sequencing Enables Comprehensive Transposable Element Epigenomic Profiling. Mol. Cell 80, 915-928.e5 (2020).

36. Bourc'his, D. \& Bestor, T. H. Meiotic catastrophe and retrotransposon reactivation in male germ cells lacking Dnmt3L. Nature 431, 96-99 (2004).

37. Walter, M., Teissandier, A., Pérez-Palacios, R. \& Bourc'his, D. An epigenetic switch ensures transposon repression upon dynamic loss of DNA methylation in embryonic stem cells. Elife 5, e11418 (2016).

38. Greenberg, M. V. C. \& Bourc'his, D. The diverse roles of DNA methylation in mammalian development and disease. Nat. Rev. Mol. Cell Biol. 20, 590-607 (2019).

39. Goodier, J. L. Restricting retrotransposons: a review. Mob. DNA 7, 16 (2016).

40. Chelmicki, T. et al. m6A RNA methylation regulates the fate of endogenous retroviruses. Nature 591, 312-316 (2021).

41. MacLennan, M. et al. Mobilization of LINE-1 retrotransposons is restricted by Tex19.1 in mouse embryonic stem cells. Elife 6, (2017).

42. Salvador-Palomeque, C. et al. Dynamic Methylation of an L1 Transduction Family during Reprogramming and Neurodifferentiation. Mol. Cell. Biol. 39, (2019).

43. Lee, D.-S. et al. An epigenomic roadmap to induced pluripotency reveals DNA methylation as a reprogramming modulator. Nat. Commun. 5, 5619 (2014).

44. Knaupp, A. S. et al. Transient and Permanent Reconfiguration of Chromatin and Transcription Factor Occupancy Drive Reprogramming. Cell Stem Cell 21, 834-845.e6 (2017).

45. Klawitter, S. et al. Reprogramming triggers endogenous L1 and Alu retrotransposition in human induced pluripotent stem cells. Nat. Commun. 7, e10286 (2016).

46. Friedli, M. et al. Loss of transcriptional control over endogenous retroelements during 
reprogramming to pluripotency. Genome Res. 24, 1251-1259 (2014).

47. Maherali, N. et al. Directly reprogrammed fibroblasts show global epigenetic remodeling and widespread tissue contribution. Cell Stem Cell 1, 55-70 (2007).

48. Habibi, E. et al. Whole-genome bisulfite sequencing of two distinct interconvertible DNA methylomes of mouse embryonic stem cells. Cell Stem Cell 13, 360-369 (2013).

49. Garcia-Perez, J. L. et al. LINE-1 retrotransposition in human embryonic stem cells. Hum. Mol. Genet. 16, 1569-1577 (2007).

50. Macia, A. et al. Engineered LINE-1 retrotransposition in nondividing human neurons. Genome Res. 27, 335-348 (2017).

51. Wissing, S. et al. Reprogramming somatic cells into iPS cells activates LINE-1 retroelement mobility. Hum. Mol. Genet. 21, 208-218 (2012).

52. Stadtfeld, M., Maherali, N., Borkent, M. \& Hochedlinger, K. A reprogrammable mouse strain from gene-targeted embryonic stem cells. Nat. Methods 7, 53-55 (2010).

53. McKenna, A. et al. The Genome Analysis Toolkit: a MapReduce framework for analyzing next-generation DNA sequencing data. Genome Res. 20, 1297-1303 (2010).

54. Garrison, E. \& Marth, G. Haplotype-based variant detection from short-read sequencing. arXiv [q-bio.GN] arXiv:1207.3907 (2012).

55. Keane, T. M. et al. Mouse genomic variation and its effect on phenotypes and gene regulation. Nature 477, 289-294 (2011).

56. Rausch, T. et al. DELLY: structural variant discovery by integrated paired-end and splitread analysis. Bioinformatics 28, i333-i339 (2012).

57. Cameron, D. L. et al. GRIDSS: sensitive and specific genomic rearrangement detection using positional de Bruijn graph assembly. Genome Res. 27, 2050-2060 (2017).

58. Ewing, A. D. Transposable element detection from whole genome sequence data. Mob. DNA 6, 24 (2015).

59. Carreira, P. E. et al. Evidence for L1-associated DNA rearrangements and negligible L1 retrotransposition in glioblastoma multiforme. Mob. DNA 7, 21 (2016).

60. Schauer, S. N. et al. L1 retrotransposition is a common feature of mammalian hepatocarcinogenesis. Genome Res. 28, 639-653 (2018).

61. Ostertag, E. M. \& Kazazian, H. H., Jr. Twin priming: a proposed mechanism for the creation of inversions in L1 retrotransposition. Genome Res. 11, 2059-2065 (2001). 
62. Kano, H. et al. L1 retrotransposition occurs mainly in embryogenesis and creates somatic mosaicism. Genes Dev. 23, 1303-1312 (2009).

63. van den Hurk, J. A. J. M. et al. L1 retrotransposition can occur early in human embryonic development. Hum. Mol. Genet. 16, 1587-1592 (2007).

64. Sultana, T. et al. The Landscape of L1 Retrotransposons in the Human Genome Is Shaped by Pre-insertion Sequence Biases and Post-insertion Selection. Mol. Cell 74, 555-570 (2019).

65. Flasch, D. A. et al. Genome-wide de novo L1 Retrotransposition Connects Endonuclease Activity with Replication. Cell 177, 837-851 (2019).

66. Kopera, H. C. et al. LINE-1 cultured cell retrotransposition assay. Methods Mol. Biol. 1400, 139-156 (2016).

67. Han, J. S. \& Boeke, J. D. A highly active synthetic mammalian retrotransposon. Nature 429, 314-318 (2004).

68. Meyer, T. J., Srikanta, D., Conlin, E. M. \& Batzer, M. A. Heads or tails: L1 insertionassociated 5' homopolymeric sequences. Mob. DNA 1, 7 (2010).

69. Miki, Y. et al. Disruption of the APC gene by a retrotransposal insertion of L1 sequence in a colon cancer. Cancer Res. 52, 643-645 (1992).

70. Goodier, J. L., Ostertag, E. M. \& Kazazian, H. H., Jr. Transduction of 3'-flanking sequences is common in L1 retrotransposition. Hum. Mol. Genet. 9, 653-657 (2000).

71. Pickeral, O. K., Makałowski, W., Boguski, M. S. \& Boeke, J. D. Frequent human genomic DNA transduction driven by LINE-1 retrotransposition. Genome Res. 10, 411-415 (2000).

72. Moran, J. V., DeBerardinis, R. J. \& Kazazian, H. H., Jr. Exon shuffling by L1 retrotransposition. Science 283, 1530-1534 (1999).

73. Holmes, S. E., Dombroski, B. A., Krebs, C. M., Boehm, C. D. \& Kazazian, H. H., Jr. A new retrotransposable human L1 element from the LRE2 locus on chromosome 1q produces a chimaeric insertion. Nat. Genet. 7, 143-148 (1994).

74. Banuelos-Sanchez, G. et al. Synthesis and Characterization of Specific Reverse Transcriptase Inhibitors for Mammalian LINE-1 Retrotransposons. Cell Chem Biol 26, 1095-1109.e14 (2019).

75. Dai, L., Huang, Q. \& Boeke, J. D. Effect of reverse transcriptase inhibitors on LINE-1 and Ty1 reverse transcriptase activities and on LINE-1 retrotransposition. BMC Biochem. 12, 18 
(2011).

76. Smits, N. et al. No evidence of human genome integration of SARS-CoV-2 found by longread DNA sequencing. Cell Rep. 36, 109530 (2021).

77. Lanciano, S. \& Cristofari, G. Measuring and interpreting transposable element expression. Nat. Rev. Genet. 21, 721-736 (2020).

78. Zhou, W. et al. Identification and characterization of occult human-specific LINE-1 insertions using long-read sequencing technology. Nucleic Acids Res. 48, 1146-1163 (2020).

79. Wolf, G. et al. KRAB-zinc finger protein gene expansion in response to active retrotransposons in the murine lineage. Elife 9, e56337 (2020).

80. Heidmann, O. \& Heidmann, T. Retrotransposition of a mouse IAP sequence tagged with an indicator gene. Cell 64, 159-170 (1991).

81. Cheetham, S. W., Kindlova, M. \& Ewing, A. D. Methylartist: Tools for Visualising Modified Bases from Nanopore Sequence Data. bioRxiv (2021) doi:10.1101/2021.07.22.453313.

82. Scott, E. C. et al. A hot L1 retrotransposon evades somatic repression and initiates human colorectal cancer. Genome Res. 26, 745-755 (2016).

83. Shakiba, N. et al. Cell competition during reprogramming gives rise to dominant clones. Science 364, (2019).

84. Saini, N. et al. The Impact of Environmental and Endogenous Damage on Somatic Mutation Load in Human Skin Fibroblasts. PLoS Genet. 12, e1006385 (2016).

85. Macia, A. et al. Epigenetic control of retrotransposon expression in human embryonic stem cells. Mol. Cell. Biol. 31, 300-316 (2011).

86. Grandi, F. C. et al. Retrotransposition creates sloping shores: a graded influence of hypomethylated CpG islands on flanking CpG sites. Genome Res. 25, 1135-1146 (2015).

87. Dewannieux, M. \& Heidmann, T. L1-mediated retrotransposition of murine B1 and B2 SINEs recapitulated in cultured cells. J. Mol. Biol. 349, 241-247 (2005).

88. Dewannieux, M., Esnault, C. \& Heidmann, T. LINE-mediated retrotransposition of marked Alu sequences. Nat. Genet. 35, 41-48 (2003).

89. Firas, J., Liu, X., Nefzger, C. M. \& Polo, J. M. GM-CSF and MEF-conditioned media support feeder-free reprogramming of mouse granulocytes to iPS cells. Differentiation 87, 
193-199 (2014).

90. Bertoncello, I. \& McQualter, J. Isolation and clonal assay of adult lung epithelial stem/progenitor cells. Current Protocols in Stem Cell Biology 16, (2011).

91. Seach, N., Wong, K., Hammett, M., Boyd, R. L. \& Chidgey, A. P. Purified enzymes improve isolation and characterization of the adult thymic epithelium. J. Immunol. Methods 385, 23-34 (2012).

92. Merlos-Suárez, A. et al. The intestinal stem cell signature identifies colorectal cancer stem cells and predicts disease relapse. Cell Stem Cell 8, 511-524 (2011).

93. Jensen, K. B., Driskell, R. R. \& Watt, F. M. Assaying proliferation and differentiation capacity of stem cells using disaggregated adult mouse epidermis. Nat. Protoc. 5, 898-911 (2010).

94. Alaei, S. et al. An improved reprogrammable mouse model harbouring the reverse tetracycline-controlled transcriptional transactivator 3. Stem Cell Res. 17, 49-53 (2016).

95. Lim, S. M. et al. Temporal restriction of pancreatic branching competence during embryogenesis is mirrored in differentiating embryonic stem cells. Stem Cells Dev. 21, 1662-1674 (2012).

96. Liu, K. et al. KSR-based medium improves the generation of high-quality mouse iPS cells. PLoS One 9, e105309 (2014).

97. Knaupp, A. S. et al. TINC-A Method to Dissect Regulatory Complexes at Single-Locus Resolution-Reveals an Extensive Protein Complex at the Nanog Promoter. Stem cell reports 15, 1246-1259 (2020).

98. Li, H. Aligning sequence reads, clone sequences and assembly contigs with BWA-MEM. arXiv [q-bio.GN] arXiv:1303.3997 (2013).

99. Cingolani, P. et al. A program for annotating and predicting the effects of single nucleotide polymorphisms, SnpEff: SNPs in the genome of Drosophila melanogaster strain w1118; iso-2; iso-3. Fly 6, 80-92 (2012).

100. Kent, W. J. et al. The human genome browser at UCSC. Genome Res. 12, 996-1006 (2002).

101. Bao, W., Kojima, K. K. \& Kohany, O. Repbase Update, a database of repetitive elements in eukaryotic genomes. Mob. DNA 6, 11 (2015).

102. Untergasser, A. et al. Primer3--new capabilities and interfaces. Nucleic Acids Res. 40, e115 (2012). 
103. Martin, S. L. et al. A single amino acid substitution in ORF1 dramatically decreases L1 retrotransposition and provides insight into nucleic acid chaperone activity. Nucleic Acids Res. 36, 5845-5854 (2008).

104. Ostertag, E. M., Prak, E. T., DeBerardinis, R. J., Moran, J. V. \& Kazazian, H. H., Jr. Determination of L1 retrotransposition kinetics in cultured cells. Nucleic Acids Res. 28, 1418-1423 (2000).

105. Freeman, J. D., Goodchild, N. L. \& Mager, D. L. A modified indicator gene for selection of retrotransposition events in mammalian cells. Biotechniques 17, 46, 48-9, 52 (1994).

106. Kumaki, Y., Oda, M. \& Okano, M. QUMA: quantification tool for methylation analysis. Nucleic Acids Res. 36, W170-5 (2008).

107. Li, H. Minimap2: pairwise alignment for nucleotide sequences. Bioinformatics 34, 30943100 (2018).

108. Li, H. et al. The Sequence Alignment/Map format and SAMtools. Bioinformatics 25, 20782079 (2009).

109. Simpson, J. T. et al. Detecting DNA cytosine methylation using nanopore sequencing. Nat. Methods 14, 407-410 (2017).

110. Dreos, R., Ambrosini, G., Groux, R., Cavin Périer, R. \& Bucher, P. The eukaryotic promoter database in its 30th year: focus on non-vertebrate organisms. Nucleic Acids Res. 45, D51-D55 (2017).

\section{Acknowledgements}

The authors thank Jef D. Boeke and John V. Moran for sharing L1SM plasmids and the HeLaJVM cell line, respectively. The study was supported by an Australian Government Research Training Program (RTP) Scholarship and a Mater Research Frank Clair Scholarship awarded to P.G., ARC Discovery Early Career Researcher Award (DE150101117), Discovery Project (DP170101198) and Australian Department of Health Medical Frontiers Future Fund (MRFF) (MRF1175457) grants awarded to A.D.E., NHMRC Project Grant (GNT1051117), ARC Future Fellowship (FT180100674) and Sylvia and Charles Viertel Senior Medical Research Fellowship funds awarded to J.M.P., NHMRC Investigator Grants (GNT1178460 to R.L., GNT1173476 to S.R.R., GNT1173711 to G.J.F.), and CSL Centenary Fellowship and NHMRC Project Grant (GNT1106206, GNT1125645, GNT1126393, GNT1138795) funding awarded to G.J.F.. A.D.E., S.R.R. and G.J.F. additionally acknowledge support from the Mater Foundation. 


\section{Author contributions}

1025 P.G., S.M.L., M.R.L., D.C., F.J.S-L., L.W., C.J., A.S.K., P.E.C., C.M.N. and S.R.R. performed 1026 experiments. R.L., J.M.P. and G.J.F. provided resources. A.D.E. and G.J.F. performed 1027 bioinformatic analyses. P.G., S.R.R., J.M.P. and G.J.F. conceived the study and designed 1028 experiments. P.G., S.R.R. and G.J.F. generated figures. G.J.F. wrote the manuscript and directed 1029 the study. All authors commented on the manuscript.

1030

1031 Competing interests

1032 The authors declare no competing interests. 

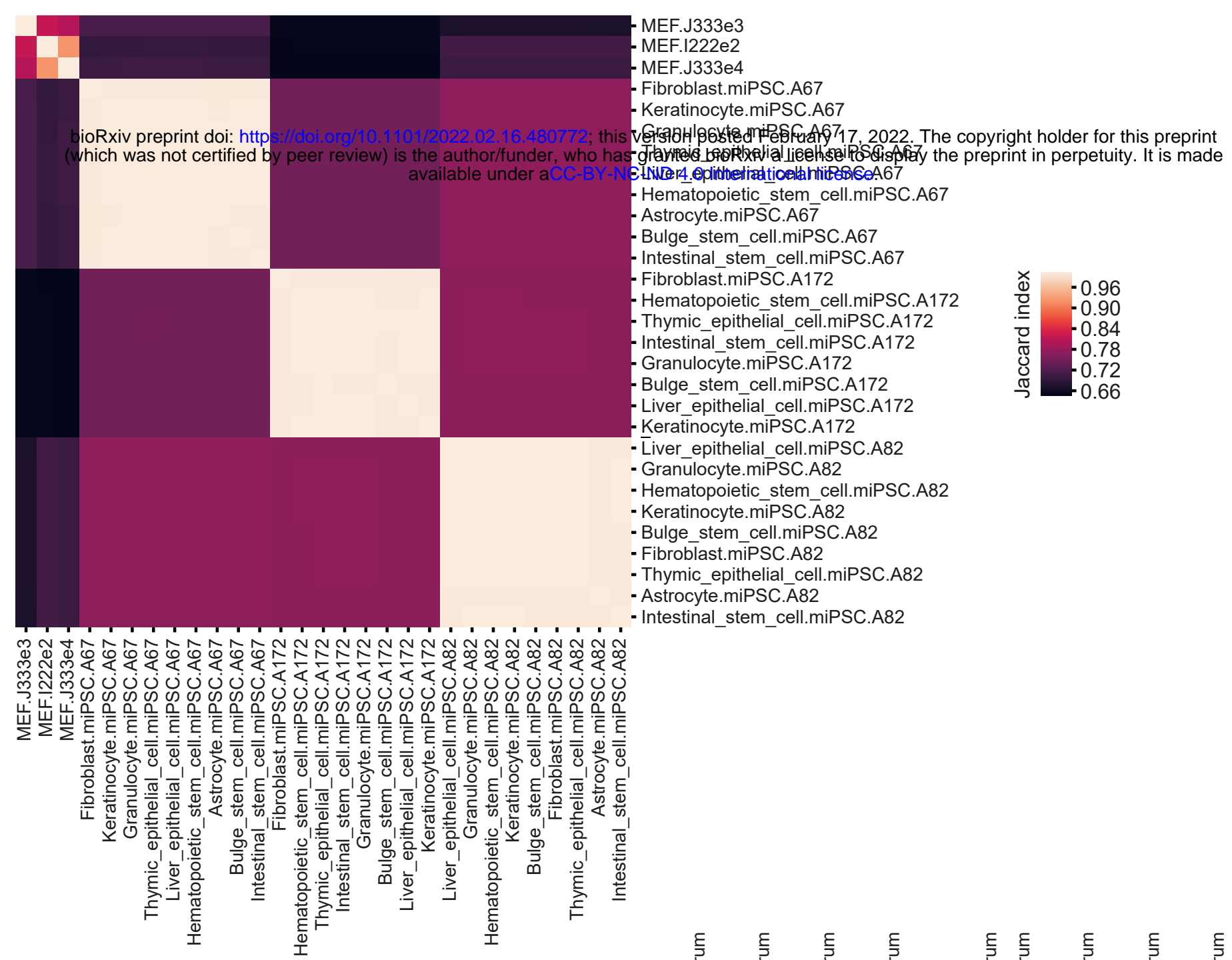

b
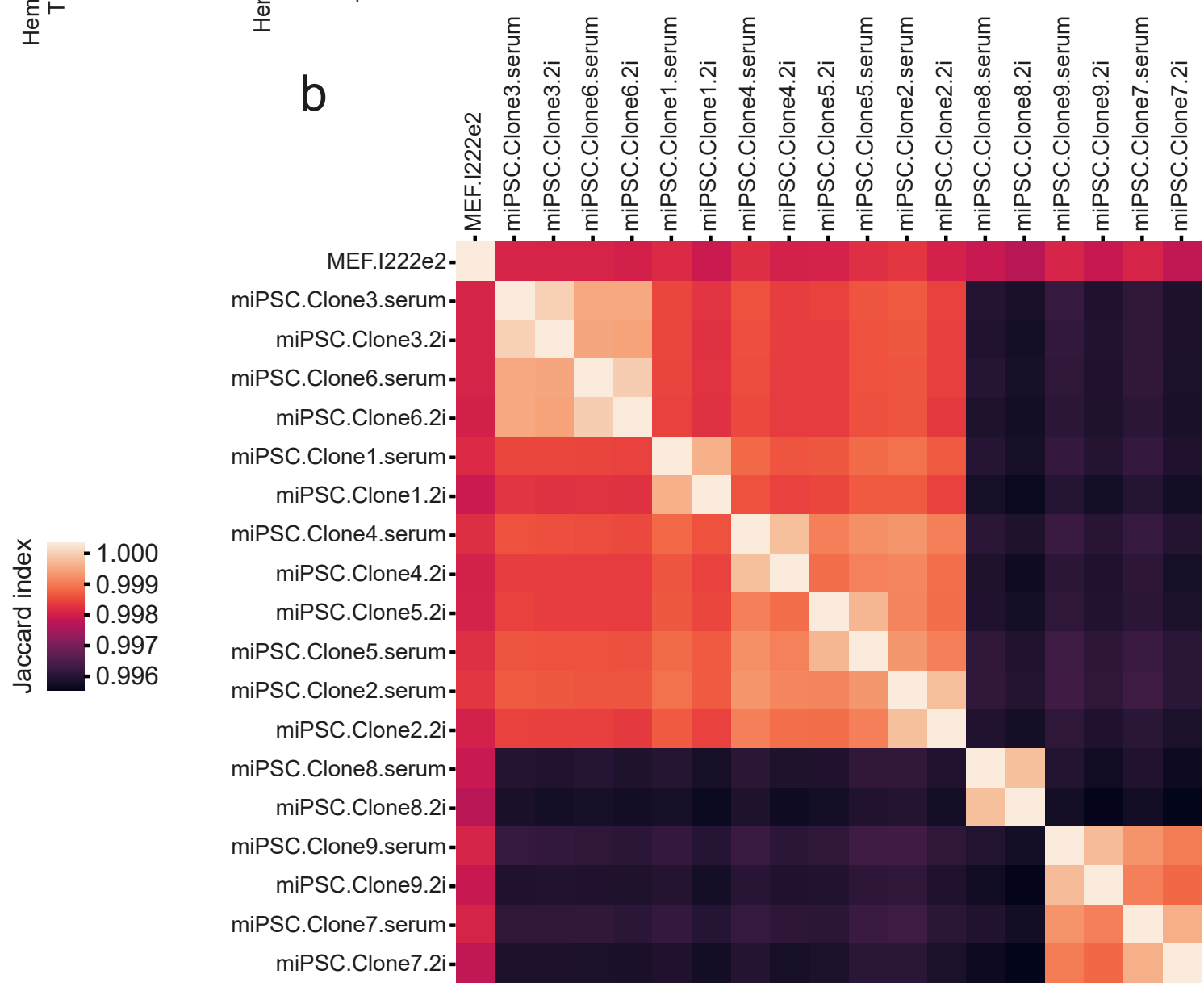

Extended Data Fig. 1: miPSC line genotypic relationships. a, Clustering of miPSC lines derived from 9 primary cell types isolated from 3 animals (A67, A82, A172), and 3 MEF genotypic controls. For each pairwise comparison, the Jaccard index (J) was calculated as the ratio of the union and intersection of SNP/INDEL variants called from WGS data and shared by the sample pair. Known SNPs/INDELs were removed and filtered as described in the Methods. $\mathrm{J}=1$ (light color on key) indicates an identical variant profile between a sample pair, whereas $\mathrm{J}=0$ (dark color on key) indicates no variants in common. Hierarchical clustering was performed using average linkage and a Euclidean distance metric via the seaborn clustermap function. b, As for panel (a), except for 9 single-cell clones derived from animal $\mathrm{I} 222 \mathrm{e} 2 \mathrm{MEFs}$ and cultured in serum or $2 \mathrm{i}$ conditions. 
Chr1 (qC5)

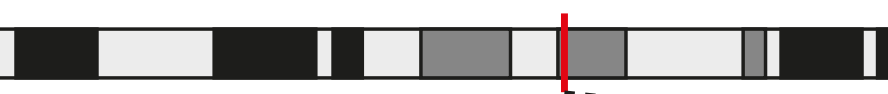

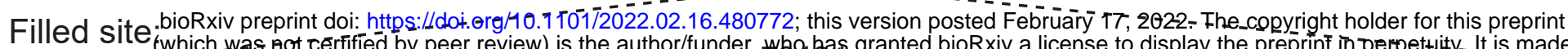
Filled site (whicb was not certified by peer review) is the author/funder, who bas granted bioRxiv a license to display the prepriñt in perpettiity- Lt is made

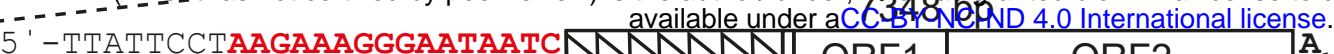

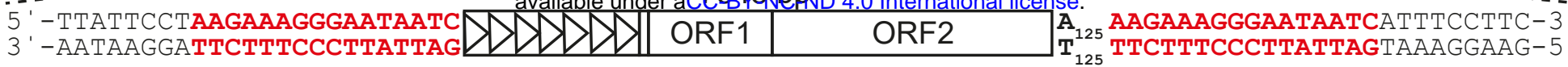

Empty site:

5 ' -TCTGACTCATCTCTGATTGAATTTACTTTTATTCCTAAGAAAGGGAATAATCATTTCCTTCACTTATTCTCAAGCTTAGCTTCTAAT-3

3 ' -AgACTGAGTAGAGACTAACTTAAATGAAAATAAGGATTCTTTCCCTTATtAGTAAAGGAAGTGAATAAGAGTTCGAATCGAAGATTA-5

\section{$\uparrow 1$ st strand cleavage}
L1 subfamily: $T_{F}$
Monomers: $6+182 \mathrm{bp}$
TSD: 16 bp
EN motif: 5'-TCTT/AG-3

miPSC_2_L1 (Chr10: 54,739,001-54,739,014; +strand)

Chr10 (qB3)

Filled site:

$3^{\prime}$ ' - A AGAAAGAAGCATGAAATATTCTATGAGGCACATAAGGCT-3
$\mathrm{T}_{>100}$ TCTTTCTTCGTACTTTATAAGATACTCCGTGTATTCCGA-5

Empty site:

5 ' -ACAACATAAAGAGAATATTCTGATCCTATAATGTGAACAGAAAGAAGCATGAAATATTCTATGAGGCACATAAGgCTTGAAACTGGT-3

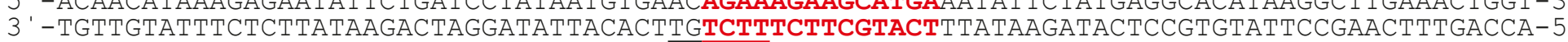
$\uparrow 1$ st strand cleavage
L1 subfamily: $T_{F}$
Monomers: 0
TSD: $14 \mathrm{bp}$
EN motif: 5'-TTCT/GT-3'

miPSC_3_L1 (Chr13: 35,290,163-35,290,177; +strand)

Chr13 (qA3.3

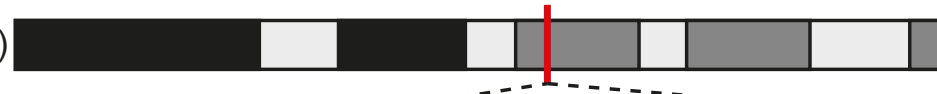

Filled site:

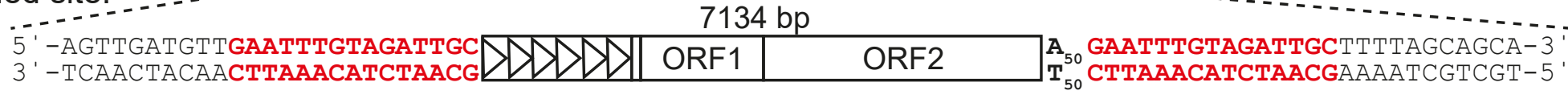

Empty site:

5 '-GTTGGACAGTTGGGGCTGCATGATGCAAGTTGATGTTGAATTTGTAGATTGCTTTAGCAGCACAGCCATTTTCATGATGTTAATTT-3

3 ' -CAACCTGTCAACCCCGACGTACTACGTTCAACTACAACTTAAACATCTAACGAAAATCGTCGTGTCGGTAAAAGTACTACAATTAAA-5

$\uparrow 1$ st strand cleavage
L1 subfamily: $T_{F}$
Monomers: $5+182$ bp
TSD: $15 \mathrm{bp}$
EN motif: 5 '-ATTC/AA-3

\section{miPSC_4_L1 (Chr3: 70,528,174-70,528,186; +strand)}

Chr3 (qE2)

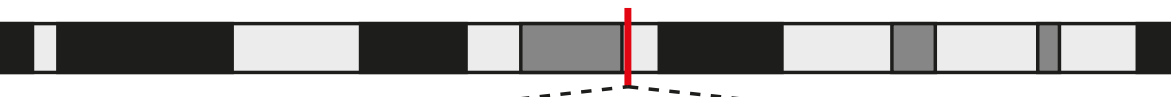

Filled site:
5 -ATAGTTGAGGATTAAGAACCCAATCA
3 ' - TATCAACTCCTAATTCTTGGGTTAGTD
$7023 \mathrm{bp}$

Empty site:

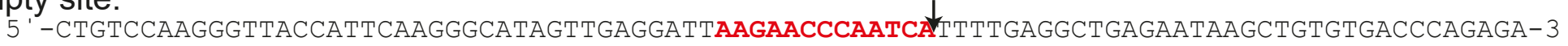

3 ' - GACAGGTTCCCAATGGTAAGTTCCCGTATCAACTCCTAATTCTTGGGTTAGTAAAACTCCGACTCTTATTCGACACACTGGGTCTCT-5 '
L1 subfamily: $T_{F}$
Monomers: $5+70$ bp
$\uparrow 1$ st strand cleavage

Extended Data Fig. 2: Sequence characteristics of de novo L1 insertions detected in bulk tissue-derived miPSCs. For each of four insertions, the following information is provided: the chromosomal location; a filled site illustration indicating target site duplication (TSD) sequences in red, the number of promoter monomers (black triangles) if applicable, and 3' polyA tract length $\left(\mathrm{A}_{\mathrm{n}} / \mathrm{T}_{\mathrm{n}}\right)$; an empty site illustration depicting TSD sequence and first strand endonuclease (EN) cleavage motif (underlined); summary characteristics (L1 subfamily, number of monomers, TSD length and EN motif). 


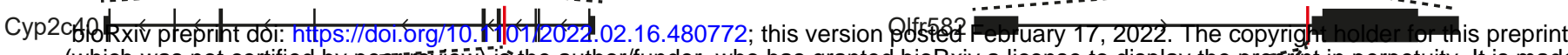
(which was not cectified by- peetreviêw) is the author/funder, who has granted bioRxiv a license to display the-preptint in perpetuity. It is made

miPSC_5_L1 1 GF

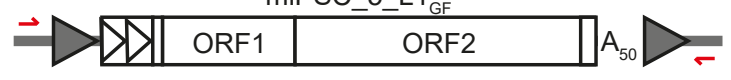

Animal A82 miPSC lines

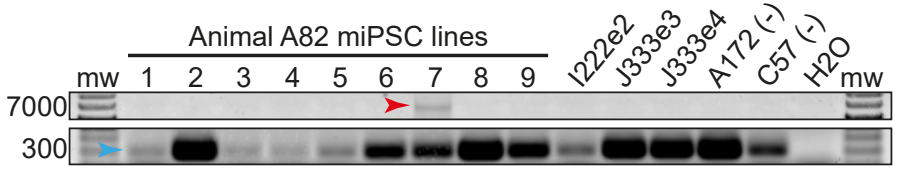

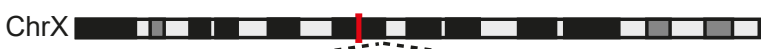
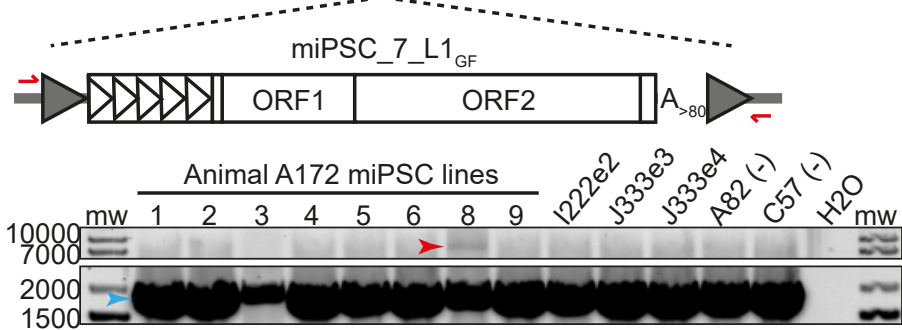

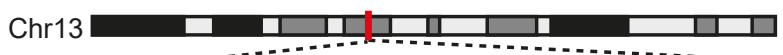

Fam120a

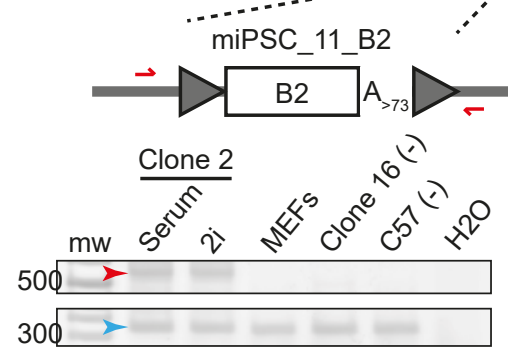

Chr15

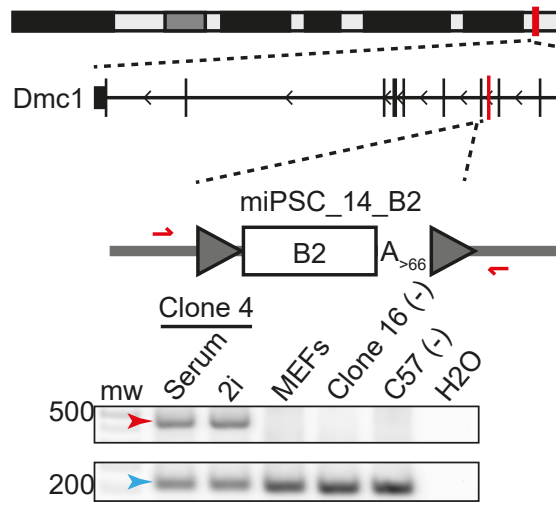

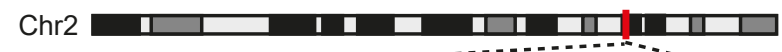

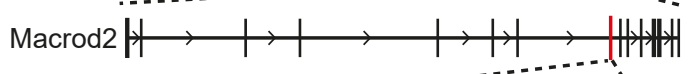

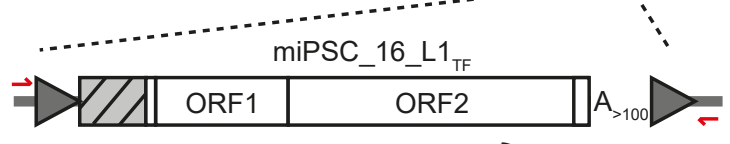

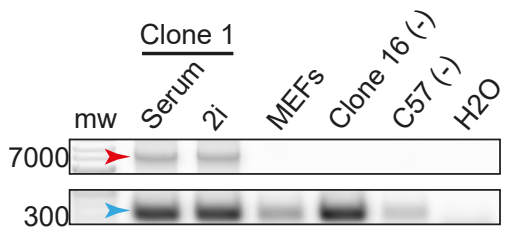

miPSC_6_L1 $1_{\text {TF }}$
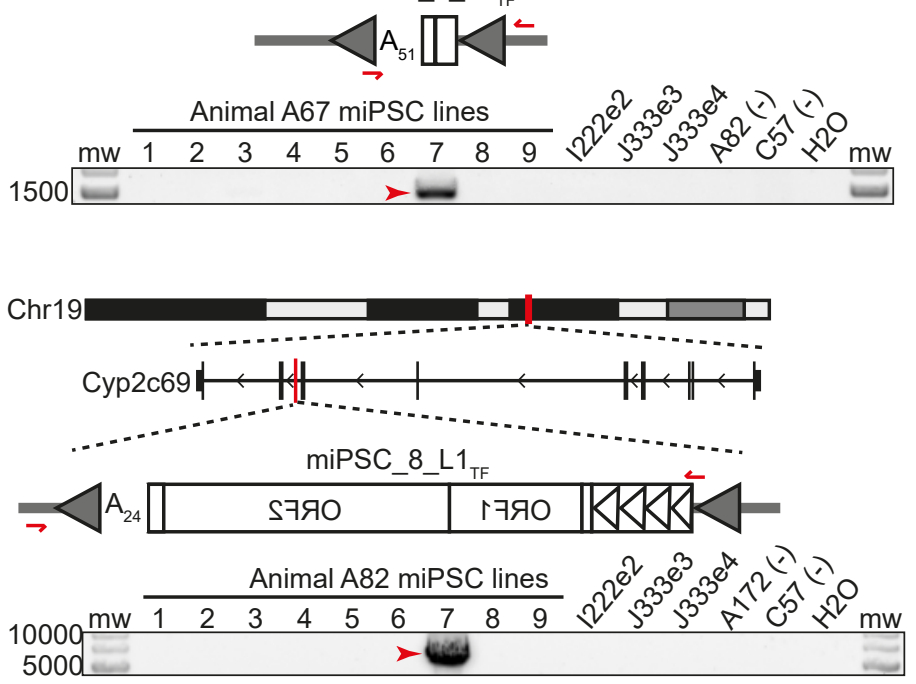

Chr13
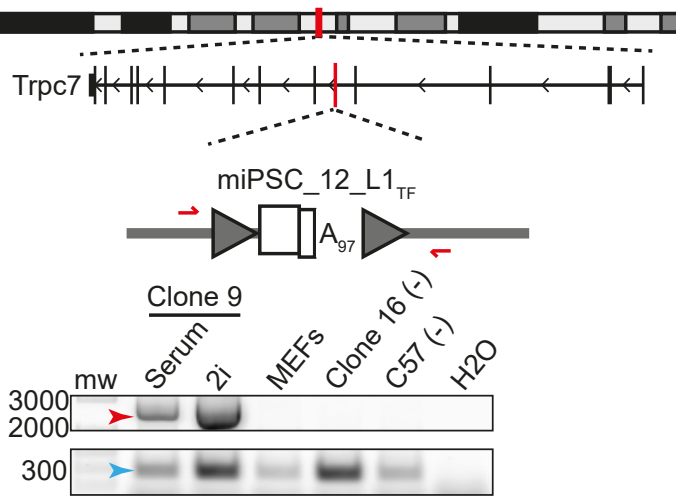

Chr2
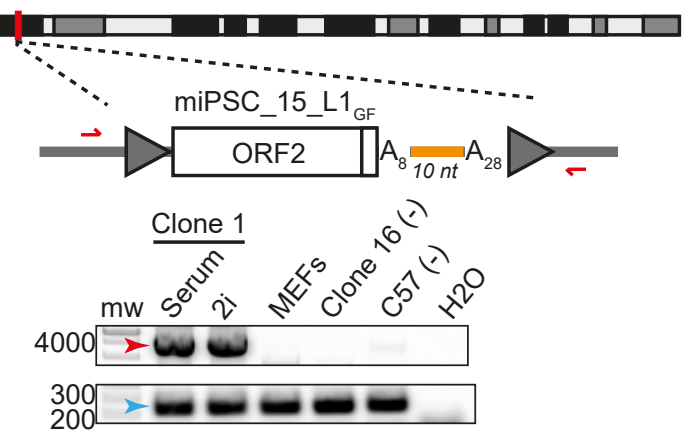

Chr3
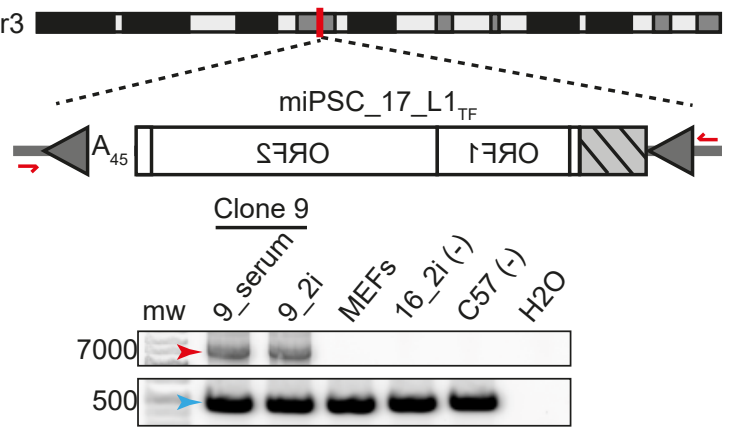


\section{$\because$}

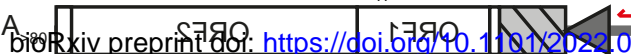
(which was not certified by peer,review) is the author/funder, who has granted bioRxiv a license tadispiely the preprint in perpetuity. It is made

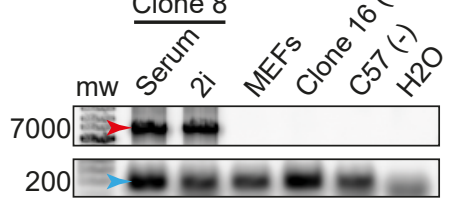
available under aCC-BY-NC-ND 4.0 International.jicense.

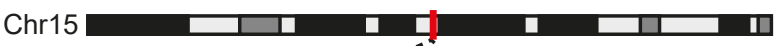
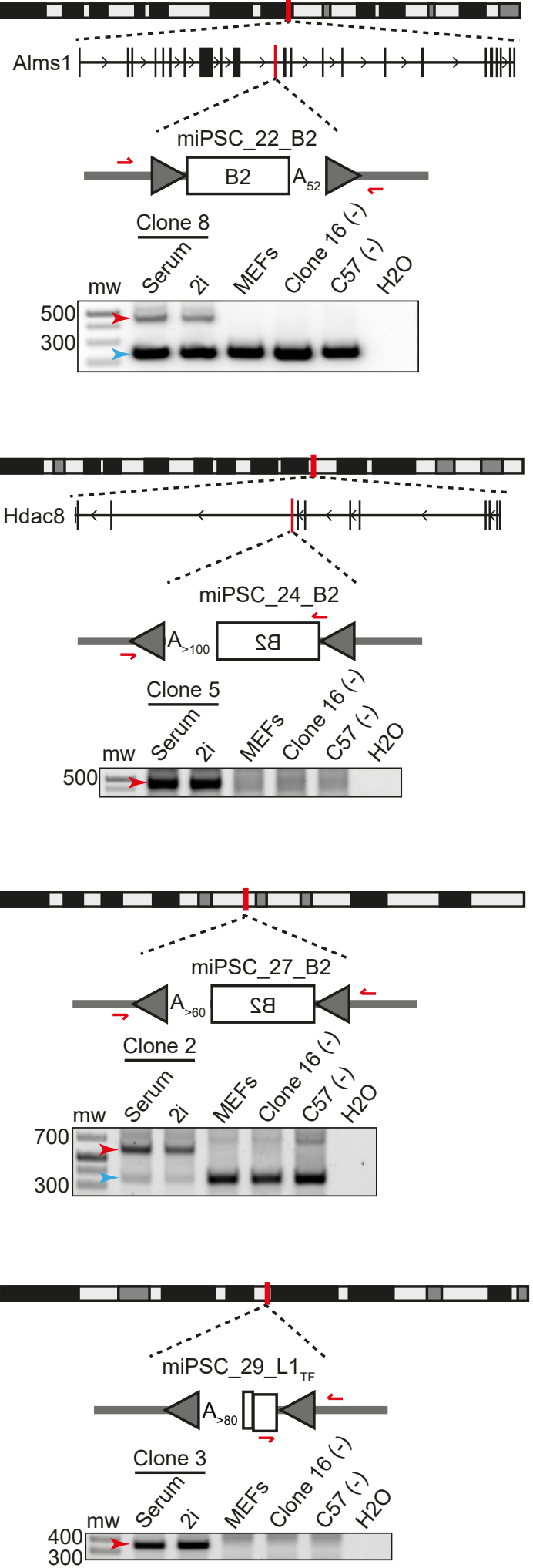

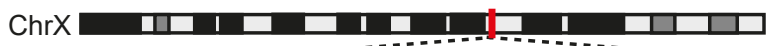
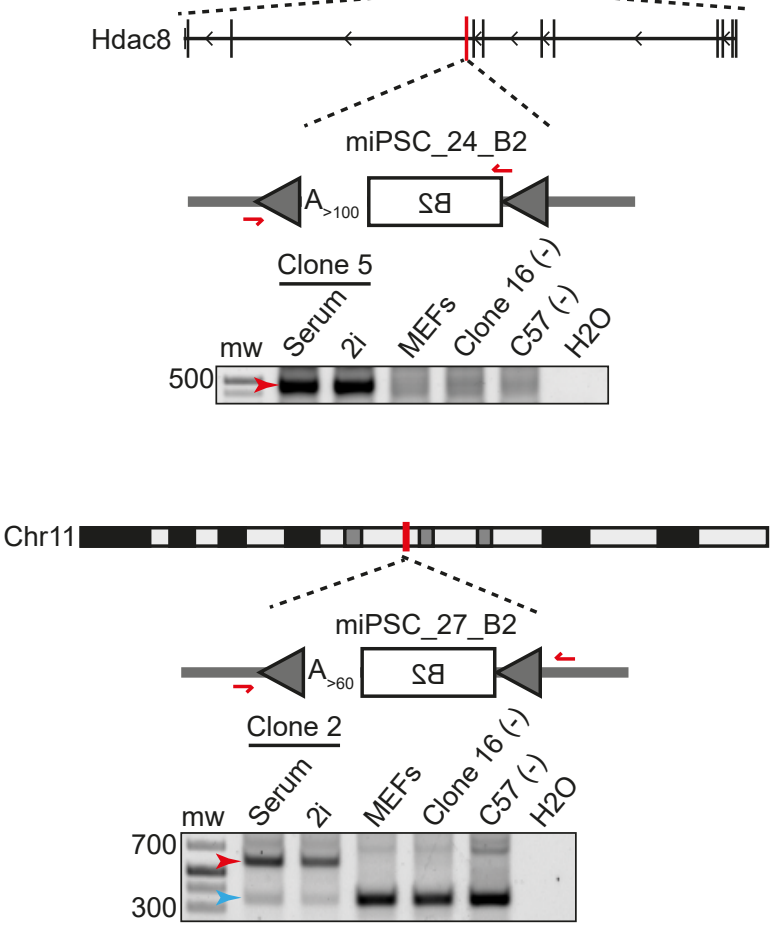

300

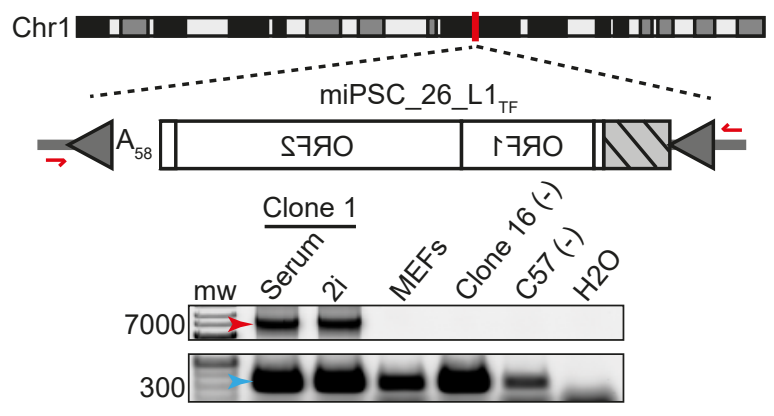

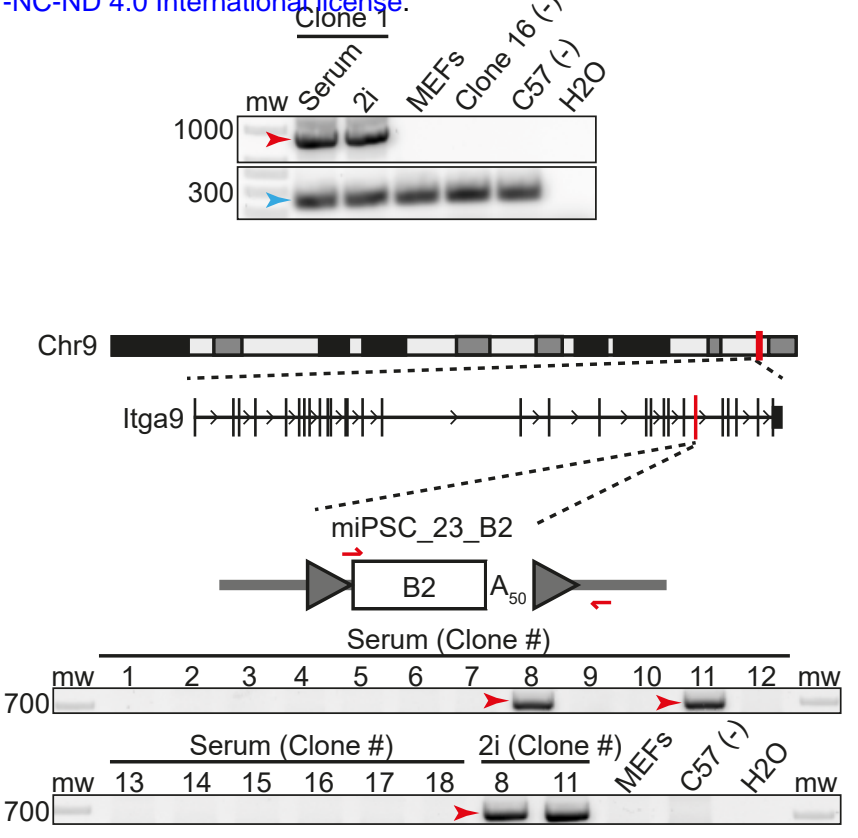
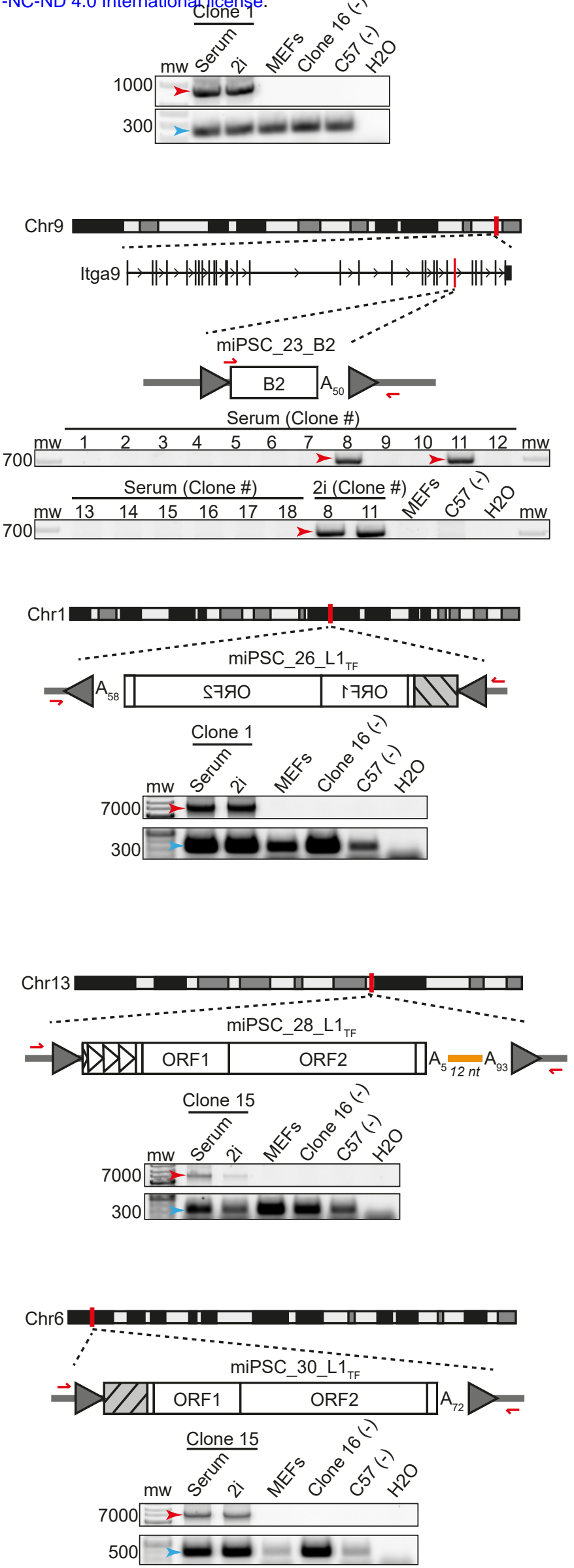


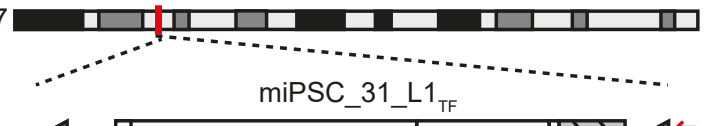

; this version posted February 7, 2022. The copyright thodder for this preptint (which was not certified dypeer deview) is the exthor/funder, who has granted bioRxiv a license to displaycthe preprint in perpeturity. It is made

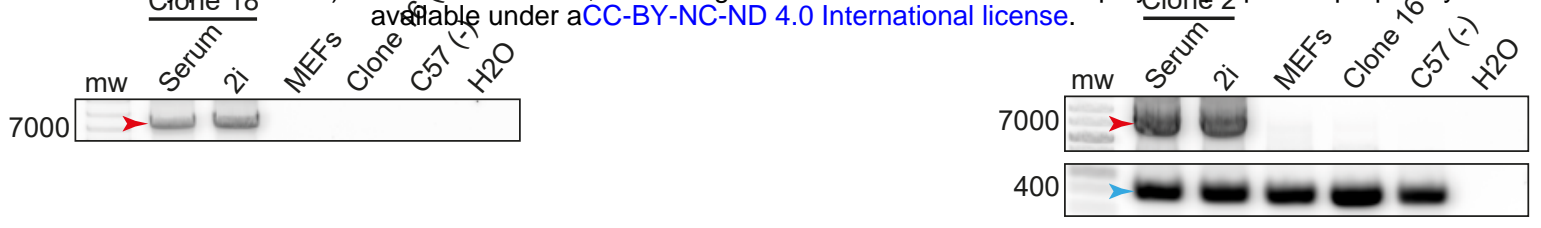

Chr8

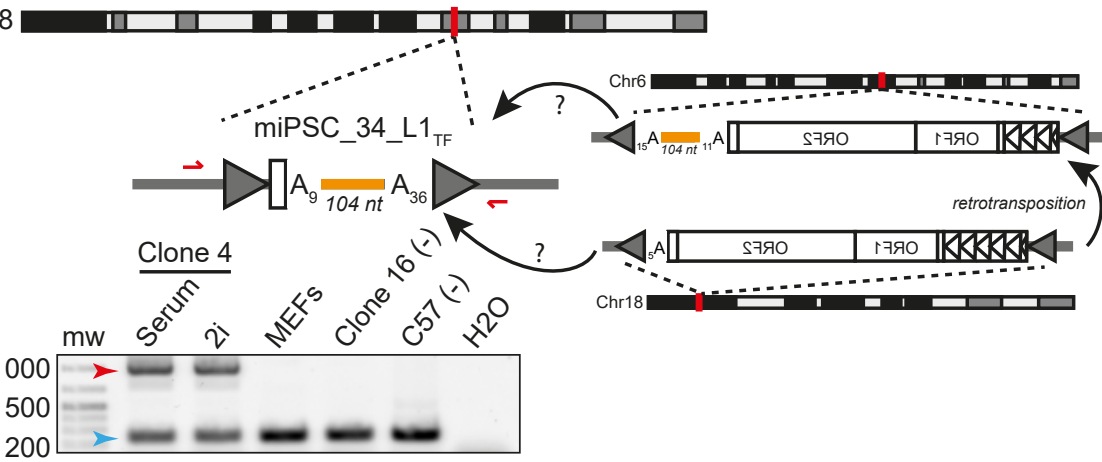

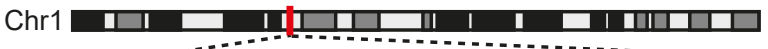
Pard3b $\mid$ | miPSC_35_L $1_{\mathrm{TF}}$ $\rightarrow \prod^{A_{38}}{ }_{\sigma}$

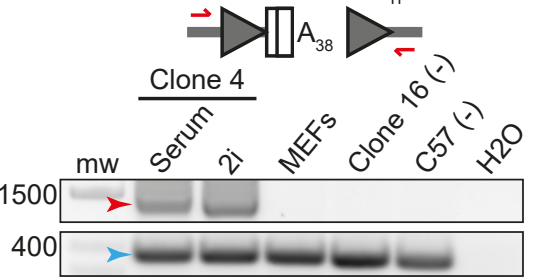

Chr1

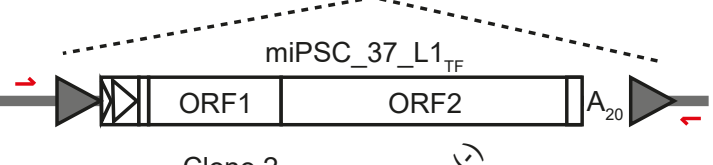

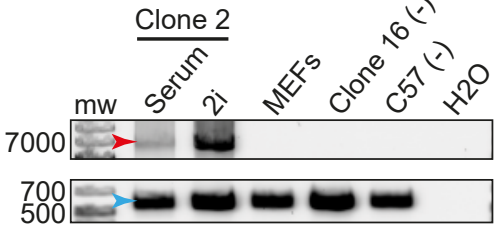

Chr10

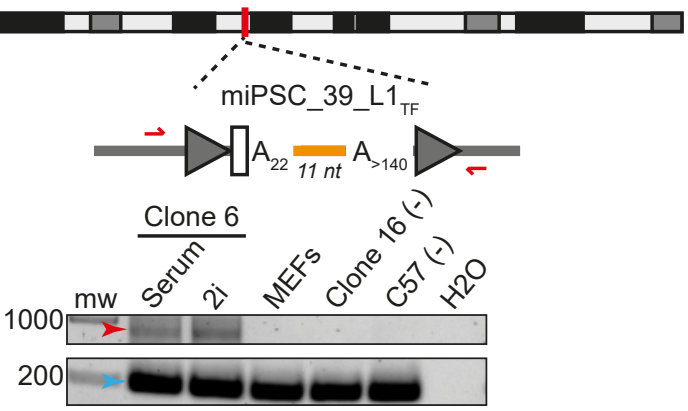

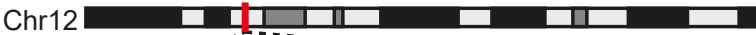

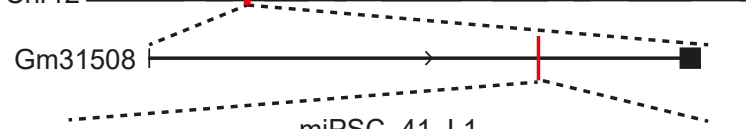

miPSC 41 L $1_{\text {TF }}$

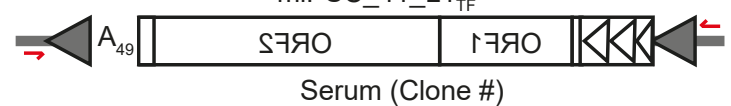

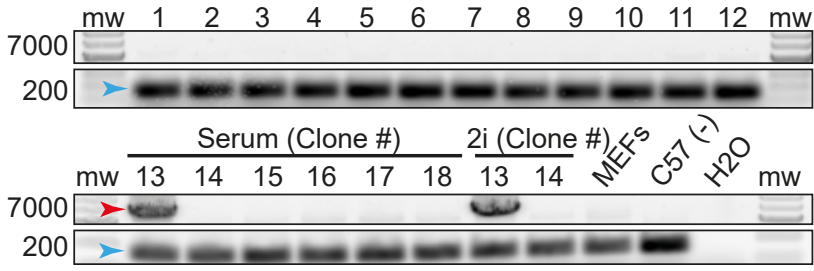




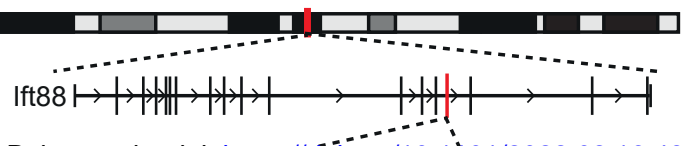

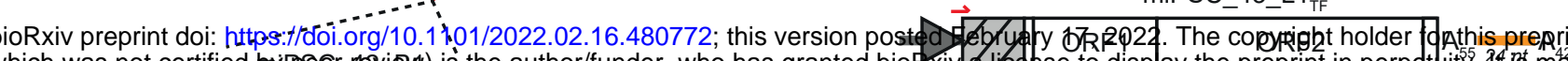
(which was not certified byipeer review) is the author/funder, who has granted bio

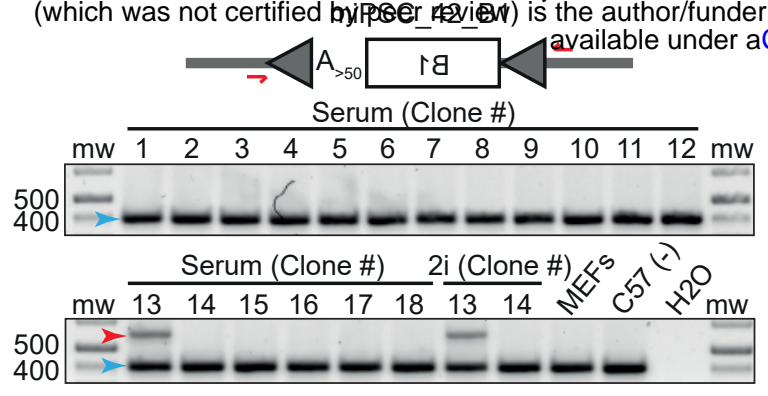
$\mathrm{mw} \begin{array}{llllllllllllll}1 & 2 & 3 & 4 & 5 & 6 & 7 & 8 & 9 & 10 & 11 & 12 & 13 & \mathrm{mw}\end{array}$

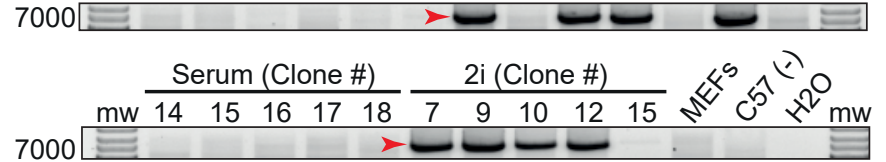

Extended Data Fig. 3: Additional de novo TE insertion validation and characterization. De novo TE insertions found in 26 bulk miPSC lines generated from primary cells or 18 single-cell miPSC clones derived from MEFs. For each insertion, the chromosomal location and orientation are shown. L1 and SINE B1 and B2 insertions are represented by white rectangles. L1 5'UTR promoter monomers, if present, are indicated by triangles or, if the number of monomers is unknown, a grey box with black stripes. Poly(A) tracts and their length are indicated $\left(\mathrm{A}_{\mathrm{n}}\right)$, and target site duplications (TSDs) are depicted as grey arrows. $3^{\prime}$ transductions are shown as orange lines. PCR validation primers are shown as red arrows. PCR products in agarose gels used to confirm TE insertions are indicated by red arrows. Empty site (wild-type) amplicons are indicated by blue arrows, where applicable. 
bigR*iv preprint doi: https://doi.org/10.1101/2022.02.16.480772; this version postedFebruary 17, 2022. The copyrigheholder for this preprint

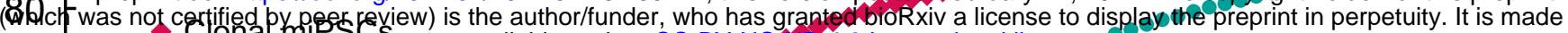

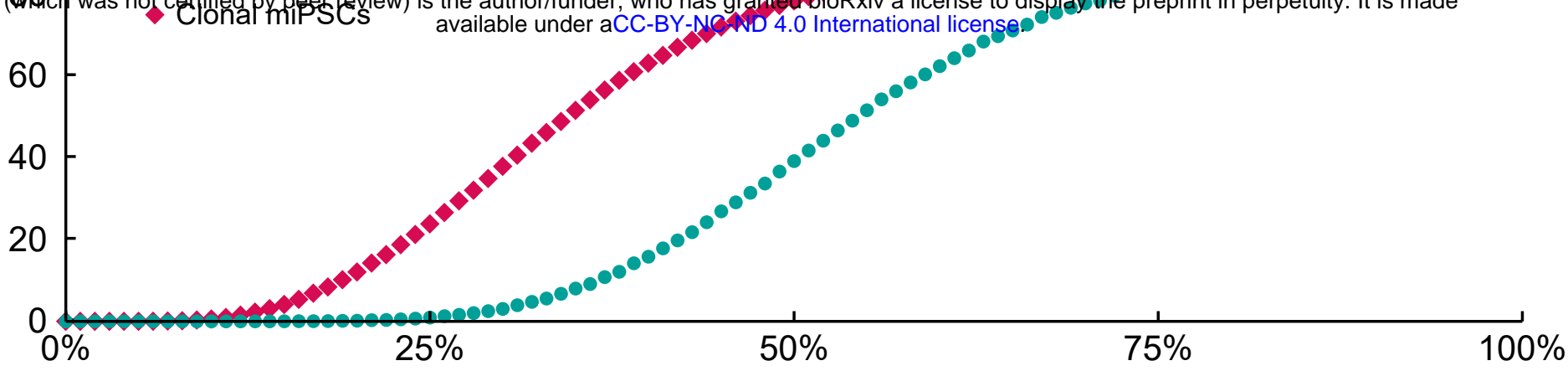

Relative WGS down sampling depth

b

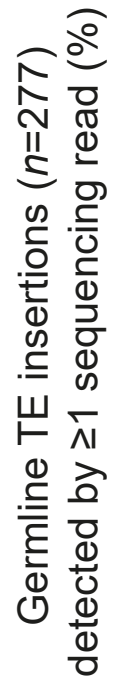

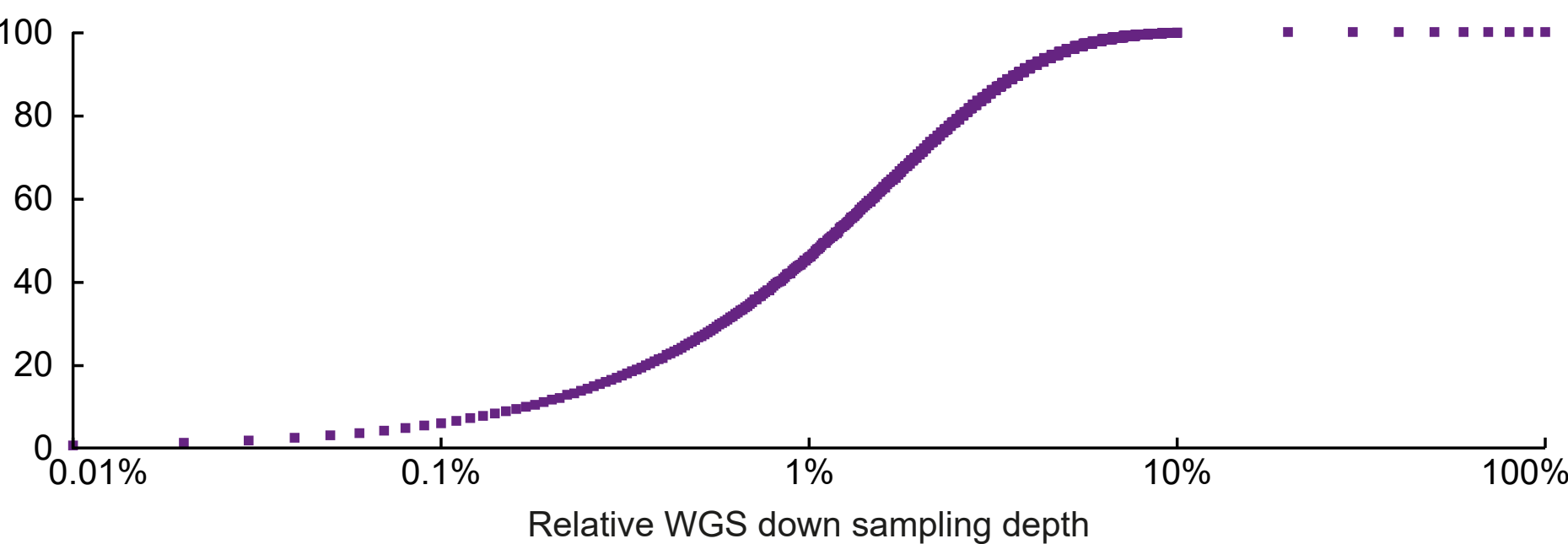

Extended Data Fig. 4: TE detection sensitivities at simulated sequencing depths. a, To assess whether PCR validated de novo TE insertions would have been initially overlooked by lower coverage WGS, we down sampled our $\sim 41 \times$ average depth WGS in percentile increments. In order to be called as present, de novo insertions found in the bulk (top) and single-cell (bottom) miPSC experiments required $\geq 1$ WGS read at each of their $5^{\prime}$ and $3^{\prime}$ junctions, and $\geq 10 \mathrm{WGS}$ reads in total. $\mathbf{b}$, To estimate the likelihood of a mosaic TE insertion being overlooked in the parental animal I222e2 MEF population, and called as de novo in one of the associated clonal miPSC lines, we defined a set of 277 heterozygous germline TE insertions found in I222e 2 and that were detected by $\geq 25$ WGS or mRC-seq reads at each of their $5^{\prime}$ and $3^{\prime}$ junctions. We then simulated the probability of at least one read being found for an insertion when the reads assigned to that insertion were assigned probabilities to achieve random sampling depths ranging from $0.01 \%$ to $100 \%$ of the parental MEF bulk sequencing data. Note: at each depth in panels (a) and (b), simulations were repeated 10,000 times. 


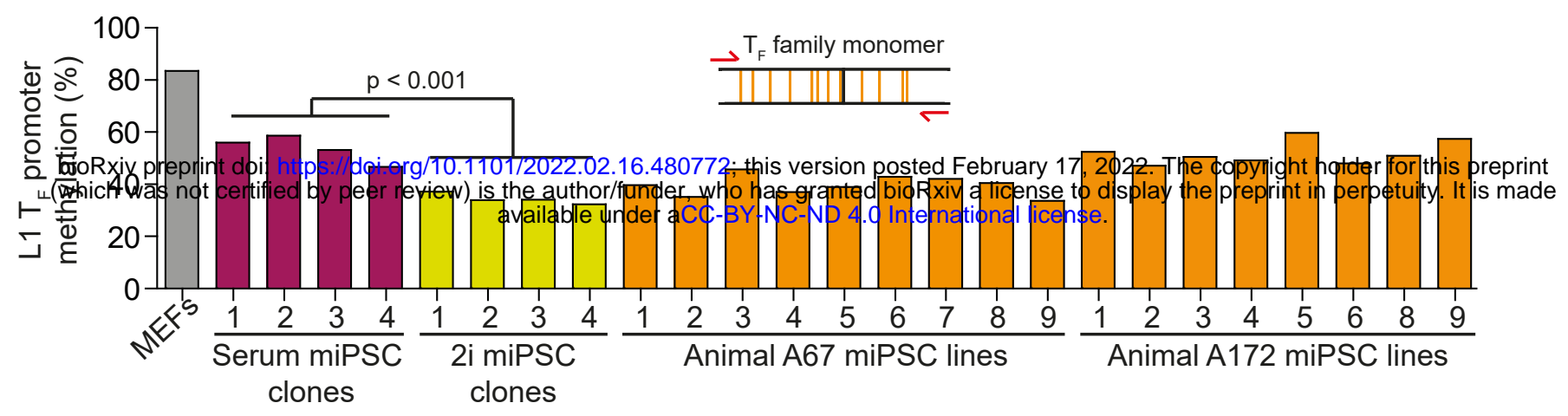

b

MEFs

(animal I222e2)

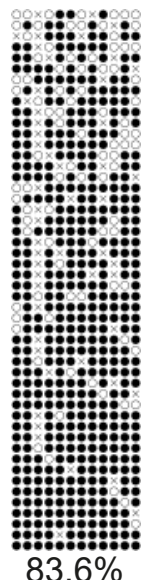

Serum miPSC clones

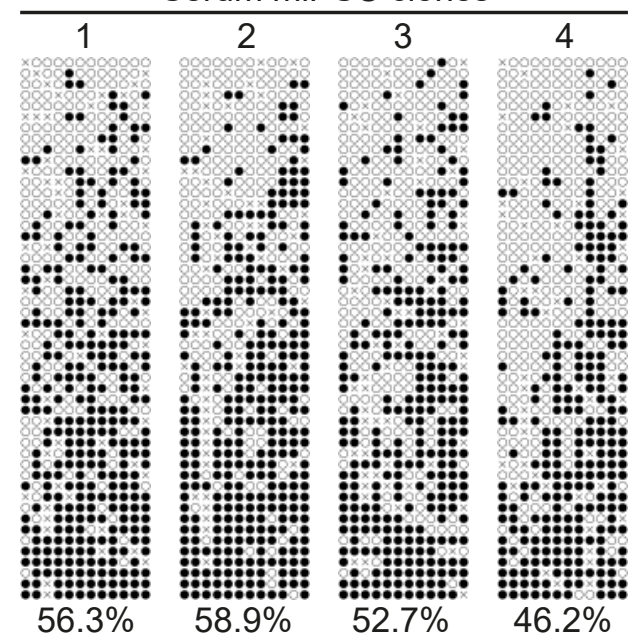

2i miPSC clones

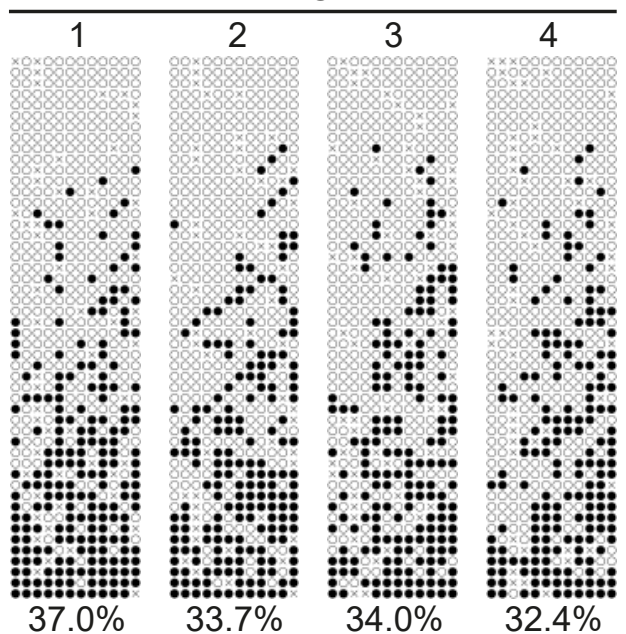

Animal A67 miPSC lines

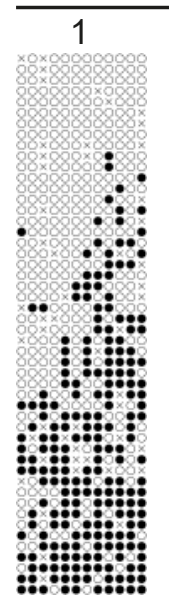

$39.7 \%$

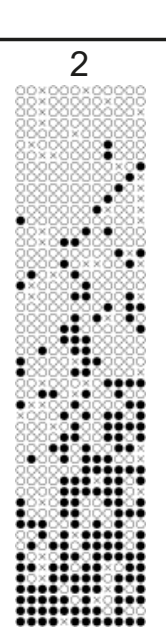

$35.5 \%$

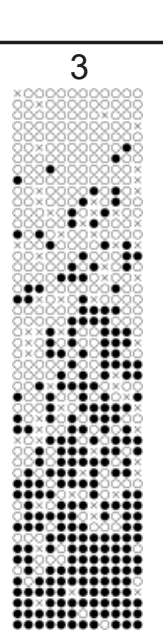

$4 \quad 5$

$45.9 \%$
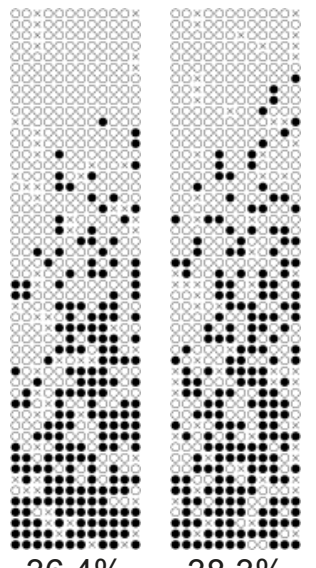

$36.4 \%$

$38.3 \%$

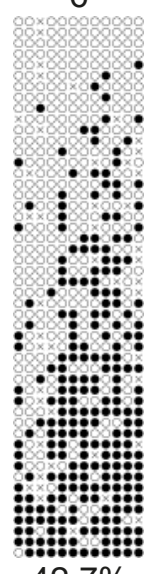

$42.7 \%$
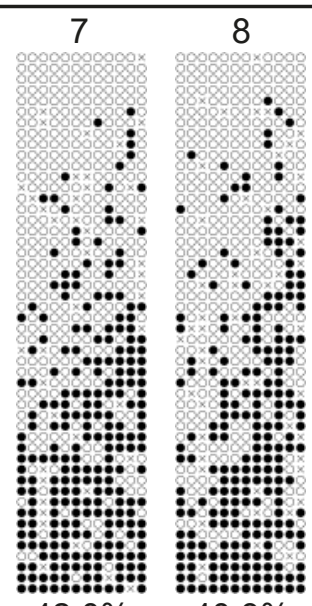

$42.0 \%$

$40.3 \%$

Animal A172 miPSC lines

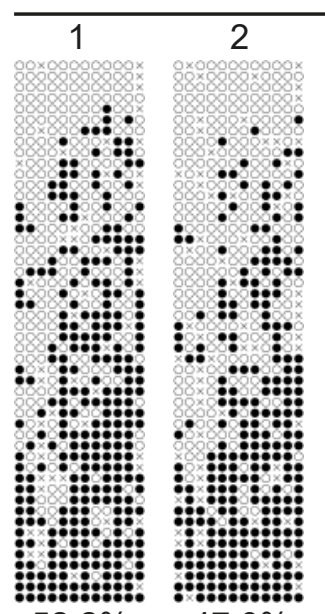

$52.2 \%$

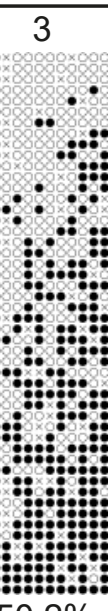

$50.2 \%$

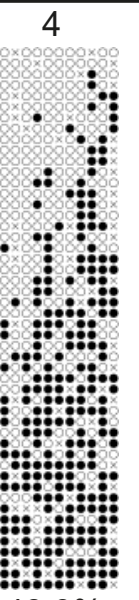

$48.9 \%$

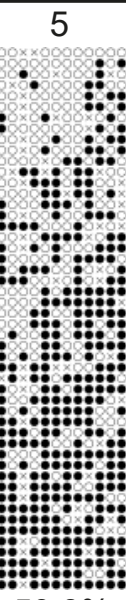

$59.8 \%$

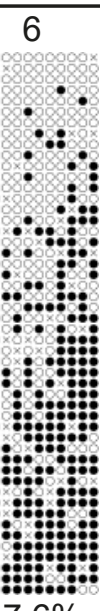

89

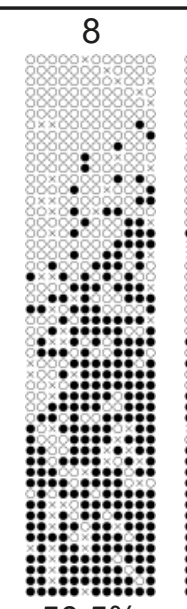

$50.5 \%$

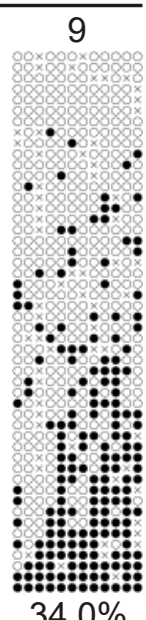

1: granulocytes

2: hematopoietic stem cells

3: fibroblasts

4: liver epithelial cells

5: thymic epithelial cells

6: intestinal stem cells

7: astrocytes

8: keratinocytes

9: bulge stem cells

Extended Data Fig. 6: L1 T $\mathrm{T}_{\mathrm{F}}$ subfamily promoter monomer methylation. a, $\mathrm{L} 1 \mathrm{~T}_{\mathrm{F}}$ monomer CpG methylation in MEFs, single-cell miPSC clones, and bulk miPSCs derived from primary cells. top: Assay design and primer locations with respect to L1 $\mathrm{T}_{\mathrm{F}}$ monomer structure. Orange strokes indicate $\mathrm{CpGs}$ covered by the assay. bottom: Histogram data represent the mean percentage methylation of 50 non-identical bisulfite converted sequences selected at random from each sample. A two-tailed $t$ test $(\mathrm{p}<0.001)$ was used to compare serum and $2 \mathrm{i}$ culture conditions for single-cell miPSC clones 1-4. $\mathbf{b}, \mathrm{L}_{1} \mathrm{~T}_{\mathrm{F}}$ methylation in four single-cell miPSC clones and parental MEFs. Each cartoon panel corresponds to an amplicon and displays 50 non-identical randomly selected sequences (black circle, methylated $\mathrm{CpG}$; white circle, unmethylated $\mathrm{CpG} ; \times$, mutated $\mathrm{CpG}$ ). Methylated $\mathrm{CpG}$ percentage is indicated underneath each cartoon. c, As per panel (b) except for bulk miPSC lines derived from animals A67 and A172. Note that this assay surveys $\mathrm{CpG}$ methylation for $\mathrm{T}_{\mathrm{F}}$ monomers genome-wide without retaining their position within individual L1 loci. 


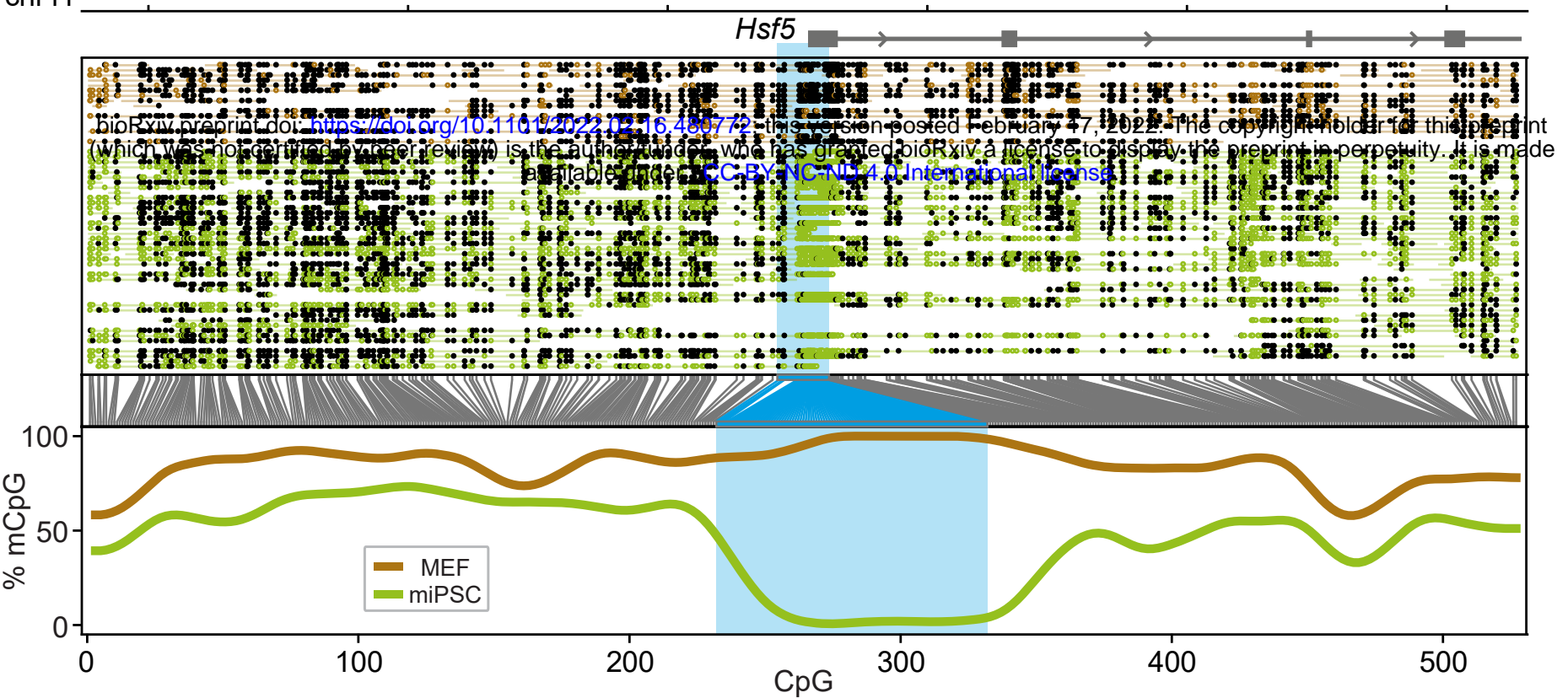

b c

IAP LTR $\square$

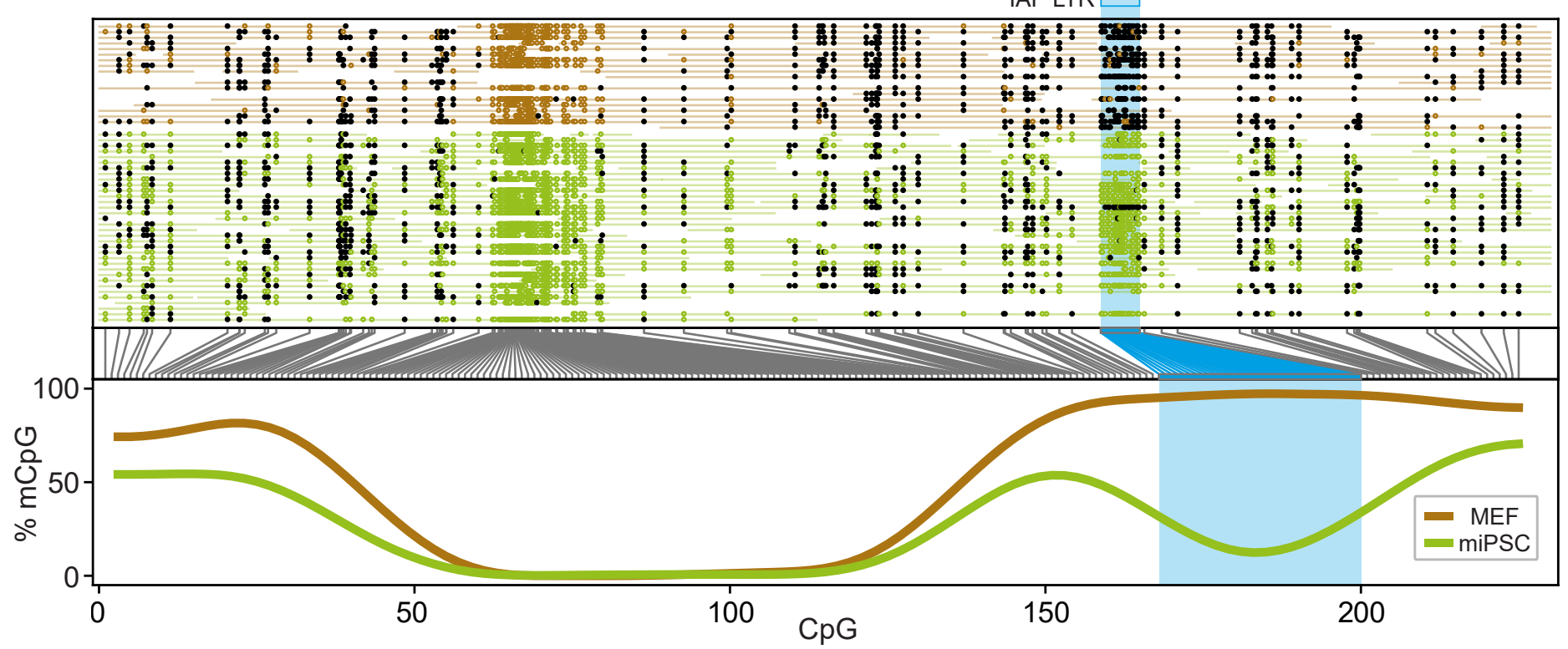

C $\operatorname{chr} 8 \underset{56,934,022}{ }$

$56,938,694$

$56,943,366$

$56,948,038$

$56,952,710$

$56,957,382$

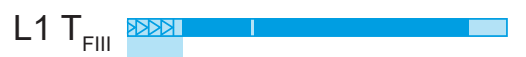

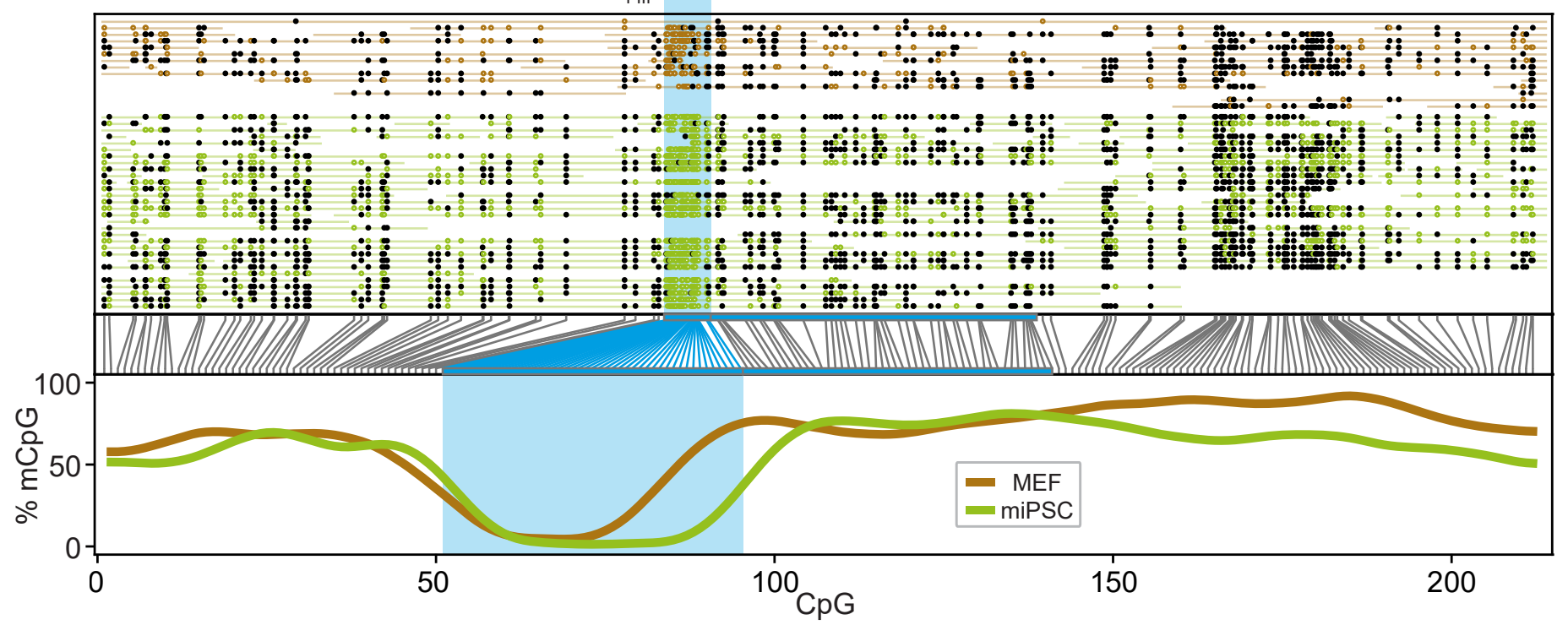

Extended Data Fig. 8: Examples of protein-coding gene and TE methylation, as surveyed by ONT sequencing. Methylation

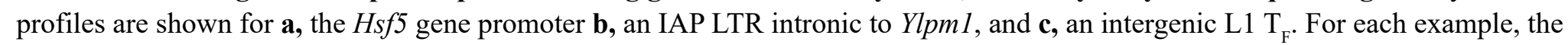
panels are arranged as per Fig. 3e. ONT data are shown for MEFs and a representative miPSC line untreated with lamivudine (CTRL 2). 